\title{
Melting properties of peptides and their solubility in water. Part 2: di- and tripeptides based on glycine, alanine, leucine, proline and serine
}

Hoang Tam Do ${ }^{\text {ał }}$, Yeong Zen Chua ${ }^{\text {bcc }}$, Jonas Habicht ${ }^{\mathrm{a}}$, Marcel Klinksiek ${ }^{\mathrm{a}}$, Sophia Volpert ${ }^{\mathrm{a}}$, Moritz Hallermann ${ }^{\mathrm{a}}$, Max Thome ${ }^{\mathrm{a}}$, Daniel Pabsch ${ }^{\mathrm{a}}$, Dzmitry Zaitsau ${ }^{\mathrm{c}, \mathrm{d}}$, Christoph Schick ${ }^{\mathrm{b}, \mathrm{c}, \mathrm{e}}$, Christoph Held ${ }^{a^{*}}$

${ }^{a}$ Laboratory of Thermodynamics, TU Dortmund University, Emil-Figge-Str. 70, 44227 Dortmund, Germany. Email: christoph.held@tu-dortmund.de

bInstitute of Physics, University of Rostock, Albert-Einstein-Str. 23-24, 18051 Rostock, Germany. E-mail: yeong.chua@uni-rostock.de

${ }^{c}$ Competence Centre CALOR, University of Rostock, Albert-Einstein-Str. 25, 18051 Rostock, Germany

Institute of Chemistry, University of Rostock, Dr-Lorenz-Weg 2, 18051 Rostock, Germany

${ }^{e}$ Chemical Institute A. M. Butlerov, Kazan Federal University, 18 Kremlyovskaya Street, Kazan 420008, Russian Federation

${ }^{\dagger}$ Electronic supplementary information (ESI) available. See DOI:

* Shared first authors.

* Corresponding author.

KEYWORDS peptides, melting temperature, melting enthalpy, heat capacity difference, aqueous solubility, $\mathrm{pH}$ measurement, PXRD, crystal structure, FSC, PC-SAFT

Supplementary Information 
Melting measurements with FSC. Experimental FSC melting properties measurements were carried out by using Flash DSC 1 (Mettler Toledo) with the calorimetric twin chip sensor UFS1. ${ }^{1,2}$ The experimental FSC study of the peptides was given in the previous works, where detailed experimental description of FSC method has been presented. ${ }^{3,4}$

All measurements were conducted under inert atmosphere of dry nitrogen (dew point lower than 150 $\mathrm{K}$ ) with a flow rate of $50 \mathrm{~mL} \cdot \mathrm{min}^{-1}$. The sensors were conditioned according to manufacturer's procedure and the temperature was calibrated with recommended calibration metals (indium, bismuth and tin).

The experimental FSC procedure consists of three measurement stages, as presented in the temperaturetime profile in Figure S1.

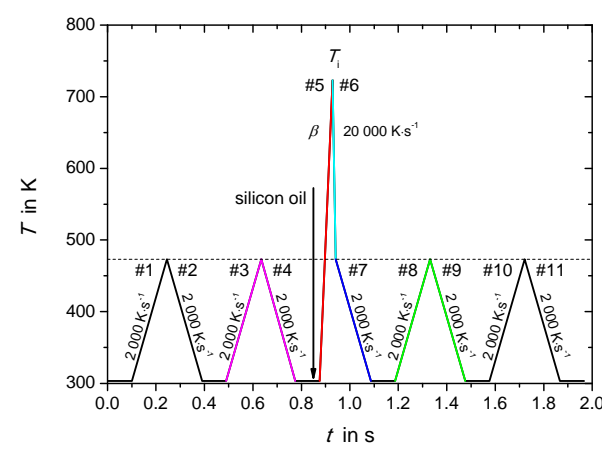

Figure S1 Temperature-time profile for determination of melting properties with fast scanning calorimetry. In heating step $\# 5$, the scanning rate, $\beta$, varied from 1000 $\mathrm{K} \cdot \mathrm{s}^{-1}$ to $20000 \mathrm{~K} \cdot \mathrm{s}^{-1}$.

The starting temperature is set to $303 \mathrm{~K}$ to reduce the measuring time, as starting temperature below $303 \mathrm{~K}$ requires a cooler and long system equilibration times.

For the first stage (\#1 - \#4), the temperature range from $303 \mathrm{~K}$ to $473 \mathrm{~K}$ and constant scanning rate $2000 \mathrm{~K} \cdot \mathrm{s}^{-1}$ were selected to assure the high reproducibility of the heating and cooling cycles. The reproducibility is an indirect proof indicating that sample mass loss due to sublimation or decomposition has not occurred, and that volatile impurities or water were absent. It is also indicating that the sample was measured in its anhydrous form. The sample mass (without silicon oil) is determined in this stage as $m_{0}=M_{i}\left[\mathrm{~g} \cdot \mathrm{mol}^{-1}\right]$. $C_{p 0 i}^{S}\left[J \cdot K^{-1}\right] / c_{p 0 i}^{S}\left[J \cdot m o l^{-1} \cdot K^{-1}\right]$, where $C_{p 0 i}^{S}[J \cdot$ $\left.K^{-1}\right]$ is the total heat capacity of the sample on the sensor from the first FSC stage and $c_{p 0 i}^{S}\left[J \cdot \mathrm{mol}^{-1}\right.$. $K^{-1}$ ] is specific heat capacity determined in DSC measurements (Pyris 1, PerkinElmer, USA). ${ }^{5-10}$

In the second stage, the melting properties were determined in heating step \#5. To improve the thermal contact between the sample and the sensor, silicon oil can be added to the sample before heating step \#5. All the samples used in FSC measurements were relatively small (less than $100 \mathrm{ng}$ ) and for such small samples, the surface-to-volume ratio is rather high, what leads to increase in mass loss due to sublimation or evaporation at higher temperature. This effect is especially prominent for small molecules like peptides. Therefore, silicon oil not only improves the thermal contact but additionally coats the sample surface and suppresses the mass loss of the sample due to sublimation or evaporation. The heating rates of step \#5 typically ranged from $2,000 \mathrm{~K} \cdot \mathrm{s}^{-1}$ to $10,000 \mathrm{~K} \cdot \mathrm{s}^{-1}$. Unfortunately, even with these approaches sublimation or evaporation of Pro-Ala cannot be suppressed fully enough. The melting and evaporation process were overlapping, what leads to an unsuccessful determination of melting properties.

In the heating step \#5 the shaded grey area in Figure 4(a) and Figure S7 in the temperature range of the melting peak was designated as the melting enthalpy, $\Delta H_{0 i}^{S L} \quad[\mathrm{~J}]$, while the onset of the melting peak is a scanning rate dependent melting temperature, $T_{0 i}^{S L}(ß)$. The specific melting enthalpy, $\Delta h_{0 i}^{S L}$, is defined as a ratio $\Delta h_{0 i}^{S L}=\Delta H_{0 i}^{S L} \cdot M / m_{0}$, where $\mathrm{M}$ is the molar mass of peptides and $m_{0}$ is the mass of the sample.

After heating step $\# 5$, the molten samples without silicon oil were quenched rapidly to retain the sample in the liquid state below the melting temperature without crystallization. During the heating and cooling cycles (\#8 - \#11) in third stage a step change in specific heat capacity corresponding to glass transition from amorphous solid of peptide to liquid (supercooled) state was observed. Due to complications in avoiding sublimation or evaporation mass loss of the samples at high temperatures in the current state of FSC technique, the glass transition of Pro-Ala cannot be determined. 


\section{Solubility determination with photometric method}

Table S1 Linear equation $A b s=a \cdot \widetilde{m}+b$ of the calibration curves. The coefficient of determination $\mathrm{R}^{2}$ is always above 0.98 . The dilution factor $\left(D F=\left(m_{\text {solution }}+m_{\text {water }}\right) \cdot m_{\text {water }}^{-1}\right)$ as well as the absorbance maximum at $\mathrm{n} / \mathrm{nm}$ is given. The calibration curves were applied to determine the peptide solubility with the photometric method.

\begin{tabular}{cccccc}
\hline Peptide & $\mathrm{a}$ & $\mathrm{b}$ & $R^{2}$ & $D F$ & $n / n m$ \\
\hline Gly-Gly & - & - & - & & \\
Gly-Ala & - & - & - & & \\
Ala-Gly & 6285.84 & 0.03 & 0.9889 & 14000 & 192 \\
Ala-Ala & 5859.88 & 0.04 & 0.9936 & 15000 & 190 \\
Gly-Ser & 6092.85 & 0.03 & 0.9946 & 15000 & 190 \\
Ser-Gly & 7468.21 & 0.01 & 0.9903 & 2500 & 190 \\
Ala-Ser & 5396.84 & 0.03 & 0.9820 & 30000 & 190 \\
Ser-Ala & 6849.9 & 0.04 & 0.9963 & 225 & 192 \\
Gly-Pro & 3222.41 & -0.02 & 0.9991 & 250000 & 199 \\
Pro-Gly & 6068.49 & 0.03 & 0.9977 & 10000 & 190 \\
Ala-Pro & 3878.75 & 0.01 & 0.9869 & 160000 & 203 \\
Pro-Ala & 6419.20 & 0.04 & 0.9988 & 2000 & 191 \\
Gly-Gly-Gly & 14005.79 & 0.02 & 0.9984 & 7000 & 190 \\
Gly-Gly-Ala & 13508.57 & 0.03 & 0.9978 & 5000 & 190 \\
Gly-Ala-Gly & 13643.21 & 0.05 & 0.9881 & 4000 & 190 \\
Ala-Gly-Ala & 7842.75 & 0.05 & 0.9945 & 60000 & 190 \\
Ala-Ala-Ala & 13379.51 & 0.02 & 0.9975 & 15000 & 190 \\
Leu-Gly-Gly & 11693.21 & 0.03 & 0.9946 & 20000 & 190 \\
Gly-Leu-Gly & 13440.66 & 0.03 & 0.9974 & 4000 & 190 \\
Gly-Gly-Leu & 11729.85 & 0.03 & 0.9957 & 15000 & 190 \\
Pro-Gly-Gly & 5722.54 & 0.04 & 0.9943 & 80000 & 190 \\
Gly-Ala-Leu & 14569.48 & 0.001 & 0.9828 & 900 & 190 \\
\hline
\end{tabular}




\section{Henderson Hasselbalch equation, $\mathrm{pk}_{\mathrm{a}}$ values and $\mathrm{pH}$ measurements}

Table S2 Dissociations schemes together with the corresponding Henderson Hasselbalch equation and the components. The consideration of the way of dissociation is adapted from A. Avdeef, $2007^{11}$

Dissociation

$\mathrm{H}_{2} A^{+} \rightleftharpoons H A^{ \pm} \rightleftharpoons A^{-}$

$\mathrm{H}_{3} A^{+} \rightleftharpoons \mathrm{H}_{2} A^{ \pm} \rightleftharpoons H A^{-}$

$\rightleftharpoons A^{2-}$

$10^{p K_{a 1}-p H}+10^{p H-p K_{a 2}}$

$$
+10^{2 p H-p K_{a 3}-p K_{a 2}}
$$

$$
\begin{aligned}
& 10^{p K_{a 1}-p H}+10^{p H-p K_{a 2}} \\
& +10^{2 p H-p K_{a 3}-p K_{a 2}} \\
& +10^{3 p H-p K_{a 4}-p K_{a 3}-p K_{a 2}}
\end{aligned}
$$

\section{Peptide}

Gly-Pro,

Ala-Pro

Gly-Gly, Gly-Ala, Ala-Gly, Ala-Ala, Pro-Gly, Pro-Ala, Leu-Gly-Gly

Gly-Ser, Ser-Gly, Ala-Ser, Ser-Ala, Gly-Gly-Gly, Gly-Gly-Ala, Gly-Ala-Gly, Ala-GlyAla, Ala-Ala-Ala, Gly-Gly-Leu, Gly-Leu-Gly, GlyAla-Leu, Pro-Gly-Gly

$$
x_{H A, t o t a l}^{L}=x_{H A}^{L} \cdot\left[1+10^{p K_{a 1}-p H}+10^{p H-p K_{a 2}}+10^{2 p H-p K a 3-p K a 2}+10^{3 p H-p K a 4-p K a 3-p K a 2}\right]
$$


Table S3 pK $\mathrm{p}_{\mathrm{a}}$ values for the dipeptides studied in the current work evaluated with Chemicalize ${ }^{\circledR}$ platform.

\begin{tabular}{|c|c|c|c|c|}
\hline Peptide & $p K_{a, 1}$ & $p K_{a, 2}$ & $p K_{a, 3}$ & $p K_{a, 4}$ \\
\hline Gly & 2.31 & 9.24 & - & - \\
\hline Ala & 2.47 & 9.48 & - & - \\
\hline Val & 2.72 & 9.60 & - & - \\
\hline Leu & 2.79 & 9.52 & - & - \\
\hline Ile & 2.32 & 9.76 & - & - \\
\hline Pro & 1.94 & 11.33 & - & - \\
\hline Ser & 2.03 & 8.93 & 15.17 & - \\
\hline Thr & 2.21 & 9.00 & 14.95 & - \\
\hline Asp & 1.70 & 5.11 & 9.61 & \\
\hline Asn & 2.00 & 8.43 & - & - \\
\hline Glu & 1.88 & 4.27 & 9.54 & \\
\hline Gln & -1.63 & 2.15 & 9.31 & - \\
\hline Arg & -0.54 & 2.41 & 9.12 & 12.41 \\
\hline His & 1.85 & 6.61 & 9.44 & 12.94 \\
\hline Lys & 2.74 & 9.44 & 10.29 & \\
\hline Phe & 2.47 & 9.45 & - & - \\
\hline Tyr & 2.20 & 9.11 & 10.13 & \\
\hline Trp & 2.43 & 9.44 & - & - \\
\hline Cys & 2.35 & 9.05 & 10.17 & \\
\hline Met & 2.53 & 9.50 & - & - \\
\hline Gly-Gly & 3.52 & 8.14 & 15.32 & - \\
\hline Gly-Ala & 3.61 & 8.14 & 13.82 & - \\
\hline Ala-Gly & 3.66 & 8.39 & 14.32 & - \\
\hline Ala-Ala & 3.73 & 8.39 & 13.82 & - \\
\hline Gly-Ser & 3.36 & 8.14 & 12.66 & 15.11 \\
\hline Ser-Gly & 3.44 & 7.85 & 13.92 & 15.15 \\
\hline Ala-Ser & 3.48 & 8.39 & 12.72 & 15.11 \\
\hline Ser-Ala & 3.51 & 7.85 & 13.50 & 15.13 \\
\hline Gly-Pro & 3.61 & 8.13 & - & - \\
\hline Pro-Gly & 3.66 & 9.81 & 14.29 & - \\
\hline Ala-Pro & 3.71 & 8.38 & - & - \\
\hline Pro-Ala & 3.71 & 9.81 & 13.79 & - \\
\hline Gly-Gly-Gly & 3.43 & 7.84 & 12.54 & 15.84 \\
\hline Gly-Gly-Ala & 3.49 & 7.84 & 12.60 & 14.43 \\
\hline Gly-Ala-Gly & 3.53 & 7.84 & 12.43 & 14.61 \\
\hline Ala-Gly-Ala & 3.58 & 8.09 & 12.64 & 14.39 \\
\hline Ala-Ala-Ala & 3.65 & 8.09 & 12.53 & 14.19 \\
\hline Gly-Gly-Leu & 3.72 & 7.82 & 12.82 & 14.16 \\
\hline Gly-Leu-Gly & 3.77 & 7.84 & 12.61 & 14.81 \\
\hline Leu-Gly-Gly & 3.39 & 8.13 & 12.82 & - \\
\hline Pro-Gly-Gly & 3.26 & 9.50 & 12.60 & 15.94 \\
\hline Gly-Ala-Leu & 3.78 & 7.84 & 12.68 & 14.24 \\
\hline
\end{tabular}


Table S4 pH values of the unbuffered saturated peptide - water solutions at different temperatures

\begin{tabular}{|c|c|c|c|c|c|c|c|}
\hline Peptide $\backslash \mathrm{T} / \mathrm{K}$ & 288.15 & 293.15 & 298.15 & 303.15 & 308.15 & 313.15 & 323.15 \\
\hline Gly-Gly & - & $5.90 \pm 0.01$ & $5.85 \pm 0.01$ & $\begin{array}{c}5.86 \pm 0.01 \\
\text { (at } 301.15 \mathrm{~K} \text { ) }\end{array}$ & $5.85 \pm 0.02$ & $5.82 \pm 0.01$ & - \\
\hline Gly-Ala & $6.15 \pm 0.01$ & $6.17 \pm 0.01$ & $6.08 \pm 0.02$ & $6.09 \pm 0.01$ & - & $5.96 \pm 0.05$ & $5.88 \pm 0.03$ \\
\hline Ala-Gly & $5.91 \pm 0.01$ & $5.95 \pm 0.01$ & $5.85 \pm 0.01$ & $5.85 \pm 0.01$ & - & $5.80 \pm 0.04$ & $5.60 \pm 0.02$ \\
\hline Ala-Ala & - & $5.78 \pm 0.02$ & $5.77 \pm 0.02$ & $5.79 \pm 0.01$ & - & $5.71 \pm 0.01$ & $5.65 \pm 0.01$ \\
\hline Gly-Ser & - & $5.67 \pm 0.01$ & $5.73 \pm 0.03$ & $5.73 \pm 0.01$ & - & $5.70 \pm 0.01$ & $5.62 \pm 0.01$ \\
\hline Ser-Gly & $5.25 \pm 0.01$ & $5.32 \pm 0.01$ & $5.14 \pm 0.01$ & $4.95 \pm 0.01$ & $4.73 \pm 0.02$ & - & - \\
\hline Ala-Ser & - & $5.70 \pm 0.02$ & $5.70 \pm 0.01$ & $5.67 \pm 0.05$ & - & $5.63 \pm 0.01$ & $5.60 \pm 0.01$ \\
\hline Ser-Ala & $5.22 \pm 0.01$ & $5.27 \pm 0.03$ & $5.09 \pm 0.01$ & $5.13 \pm 0.07$ & $5.03 \pm 0.01$ & - & - \\
\hline Gly-Pro & $6.93 \pm 0.03$ & $6.83 \pm 0.02$ & $6.88 \pm 0.01$ & $6.58 \pm 0.01$ & - & $6.71 \pm 0.02$ & $6.52 \pm 0.02$ \\
\hline Pro-Gly & - & $5.98 \pm 0.01$ & $6.02 \pm 0.01$ & $6.00 \pm 0.01$ & - & $5.95 \pm 0.01$ & $5.94 \pm 0.01$ \\
\hline Ala-Pro & - & $6.50 \pm 0.01$ & $6.40 \pm 0.01$ & $6.45 \pm 0.02$ & - & $6.41 \pm 0.02$ & $6.50 \pm 0.03$ \\
\hline Pro-Ala & $6.07 \pm 0.01$ & $6.10 \pm 0.02$ & $6.04 \pm 0.01$ & $5.99 \pm 0.02$ & $5.99 \pm 0.02$ & - & - \\
\hline Gly-Gly-Gly & - & $5.66 \pm 0.01$ & $5.69 \pm 0.02$ & $5.77 \pm 0.01$ & - & $5.69 \pm 0.02$ & $5.52 \pm 0.01$ \\
\hline Gly-Gly-Ala & - & $5.43 \pm 0.01$ & $5.51 \pm 0.03$ & $5.80 \pm 0.11$ & - & $6.15 \pm 0.18$ & $5.99 \pm 0.18$ \\
\hline Gly-Ala-Gly & - & $4.66 \pm 0.03$ & $4.64 \pm 0.01$ & $4.57 \pm 0.02$ & - & $4.35 \pm 0.03$ & $4.52 \pm 0.04$ \\
\hline Ala-Gly-Ala & $5.95 \pm 0.01$ & $5.91 \pm 0.02$ & $5.91 \pm 0.02$ & $5.87 \pm 0.01$ & $5.84 \pm 0.01$ & - & - \\
\hline Ala-Ala-Ala & - & $5.51 \pm 0.20$ & $5.51 \pm 0.29$ & $5.96 \pm 0.49$ & $5.96 \pm 0.49$ & - & - \\
\hline Gly-Gly-Leu & $5.74 \pm 0.03$ & $5.74 \pm 0.02$ & $5.75 \pm 0.01$ & $5.71 \pm 0.01$ & $5.70 \pm 0.01$ & - & - \\
\hline Gly-Leu-Gly & - & $5.35 \pm 0.01$ & $5.37 \pm 0.01$ & $5.48 \pm 0.15$ & - & $5.39 \pm 0.14$ & $5.24 \pm 0.03$ \\
\hline Leu-Gly-Gly & - & $5.43 \pm 0.01$ & $5.38 \pm 0.01$ & $5.34 \pm 0.02$ & - & $5.23 \pm 0.01$ & $5.21 \pm 0.01$ \\
\hline Pro-Gly-Gly & $6.52 \pm 0.01$ & $6.58 \pm 0.01$ & $6.48 \pm 0.01$ & $6.48 \pm 0.02$ & $6.48 \pm 0.01$ & - & - \\
\hline Gly-Ala-Leu & - & $5.81 \pm 0.01$ & $5.89 \pm 0.01$ & $6.04 \pm 0.13$ & - & $6.08 \pm 0.20$ & $5.81 \pm 0.04$ \\
\hline
\end{tabular}


Species contribution of all peptides used in this work
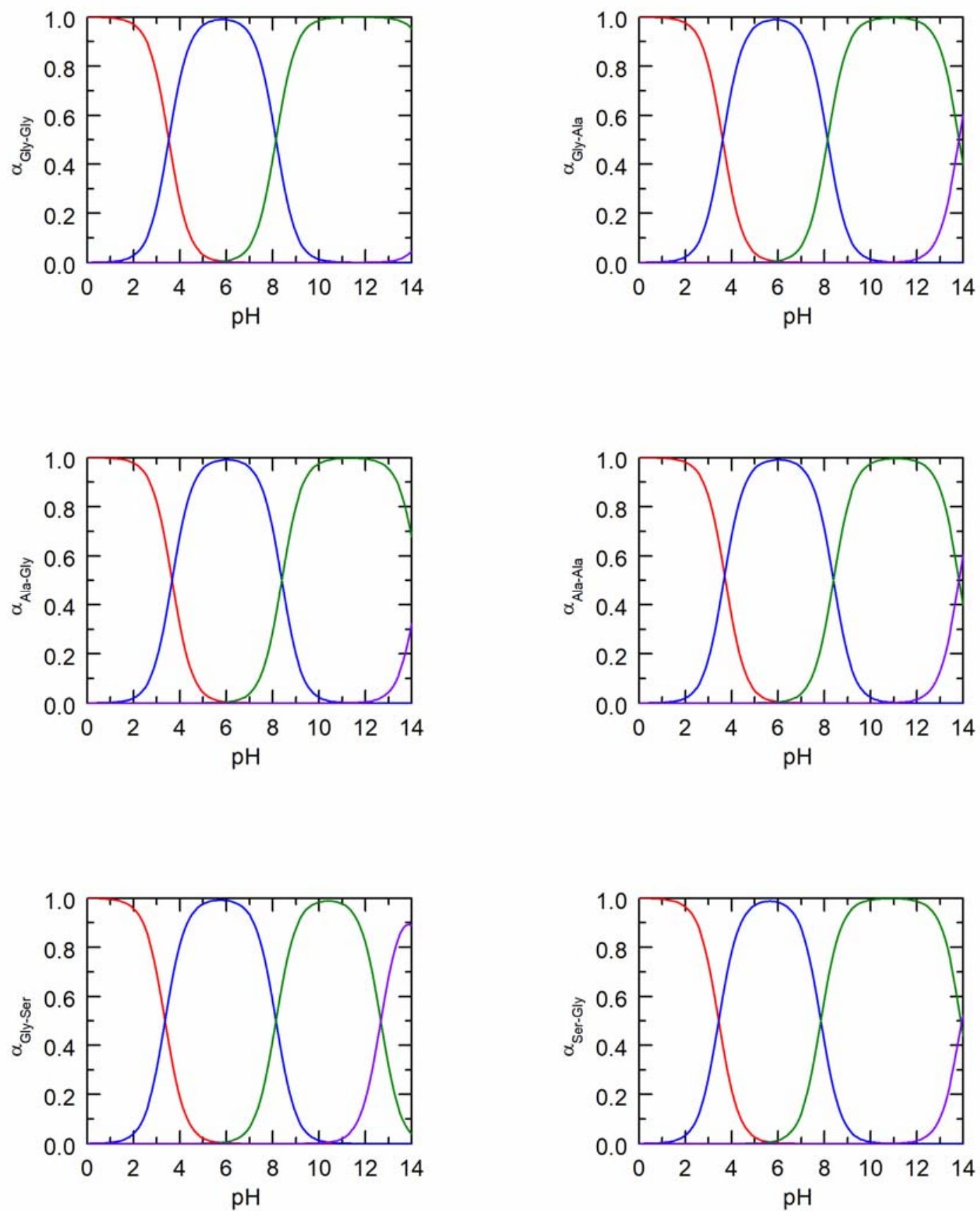

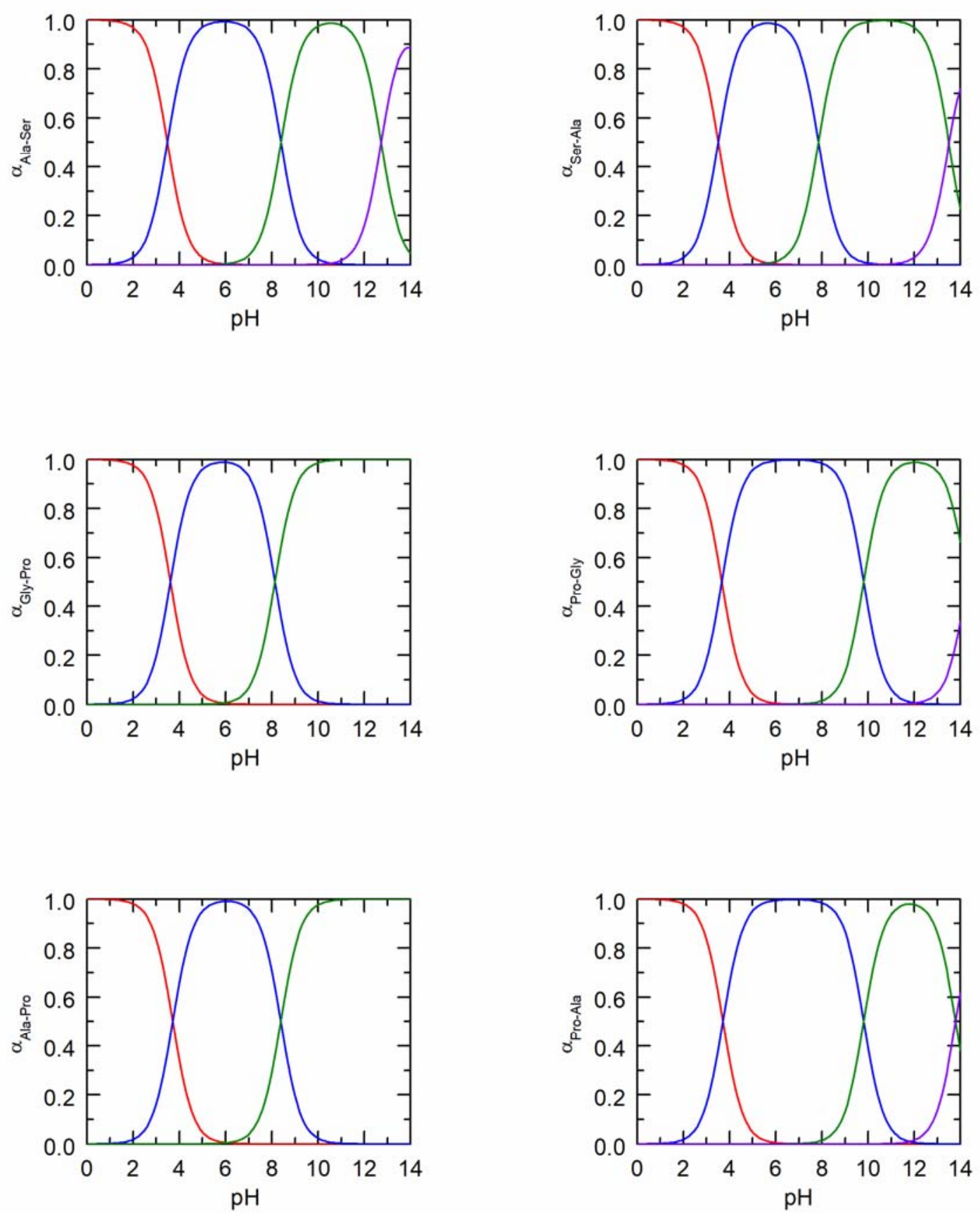

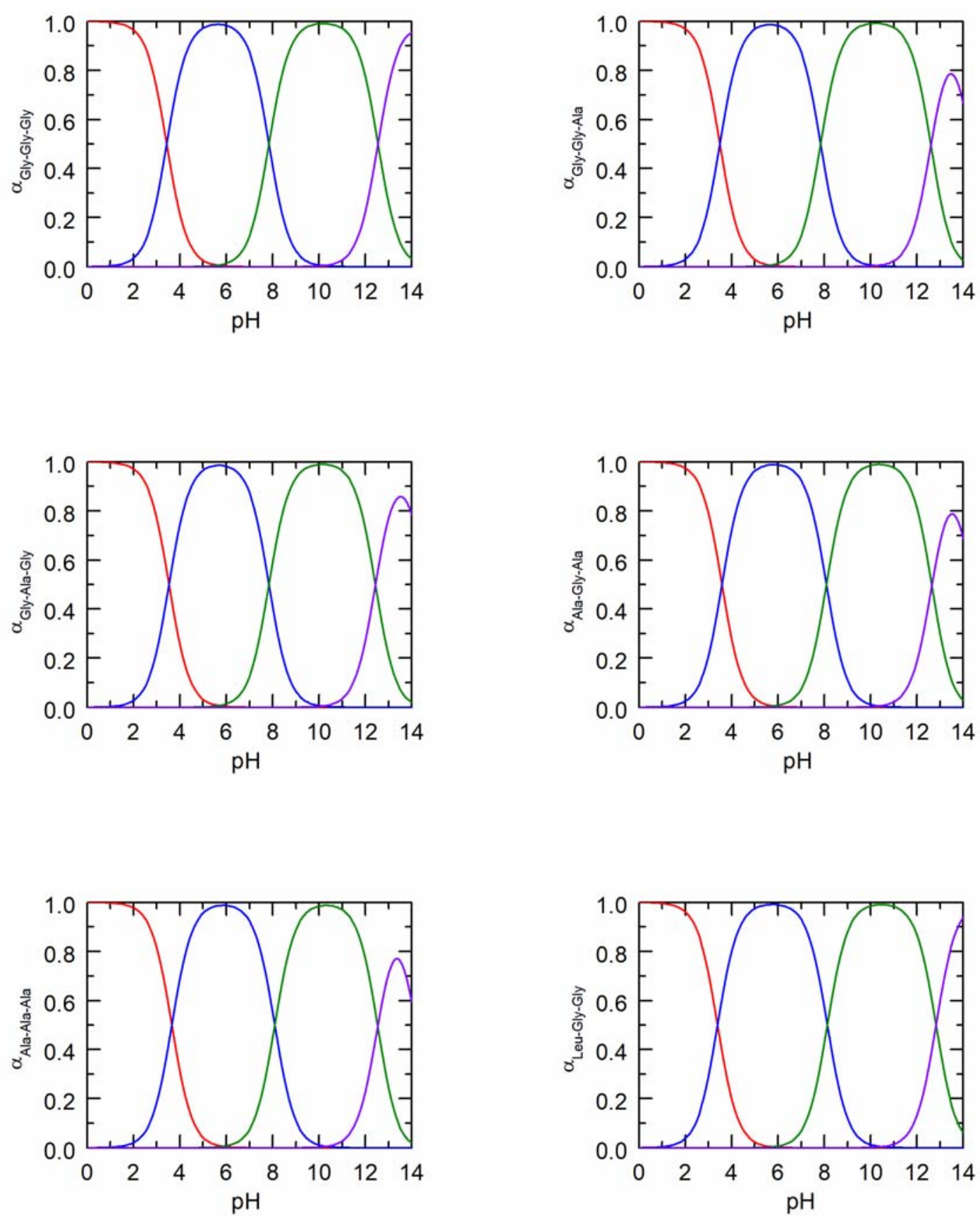

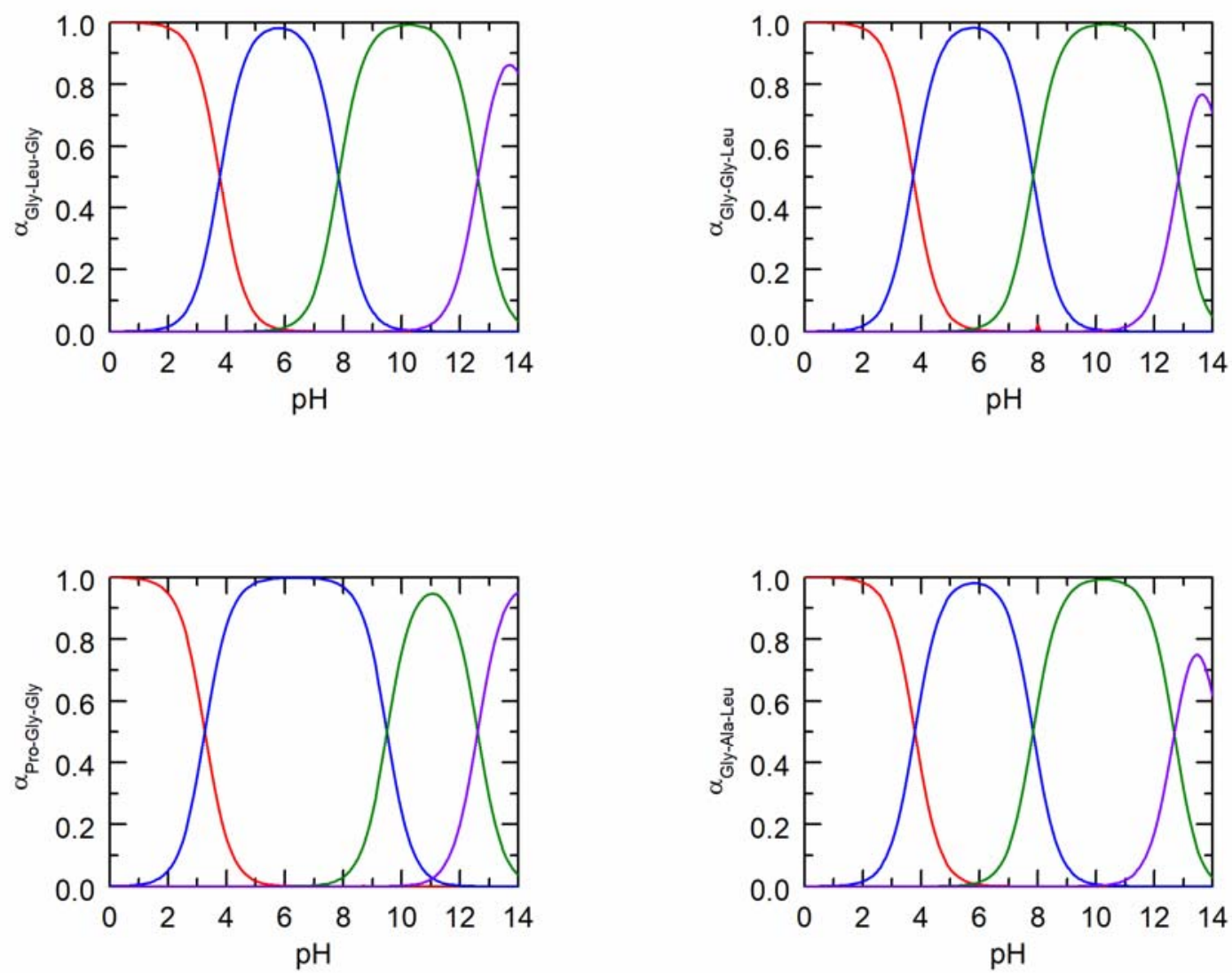

Figure S2 The $\mathrm{pH}$ distribution of peptides species in aqueous solution at $\mathrm{T}=298.15 \mathrm{~K}$ and $\mathrm{p}=1$ atm. Lines represent the contribution of species: positive net charged specie (red), zero net charge specie (blue), one negative net charged specie (green), species with double negative charge (violet). 
PC-SAFT. The activity coefficient in eq. (1) is the ratio of the fugacity coefficient in its saturated solution with mole fraction $\varphi_{i}^{L}$ and pure-component state $\varphi_{0 i}^{L}$. In the PC-SAFT equation of state the fugacity coefficient is expressed as

$$
\ln \varphi_{\mathrm{i}}=\frac{\mu_{\mathrm{i}}^{\mathrm{res}}}{\mathrm{RT}}-\ln (\mathrm{Z})
$$

where $\mu_{i}^{r e s}$ represents the residual chemical potential and $Z$ the real gas factor. The residual Helmholtz energy $a^{\text {res }}$ is required for the calculation of $\mu_{i}^{\text {res }}$ and $Z$ which is expressed in this work as

$$
a^{\text {res }}=a^{\text {hc }}+a^{\text {disp }}+a^{\text {assoc }}
$$

where $a^{h c}, a^{\text {disp }}$ and $a^{a s s o c}$ are the Helmholtz energy contributions hard chain repulsion, dispersion energy and association interactions respectively. These contributions have been implemented already in the original PC-SAFT from Gross and Sadowski ${ }^{12}$. For mixed solutions the conventional Berthelot-Lorentz ${ }^{13}$ combining rules were applied to describe the interactions between two components $i$ and $j$ (e.g. water and peptide)

$$
\begin{gathered}
\sigma_{i j}=\frac{1}{2}\left(\sigma_{i}+\sigma_{j}\right) \\
u_{i j}=\sqrt{u_{i} u_{j}}\left(1-k_{i j}\right)
\end{gathered}
$$

where $k_{i j}$ is the binary interaction parameter to describe deviations of the dispersion-energy parameters of two components $i$ and $j$.

Two pure-component parameters determine the associating interactions between the association site $A_{i}$ and $\mathrm{B}_{\mathrm{i}}$. The contributions are given with the association energy $\varepsilon^{A i B j} / \mathrm{k}$ and the association volume $\kappa^{A i B j}$. The combining rules for cross-association were suggested by Wolbach and Sandler without further interaction parameters $^{14}$

$$
\varepsilon^{A i B j}=\frac{1}{2}\left(\varepsilon^{A i B i}+\varepsilon^{A j B j}\right)
$$

$$
\kappa^{A i B j}=\sqrt{\kappa^{A i B i} \kappa^{A j B j}}\left(\frac{\sqrt{\sigma_{i i} \sigma_{j j}}}{1 / 2\left(\sigma_{i i}+\sigma_{j j}\right)}\right)^{3}
$$

In this work two different units were used. The peptide concentration are calculated based on molar fractions. For presentation reason the molar fraction was calculated to weight fraction and molality using eq. (S8) and (S9)

$$
\begin{gathered}
\widetilde{m_{2}}=\frac{x_{2}}{M_{1}\left(1-x_{2}\right)} \\
w_{2}=\frac{M_{2} \cdot x_{2}}{M_{1} \cdot x_{1}+M_{2} \cdot x_{2}}
\end{gathered}
$$


PC-SAFT osmotic coefficient, density, activity coefficient and solubility modeling results
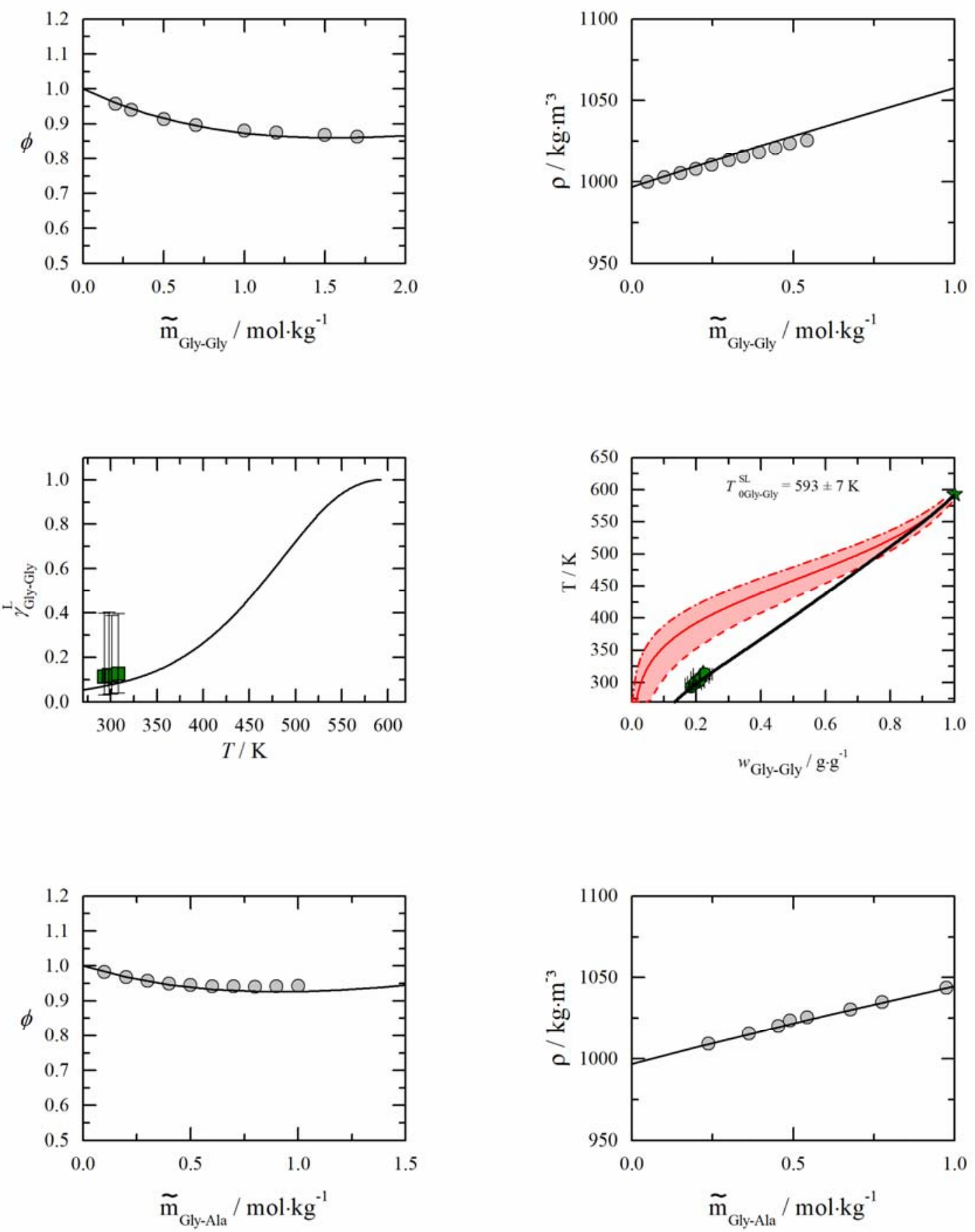

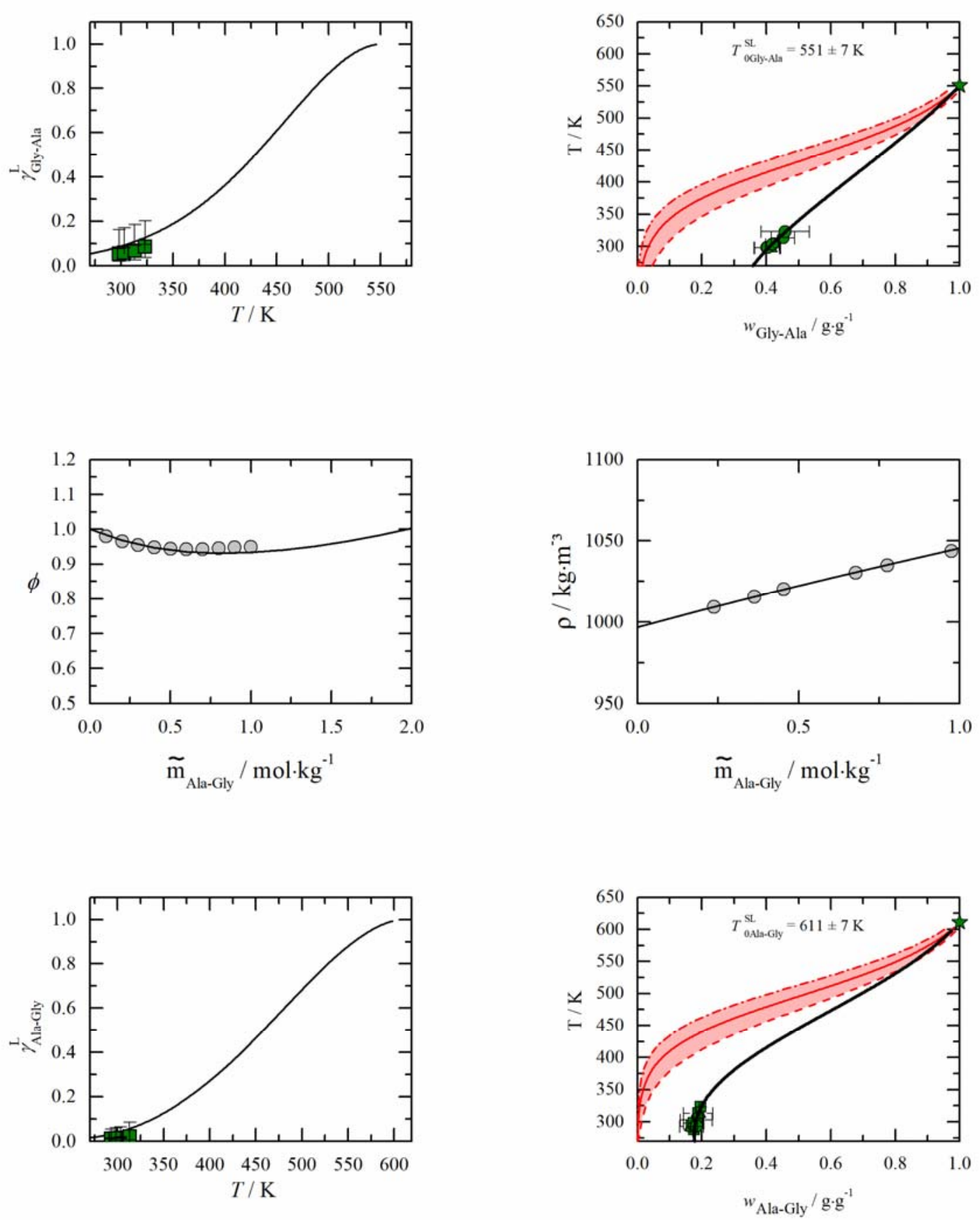

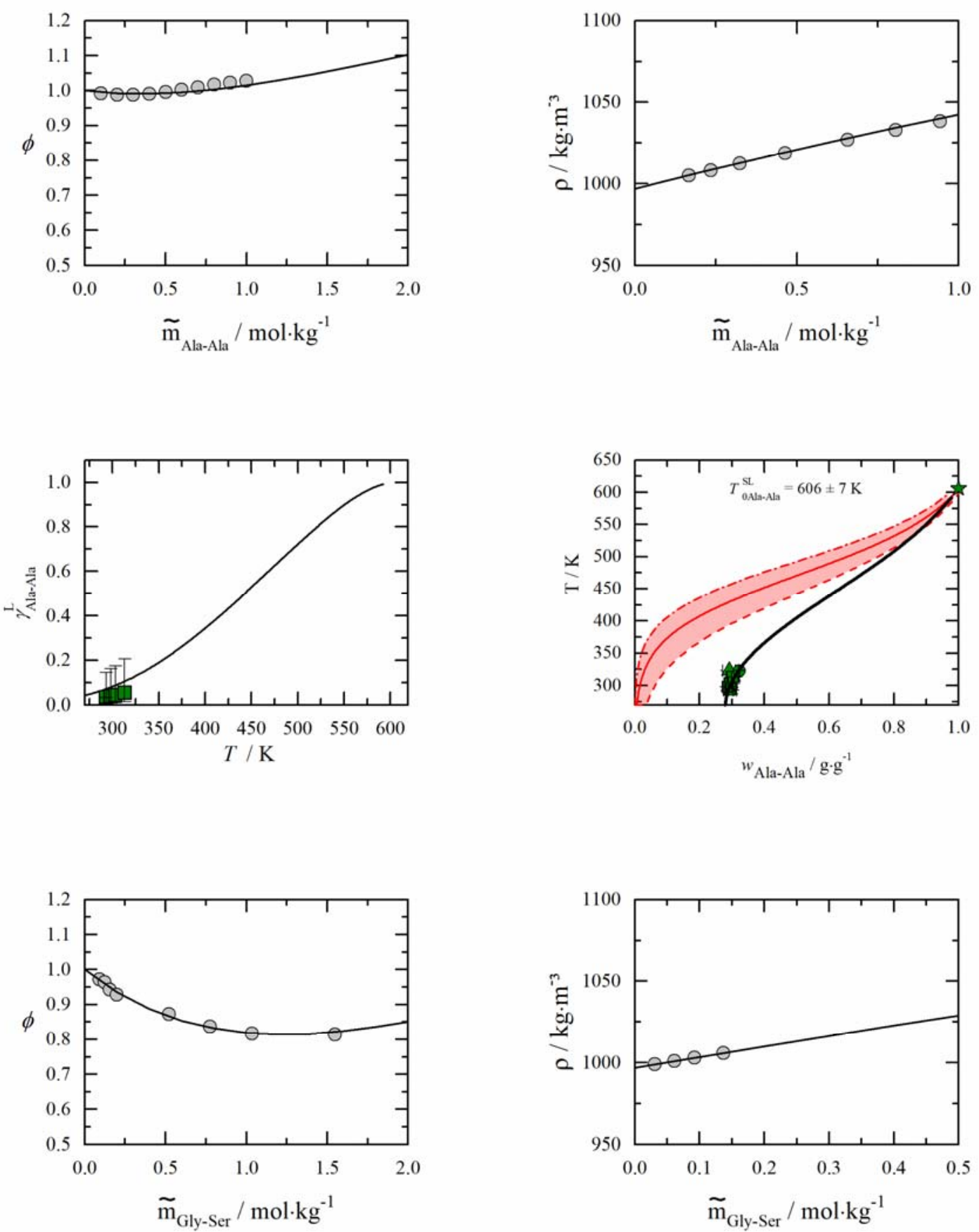

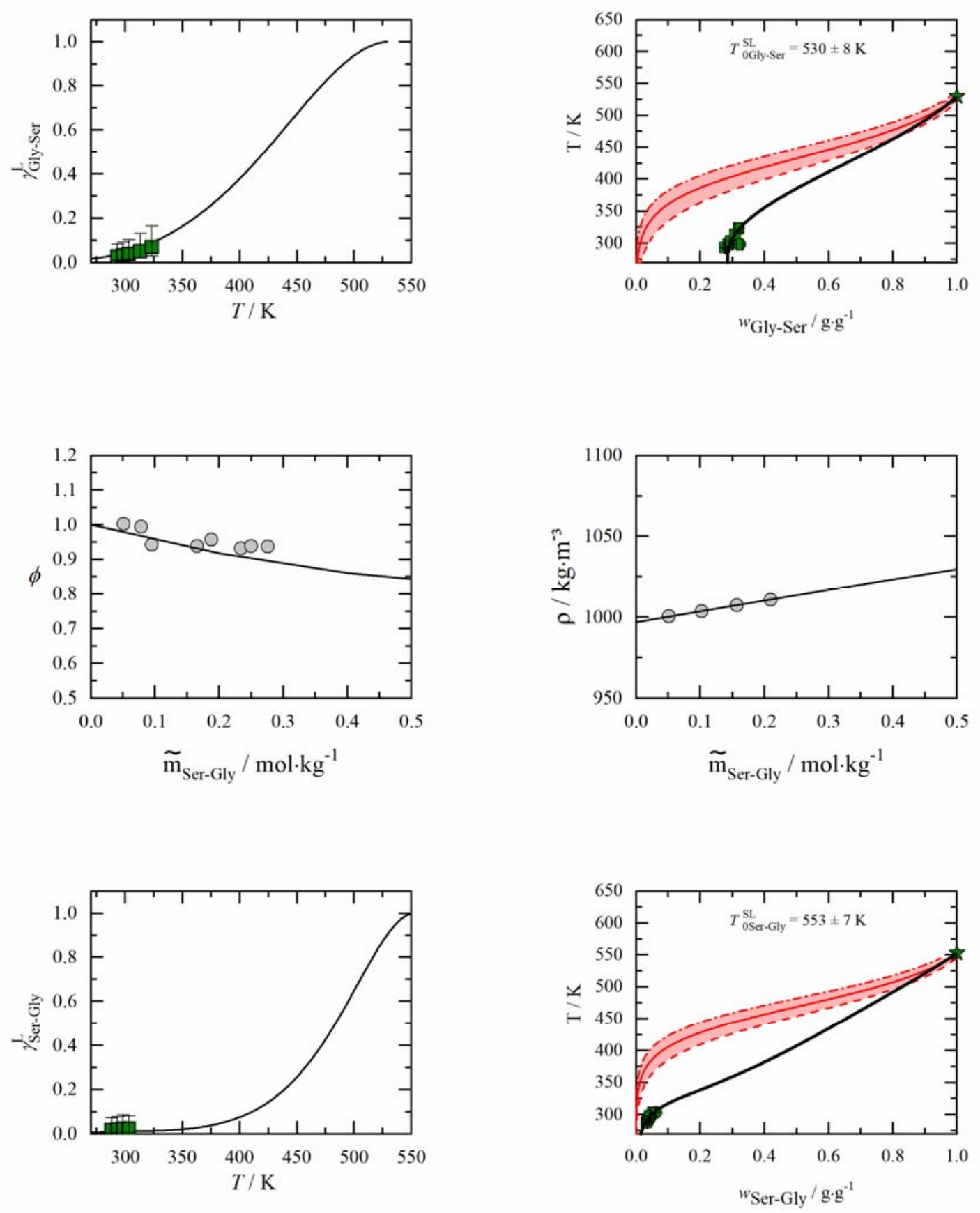

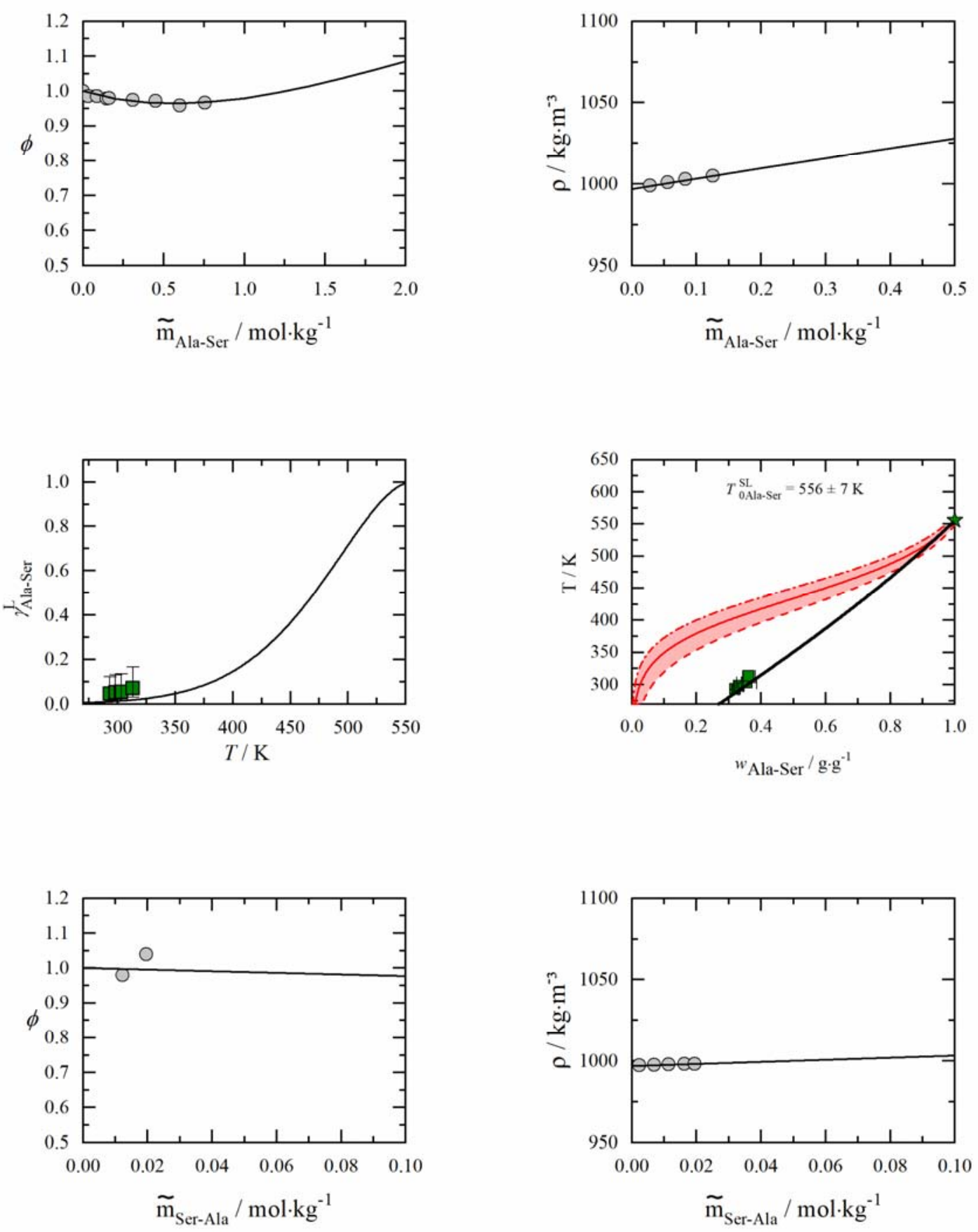

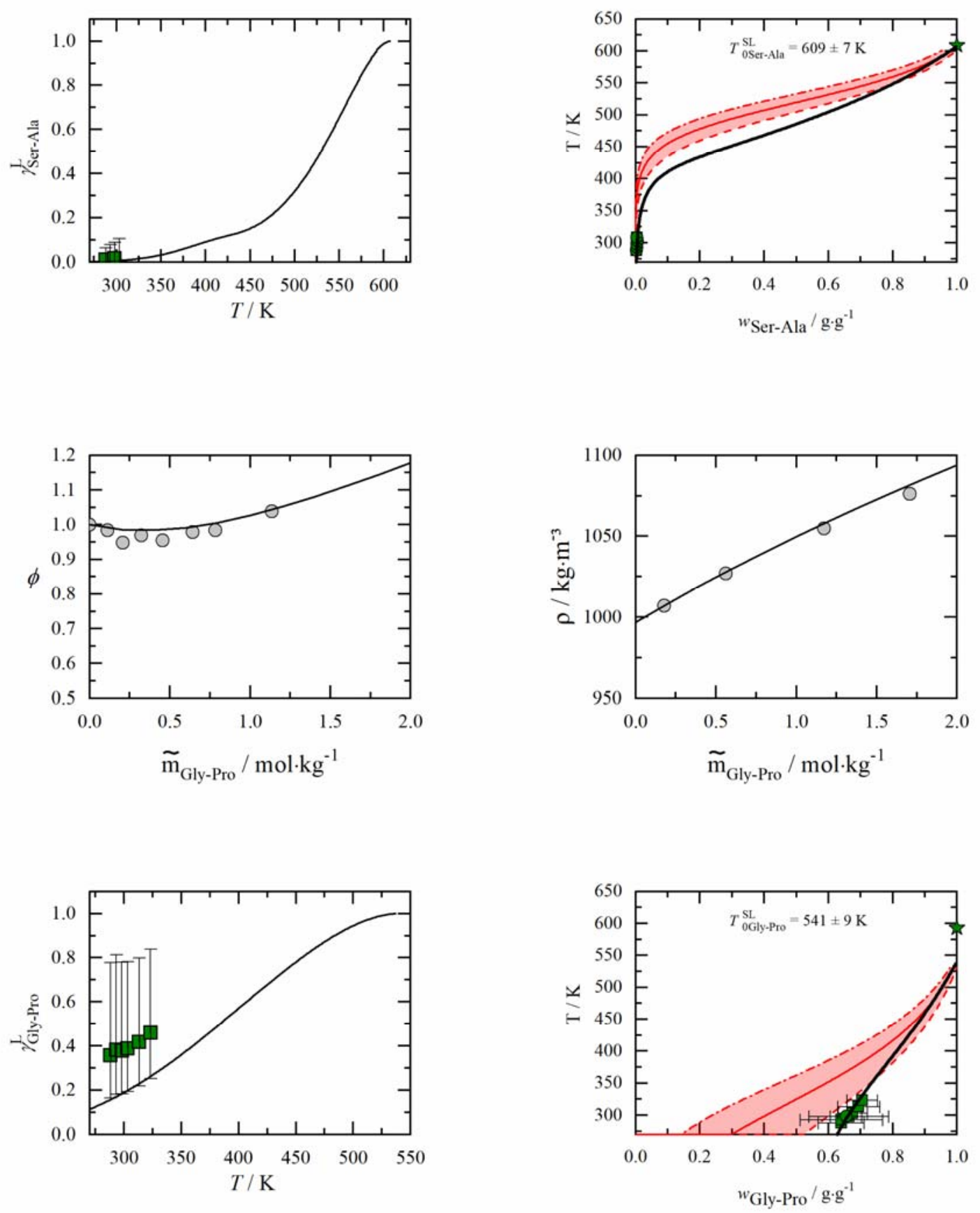

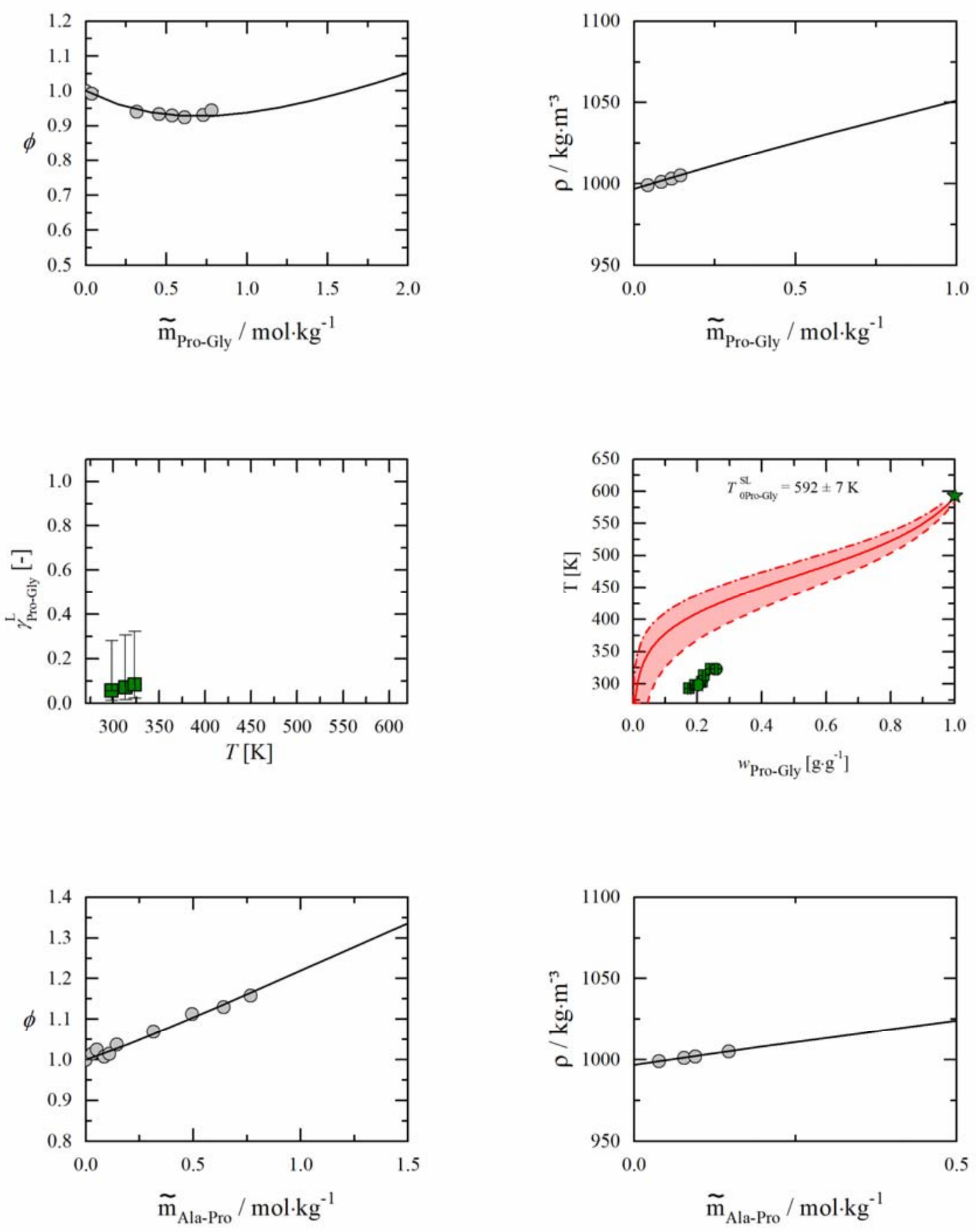

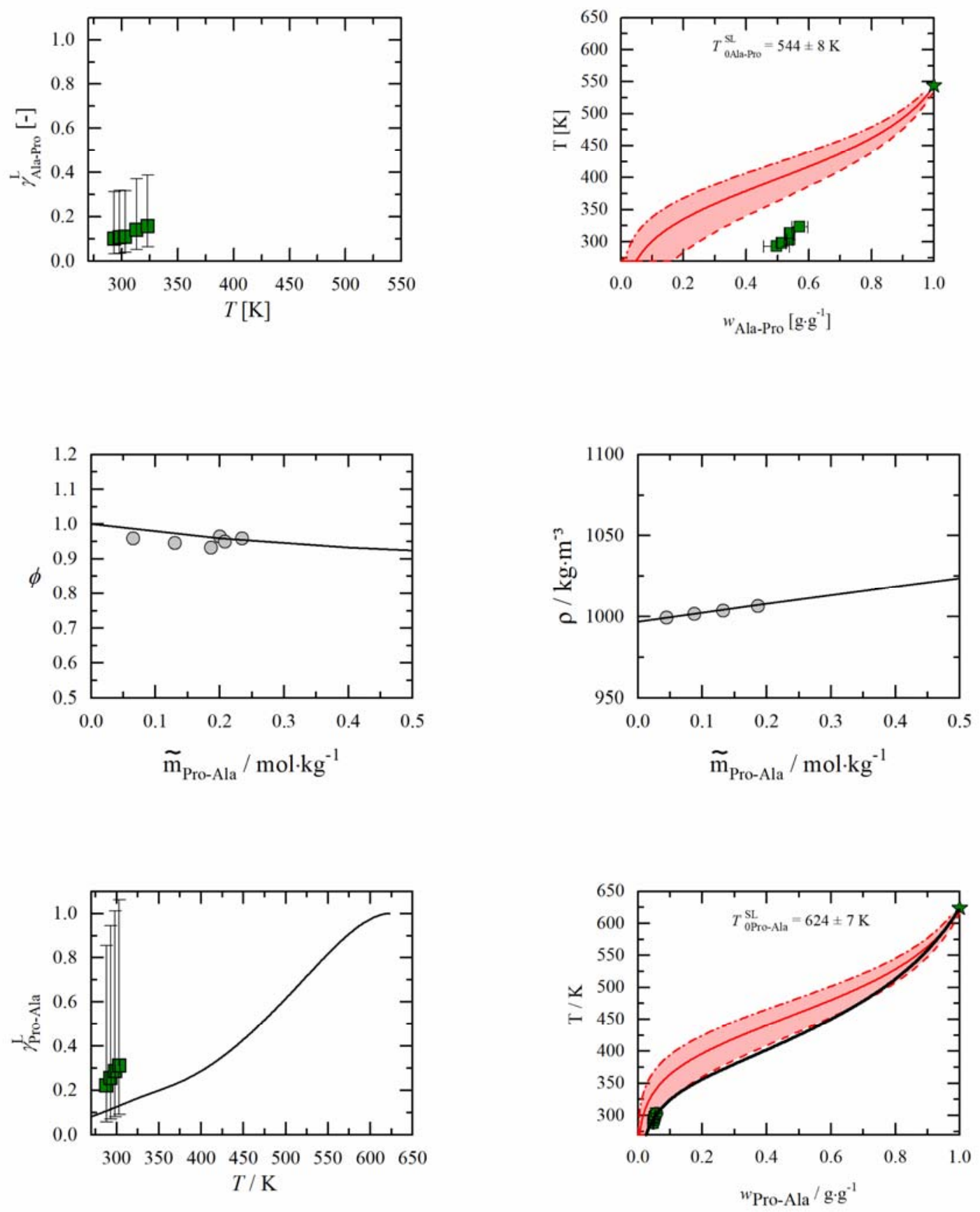

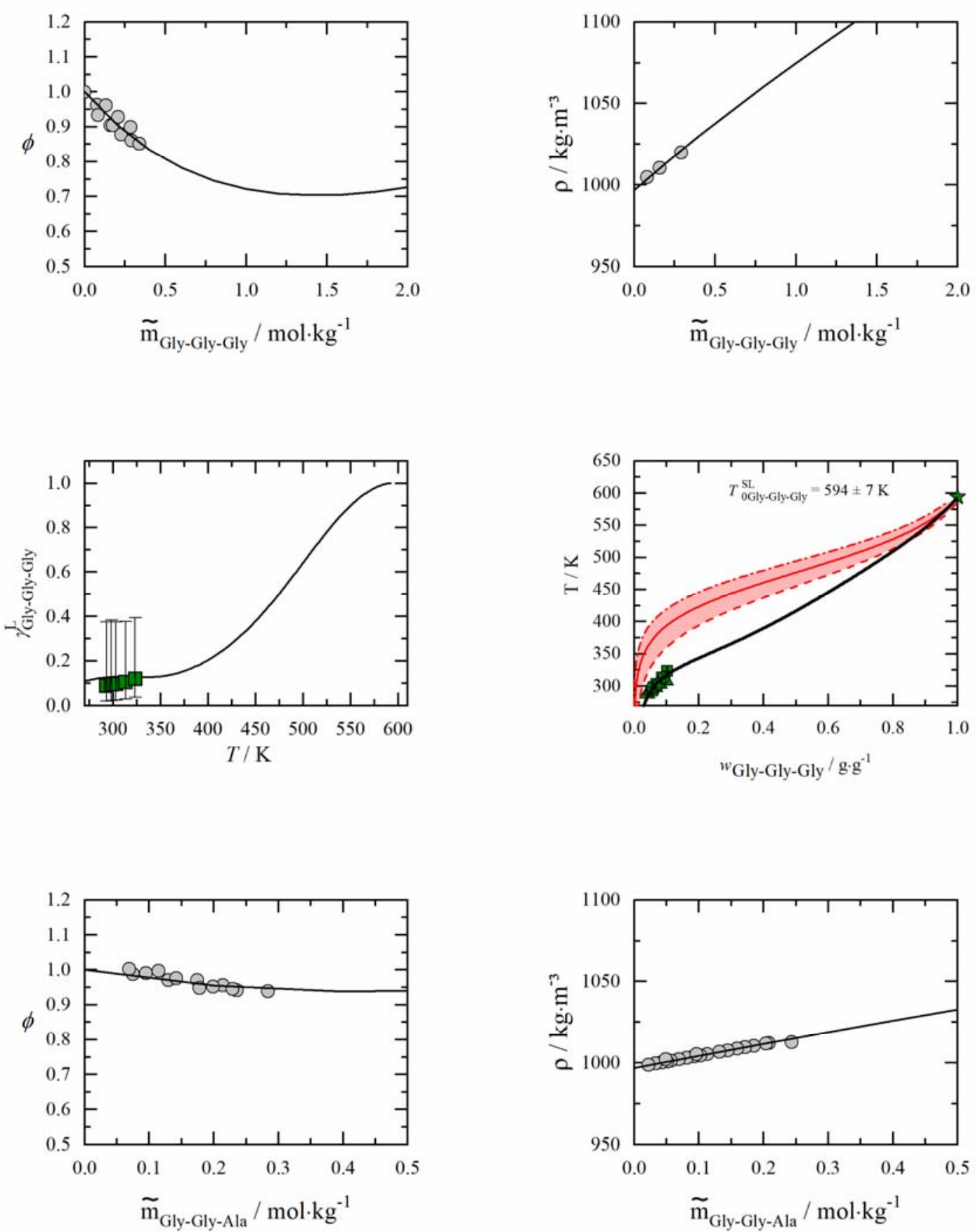

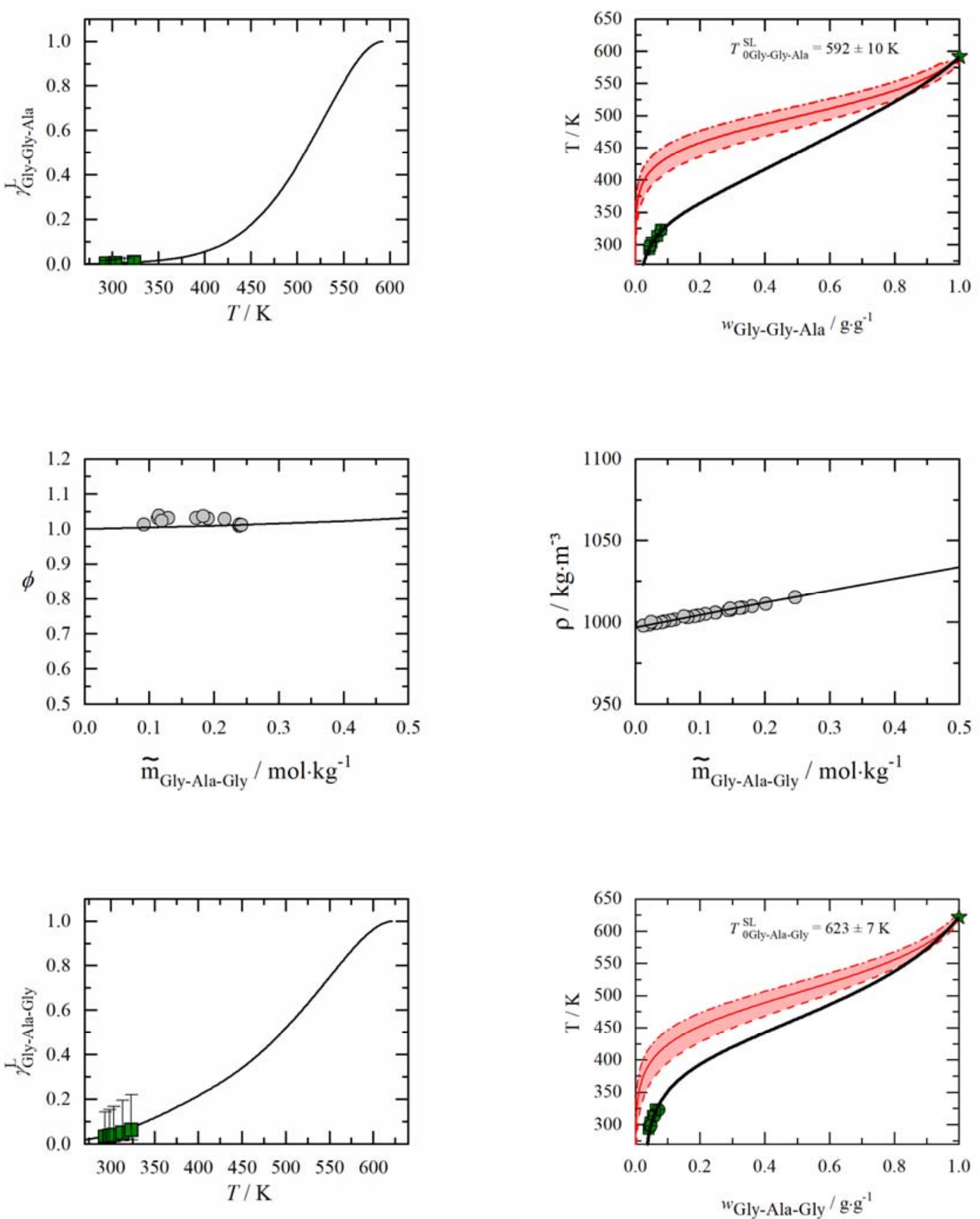

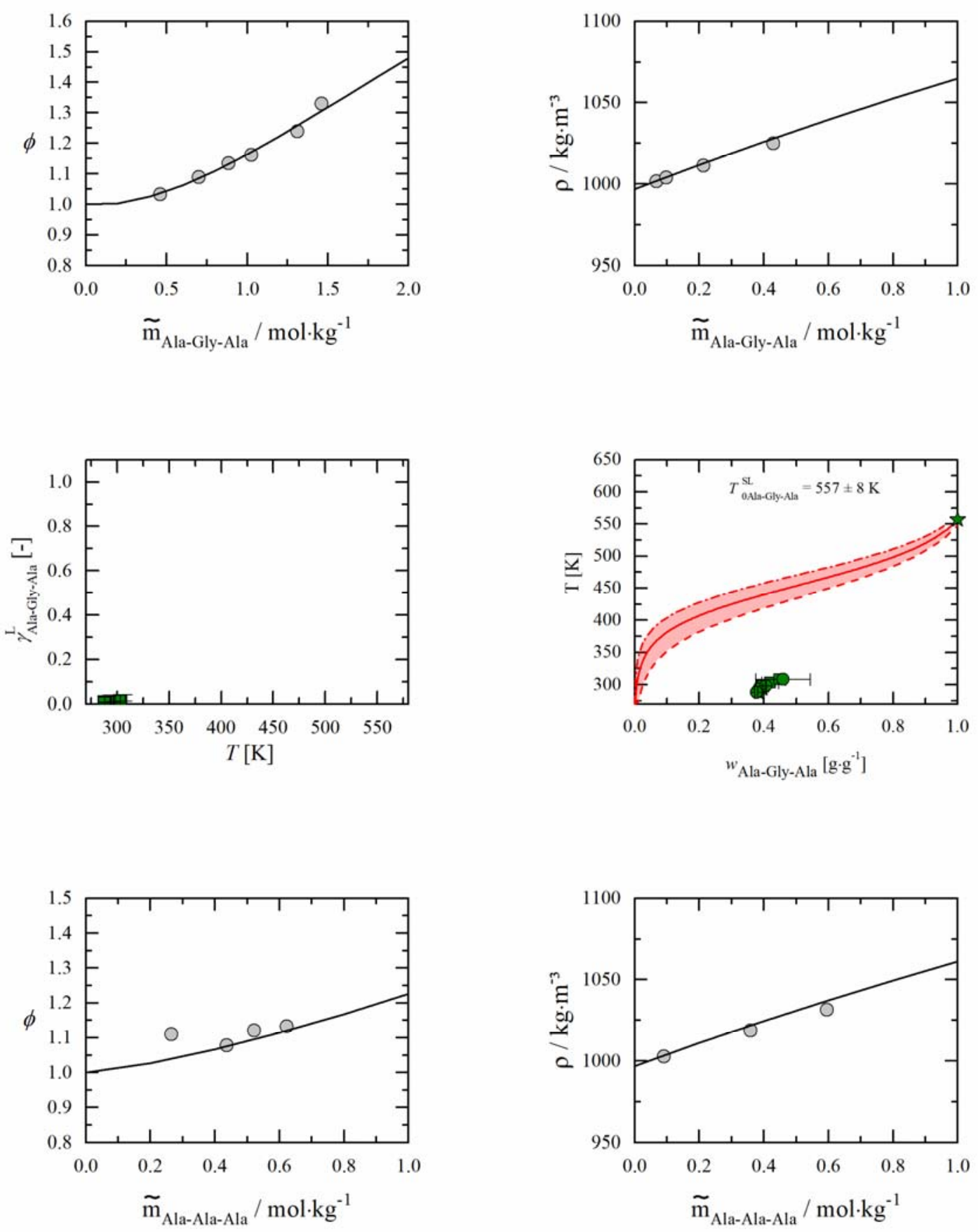

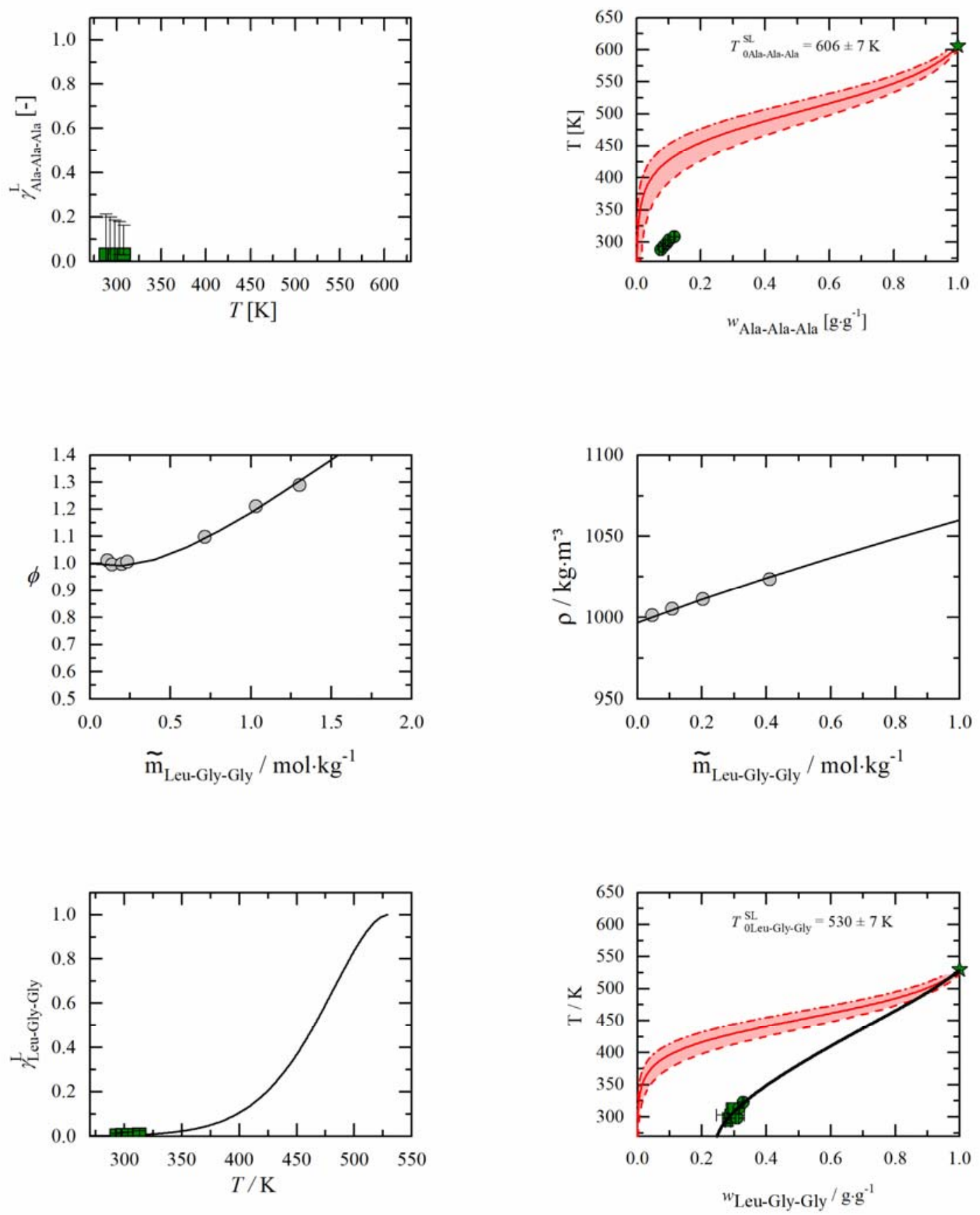

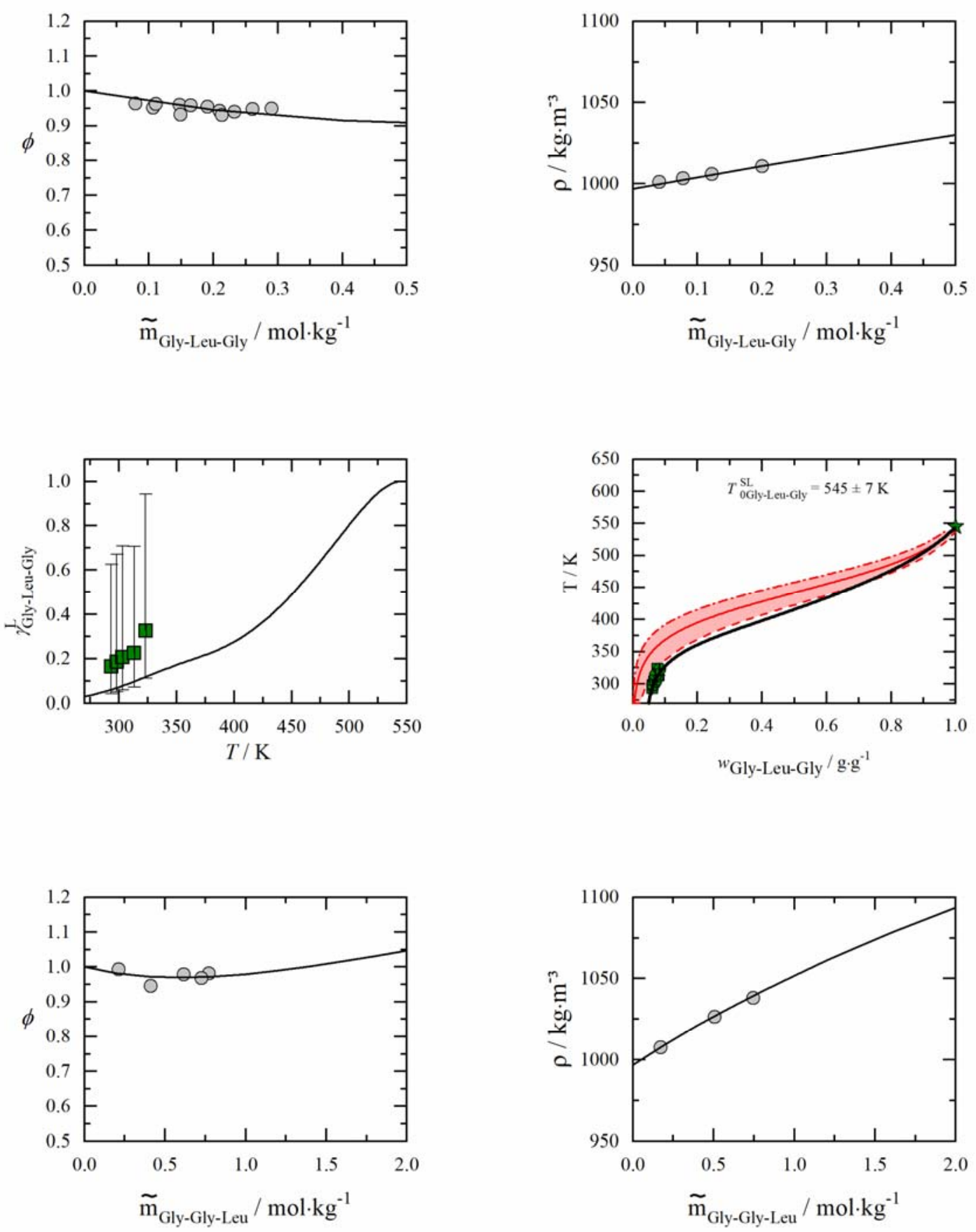

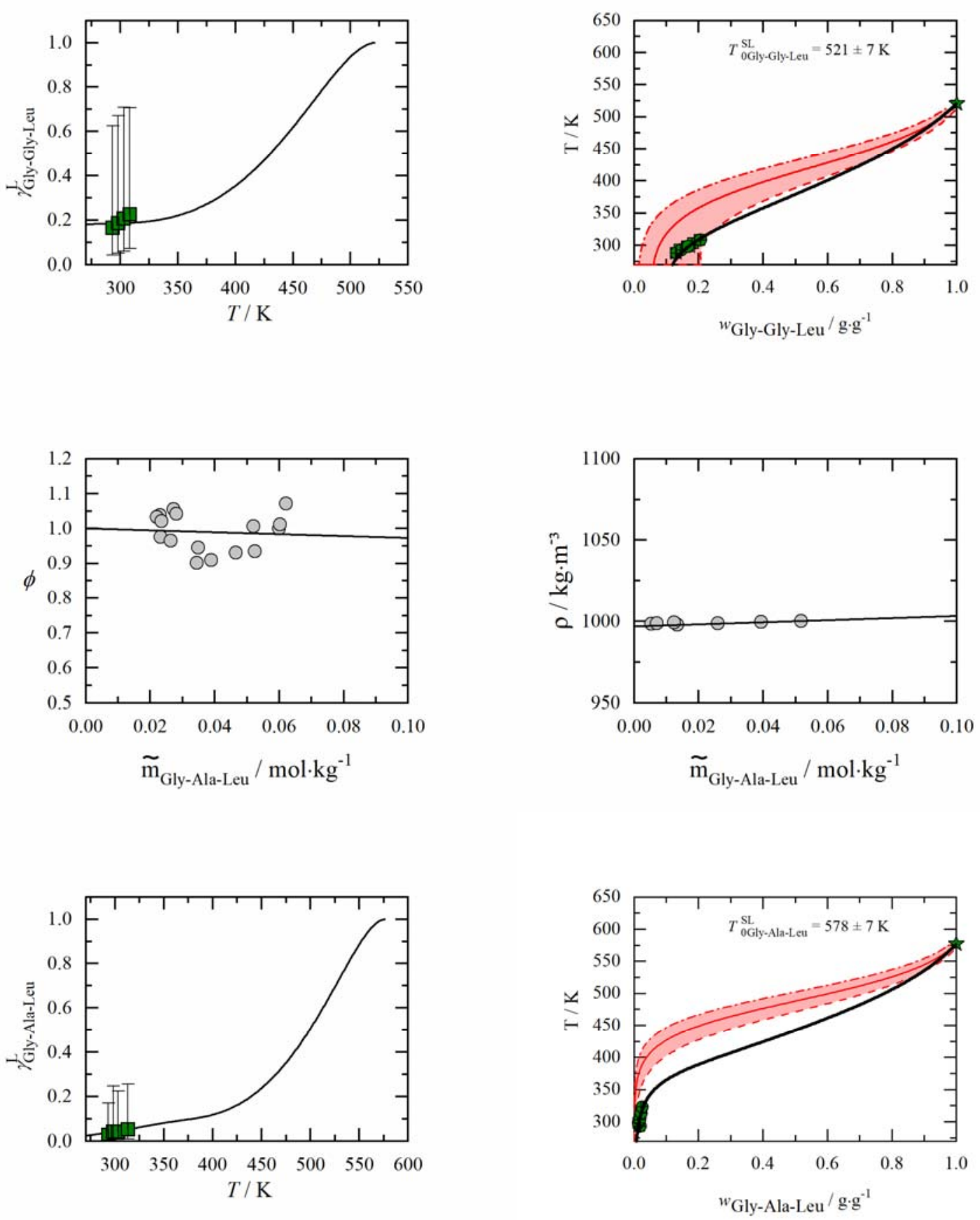

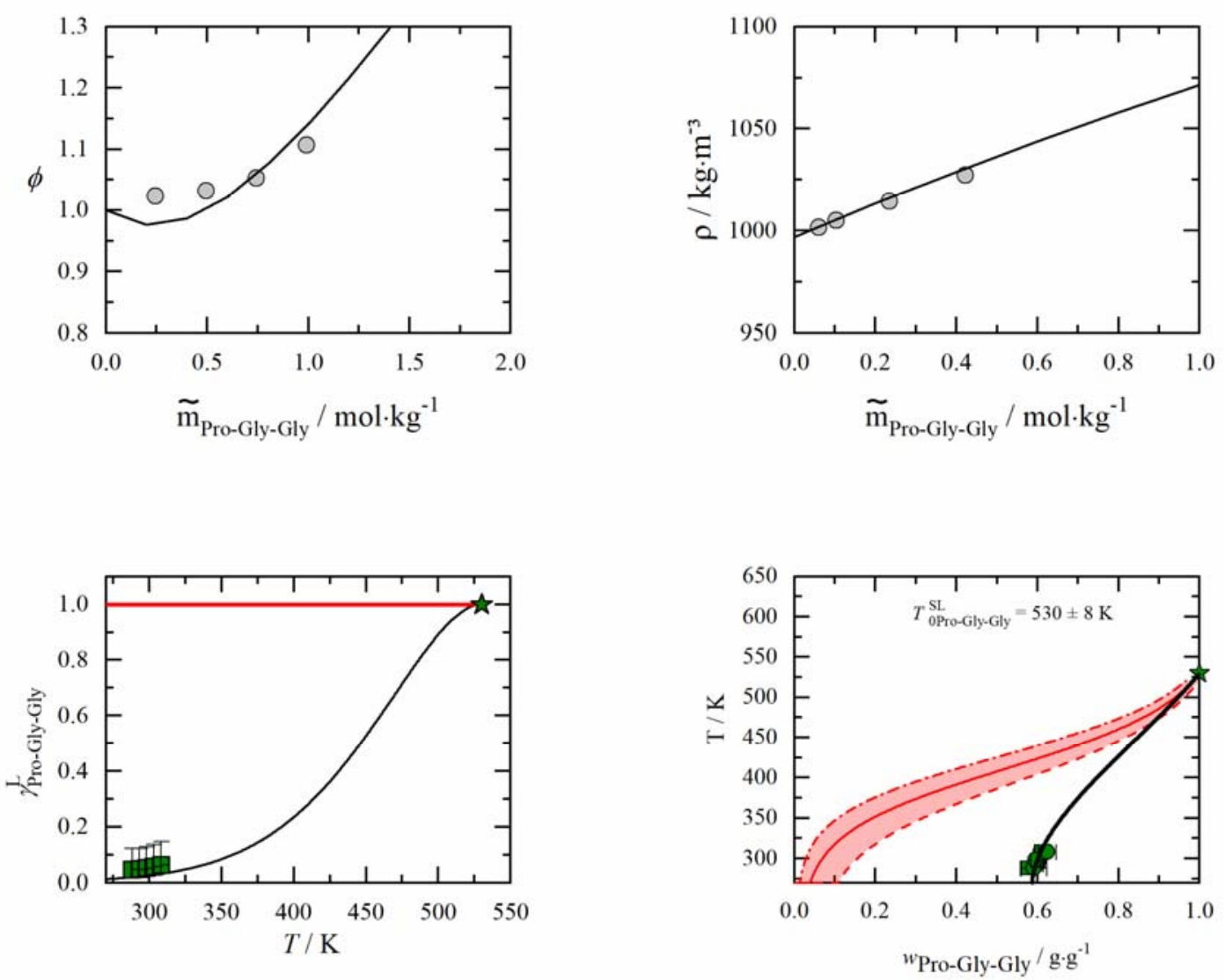

Figure S3 Left top: Osmotic coefficients of peptide + water solutions at T $=273.15 \mathrm{~K}$ and $\mathrm{p}=1 \mathrm{~atm}$. Solid symbols are experimental data from this work. Right top: Densities of peptide + water solutions vs. molality at $\mathrm{T}=273.15 \mathrm{~K}$ and $\mathrm{p}=1 \mathrm{~atm}$. Solid symbols represent experimental data from this work. Left bottom: Activity coefficients vs. temperature diagram. Uncertainties are based on the uncertainties of the melting properties. Red line indicate the activity coefficients $\gamma_{i}^{L}=1$ Right bottom: Aqueous solubility as temperature vs. weight fraction diagram. The red area presents the solubility modeling assuming $\gamma_{i}^{L}=1$ (eq. (1)) in the range of the uncertainties of the melting properties. Symbols present the experimental data from this work: gravimetric method (squares) and photometric method (circles) and the melting temperature (star). The lines are the respective PC-SAFT modeling using the parameters from Table 4 and the melting properties from Table 3. 
Experimental determined osmotic coefficients of peptides in water

Table S5 Osmotic coefficients of dipeptides in aqueous unbuffered solutions at $\mathrm{T}=273.15 \mathrm{~K}$ and $\mathrm{p}=1 \mathrm{~atm}$. Except for Gly-Gly, Gly-Ala, Ala-Gly and Ala-Ala $(\mathrm{T}=298.15 \mathrm{~K}$ and $\mathrm{p}=1 \mathrm{~atm})$

\begin{tabular}{|c|c|c|c|c|c|c|c|}
\hline \multicolumn{2}{|c|}{ Gly-Gly } & \multicolumn{2}{|c|}{ Gly-Ala } & \multicolumn{2}{|c|}{ Ala-Gly } & \multicolumn{2}{|c|}{ Ala-Ala } \\
\hline $\begin{array}{c}\tilde{m} \\
/ \mathrm{mol} \mathrm{kg}^{-1}\end{array}$ & $\phi$ & $\begin{array}{c}\tilde{m} \\
/ \mathrm{mol} \mathrm{kg}^{-1}\end{array}$ & $\phi$ & $\begin{array}{c}\widetilde{m} \\
/ \mathrm{mol} \mathrm{kg}^{-1}\end{array}$ & $\phi$ & $\begin{array}{c}\tilde{m} \\
/ \mathrm{mol} \mathrm{kg}^{-1}\end{array}$ & $\phi$ \\
\hline 0.2 & 0.957 & 0.1 & 0.982 & 0.1 & 0.980 & 0.1 & 0.992 \\
\hline 0.3 & 0.940 & 0.2 & 0.968 & 0.2 & 0.965 & 0.2 & 0.988 \\
\hline 0.5 & 0.914 & 0.3 & 0.957 & 0.3 & 0.954 & 0.3 & 0.988 \\
\hline 0.7 & 0.896 & 0.4 & 0.950 & 0.4 & 0.947 & 0.4 & 0.991 \\
\hline 1 & 0.880 & 0.5 & 0.945 & 0.5 & 0.944 & 0.5 & 0.996 \\
\hline 1.2 & 0.875 & 0.6 & 0.942 & 0.6 & 0.943 & 0.6 & 1.002 \\
\hline 1.5 & 0.868 & 0.7 & 0.941 & 0.7 & 0.943 & 0.7 & 1.010 \\
\hline 1.7 & 0.863 & 0.8 & 0.941 & 0.8 & 0.945 & 0.8 & 1.017 \\
\hline- & - & 0.9 & 0.941 & 0.9 & 0.947 & 0.9 & 1.023 \\
\hline- & - & 1 & 0.942 & 1 & 0.950 & 1.0 & 1.027 \\
\hline \multicolumn{2}{|c|}{ Gly-Ser } & \multicolumn{2}{|c|}{ Ser-Gly } & \multicolumn{2}{|c|}{ Ala-Ser } & \multicolumn{2}{|c|}{ Ser-Ala } \\
\hline $\begin{array}{c}\widetilde{m} \\
/ \mathrm{mol} \mathrm{kg}^{-1}\end{array}$ & $\phi$ & $\begin{array}{c}\tilde{m} \\
/ \mathrm{mol} \mathrm{kg}^{-1}\end{array}$ & $\phi$ & $\begin{array}{c}\widetilde{m} \\
/ \mathrm{mol} \mathrm{kg}^{-1}\end{array}$ & $\phi$ & $\begin{array}{c}\widetilde{m} \\
/ \mathrm{mol} \mathrm{kg}^{-1}\end{array}$ & $\phi$ \\
\hline 0.000 & 1.000 & 0.000 & 1.000 & 0.000 & 1.000 & 0.000 & 1.000 \\
\hline 0.093 & 0.972 & 0.051 & 1.003 & 0.031 & 0.985 & 0.012 & 0.980 \\
\hline 0.123 & 0.964 & 0.078 & 0.995 & 0.085 & 0.985 & 0.020 & 1.040 \\
\hline 0.154 & 0.942 & 0.095 & 0.943 & 0.146 & 0.979 & - & - \\
\hline 0.198 & 0.928 & 0.166 & 0.938 & 0.162 & 0.980 & - & - \\
\hline 0.522 & 0.872 & 0.188 & 0.957 & 0.309 & 0.974 & - & - \\
\hline 0.776 & 0.836 & 0.234 & 0.932 & 0.449 & 0.972 & - & - \\
\hline 1.035 & 0.817 & 0.250 & 0.938 & 0.600 & 0.959 & - & - \\
\hline 1.548 & 0.814 & 0.276 & 0.937 & 0.755 & 0.967 & - & - \\
\hline 2.136 & 0.867 & - & - & - & - & - & - \\
\hline
\end{tabular}

\begin{tabular}{cccccccc}
\hline \multicolumn{2}{c}{ Gly-Pro } & \multicolumn{2}{c}{ Pro-Gly } & \multicolumn{2}{c}{ Ala-Pro } & \multicolumn{2}{c}{ Pro-Ala } \\
\hline $\begin{array}{c}\tilde{m} \\
/ \mathrm{mol} \mathrm{kg}^{-1}\end{array}$ & $\phi$ & $\begin{array}{c}\tilde{m} \\
/ \mathrm{mol} \mathrm{kg}^{-1}\end{array}$ & $\phi$ & $\begin{array}{c}\tilde{m} \\
/ \mathrm{mol} \mathrm{kg}^{-1}\end{array}$ & $\phi$ & $\begin{array}{c}\tilde{m} \\
/ \mathrm{mol} \mathrm{kg}^{-1}\end{array}$ & $\phi$ \\
\hline 0 & 1 & 0 & 1 & 0 & 1 & 0 & 1 \\
0.113 & 0.984 & 0.036 & 0.992 & 0.030 & 1.014 & 0.065 & 0.959 \\
0.208 & 0.948 & 0.317 & 0.940 & 0.052 & 1.025 & 0.131 & 0.945 \\
0.322 & 0.969 & 0.456 & 0.934 & 0.086 & 1.008 & 0.187 & 0.933 \\
0.454 & 0.955 & 0.535 & 0.930 & 0.109 & 1.014 & 0.200 & 0.963 \\
0.641 & 0.978 & 0.614 & 0.924 & 0.144 & 1.037 & 0.208 & 0.949 \\
0.783 & 0.984 & 0.728 & 0.931 & 0.316 & 1.069 & 0.235 & 0.959 \\
1.135 & 1.038 & 0.781 & 0.944 & 0.495 & 1.112 & - & - \\
1.895 & 1.232 & - & - & 0.641 & 1.129 & - & - \\
- & - & - & - & 0.767 & 1.158 & - & - \\
\hline
\end{tabular}

\begin{tabular}{cccccccccc}
\hline \multicolumn{2}{c}{ Gly-Gly-Gly } & \multicolumn{2}{c}{ Gly-Gly-Ala } & \multicolumn{2}{c}{ Gly-Ala-Gly } & \multicolumn{2}{c}{ Ala-Gly-Ala } & & Ala-Ala-Ala \\
\hline $\begin{array}{c}\tilde{m} \\
/ \mathrm{mol} \mathrm{kg}^{-1}\end{array}$ & $\phi$ & $\begin{array}{c}\tilde{m} \\
/ \mathrm{mol} \mathrm{kg}^{-1}\end{array}$ & $\phi$ & $\begin{array}{c}\tilde{m} \\
/ \mathrm{mol} \mathrm{kg}^{-1}\end{array}$ & $\phi$ & $\begin{array}{c}\tilde{m} \\
/ \mathrm{mol} \mathrm{kg}^{-1}\end{array}$ & $\phi$ & $\begin{array}{c}\tilde{m} \\
/ \mathrm{mol} \mathrm{kg}^{-1}\end{array}$ & $\phi$ \\
\hline 0.000 & 1.000 & 0.000 & 1.000 & 0.000 & 1.000 & 0.000 & 1.000 & 0.000 & 1.000 \\
0.077 & 0.963 & 0.075 & 0.988 & 0.092 & 1.012 & 0.461 & 1.033 & 0.265 & 1.109
\end{tabular}




\begin{tabular}{ccccccccccc}
0.084 & 0.934 & 0.095 & 0.991 & 0.113 & 1.032 & 0.700 & 1.089 & 0.437 & 1.078 \\
0.162 & 0.905 & 0.130 & 0.971 & 0.115 & 1.038 & 0.885 & 1.135 & 0.521 & 1.120 \\
0.175 & 0.905 & 0.142 & 0.976 & 0.129 & 1.032 & 1.027 & 1.162 & 0.623 & 1.132 \\
0.227 & 0.877 & 0.174 & 0.970 & 0.172 & 1.032 & 1.313 & 1.239 & - & - \\
0.291 & 0.860 & 0.178 & 0.948 & 0.190 & 1.031 & 1.460 & 1.330 & - & - \\
- & - & 0.214 & 0.956 & 0.216 & 1.029 & - & - & - & - \\
- & - & 0.236 & 0.941 & 0.238 & 1.009 & - & - & - & - \\
- & - & 0.284 & 0.939 & 0.239 & 1.013 & - & - & - & - \\
\hline
\end{tabular}

\begin{tabular}{|c|c|c|c|c|c|c|c|c|c|}
\hline \multicolumn{2}{|c|}{ Leu-Gly-Gly } & \multicolumn{2}{|c|}{ Gly-Leu-Gly } & \multicolumn{2}{|c|}{ Gly-Gly-Leu } & \multicolumn{2}{|c|}{ Pro-Gly-Gly } & \multicolumn{2}{|c|}{ Gly-Ala-Leu } \\
\hline $\begin{array}{c}\widetilde{m} \\
/ \mathrm{mol} \mathrm{kg}^{-1} \\
\end{array}$ & $\phi$ & $\begin{array}{c}\widetilde{m} \\
/ \mathrm{mol} \mathrm{kg}^{-1} \\
\end{array}$ & $\phi$ & $\begin{array}{c}\widetilde{m} \\
/ \mathrm{mol} \mathrm{kg}^{-1}\end{array}$ & $\phi$ & $\begin{array}{c}\widetilde{m} \\
/ \mathrm{mol} \mathrm{kg}^{-1}\end{array}$ & $\phi$ & $\begin{array}{c}\widetilde{m} \\
/ \mathrm{mol} \mathrm{kg}^{-1}\end{array}$ & $\phi$ \\
\hline 0.000 & 1.000 & 0.000 & 1.000 & 0.000 & 1.000 & 0.000 & 1.000 & 0.000 & 1.000 \\
\hline 0.110 & 1.012 & 0.079 & 0.964 & 0.213 & 0.993 & 0.246 & 1.023 & 0.024 & 1.022 \\
\hline 0.137 & 0.996 & 0.107 & 0.952 & 0.412 & 0.945 & 0.494 & 1.032 & 0.035 & 0.945 \\
\hline 0.197 & 0.998 & 0.111 & 0.962 & 0.617 & 0.979 & 0.743 & 1.053 & 0.052 & 1.006 \\
\hline 0.233 & 1.006 & 0.147 & 0.960 & 0.773 & 0.981 & 0.991 & 1.106 & 0.060 & 1.012 \\
\hline 0.715 & 1.099 & 0.149 & 0.932 & 0.726 & 0.968 & - & - & - & - \\
\hline 1.032 & 1.210 & 0.165 & 0.959 & - & - & - & - & - & - \\
\hline 1.303 & 1.289 & 0.191 & 0.955 & - & - & - & - & - & - \\
\hline- & - & 0.210 & 0.942 & - & - & - & - & - & - \\
\hline - & - & 0.213 & 0.931 & - & - & - & - & - & - \\
\hline- & - & 0.233 & 0.941 & - & - & - & - & - & - \\
\hline- & - & 0.261 & 0.948 & - & - & - & - & - & - \\
\hline- & - & 0.290 & 0.949 & - & - & - & - & - & - \\
\hline
\end{tabular}


Experimental determined mixture density of peptides in water

Table S6 Density of peptides in aqueous unbuffered solutions at $\mathrm{T}=298.15 \mathrm{~K}$ and $\mathrm{p}=1 \mathrm{~atm}$.

\begin{tabular}{|c|c|c|c|c|c|c|c|}
\hline \multicolumn{2}{|c|}{ Gly-Gly } & \multicolumn{2}{|c|}{ Gly-Ala } & \multicolumn{2}{|c|}{ Ala-Gly } & \multicolumn{2}{|c|}{ Ala-Ala } \\
\hline $\begin{array}{c}\tilde{m} \\
/ \mathrm{mol} \mathrm{kg}^{-1}\end{array}$ & $\begin{array}{c}\rho \\
/ \mathrm{kg} \mathrm{m}^{-3}\end{array}$ & $\begin{array}{c}\tilde{m} \\
/ \mathrm{mol} \mathrm{kg}^{-1}\end{array}$ & $\begin{array}{c}\rho \\
/ \mathrm{kg} \mathrm{m}^{-3}\end{array}$ & $\begin{array}{c}\tilde{m} \\
/ \mathrm{mol} \mathrm{kg}^{-1}\end{array}$ & $\begin{array}{c}\rho \\
/ \mathrm{kg} \mathrm{m}^{-3}\end{array}$ & $\begin{array}{c}\tilde{m} \\
/ \mathrm{mol} \mathrm{kg}^{-1}\end{array}$ & $\begin{array}{c}\rho \\
/ \mathrm{kg} \mathrm{m}^{-3}\end{array}$ \\
\hline 0.049 & 999.8 & 0.238 & 1009.4 & 0.155 & 1004.9 & 0.167 & 1005.0 \\
\hline 0.100 & 1002.6 & 0.362 & 1015.6 & 0.137 & 1004.0 & 0.234 & 1008.2 \\
\hline 0.152 & 1005.4 & 0.453 & 1020.0 & 0.123 & 1003.3 & 0.324 & 1012.3 \\
\hline 0.198 & 1007.9 & 0.677 & 1030.5 & 0.106 & 1002.5 & 0.465 & 1018.6 \\
\hline 0.248 & 1010.5 & 0.776 & 1034.9 & 0.087 & 1001.5 & 0.657 & 1026.9 \\
\hline 0.300 & 1013.3 & 0.975 & 1043.6 & 0.071 & 1000.7 & 0.805 & 1032.8 \\
\hline 0.346 & 1015.6 & 1.033 & 1045.9 & 0.055 & 999.9 & 0.943 & 1038.2 \\
\hline 0.395 & 1018.2 & 1.366 & 1059.2 & 0.039 & 999.1 & - & - \\
\hline 0.445 & 1020.7 & 1.559 & 1066.4 & 0.030 & 998.6 & - & - \\
\hline 0.490 & 1023.4 & - & - & - & - & - & - \\
\hline 0.543 & 1025.6 & - & - & - & - & - & - \\
\hline- & - & - & - & - & - & - & - \\
\hline \multicolumn{2}{|c|}{ Gly-Ser } & \multicolumn{2}{|c|}{ Ser-Gly } & \multicolumn{2}{|c|}{ Ala-Ser } & \multicolumn{2}{|c|}{ Ser-Ala } \\
\hline $\begin{array}{c}\tilde{m} \\
/ \mathrm{mol} \mathrm{kg}^{-1}\end{array}$ & $\begin{array}{c}\rho \\
/ \mathrm{kg} \mathrm{m}^{-3}\end{array}$ & $\begin{array}{c}\tilde{m} \\
/ \mathrm{mol} \mathrm{kg}^{-1}\end{array}$ & $\begin{array}{c}\rho \\
/ \mathrm{kg} \mathrm{m}^{-3}\end{array}$ & $\begin{array}{c}\tilde{m} \\
/ \mathrm{mol} \mathrm{kg}^{-1}\end{array}$ & $\begin{array}{c}\rho \\
/ \mathrm{kg} \mathrm{m}^{-3}\end{array}$ & $\begin{array}{c}\tilde{m} \\
/ \mathrm{mol} \mathrm{kg}^{-1}\end{array}$ & $\begin{array}{c}\rho \\
/ \mathrm{kg} \mathrm{m}^{-3}\end{array}$ \\
\hline 0.031 & 999.0 & 0.051 & 1000.4 & 0.028 & 999.0 & 0.002 & 997.2 \\
\hline 0.061 & 1001.0 & 0.102 & 1003.7 & 0.055 & 1001.0 & 0.007 & 997.5 \\
\hline 0.092 & 1003.0 & 0.157 & 1007.2 & 0.083 & 1003.0 & 0.011 & 997.8 \\
\hline 0.137 & 1006.0 & 0.210 & 1010.6 & 0.125 & 1005.0 & 0.016 & 998.1 \\
\hline- & - & - & - & - & - & 0.020 & 998.2 \\
\hline \multicolumn{2}{|c|}{ Gly-Pro } & \multicolumn{2}{|c|}{ Pro-Gly } & \multicolumn{2}{|c|}{ Ala-Pro } & \multicolumn{2}{|c|}{ Pro-Ala } \\
\hline $\begin{array}{c}\tilde{m} \\
/ \mathrm{mol} \mathrm{kg}^{-1}\end{array}$ & $\begin{array}{c}\rho \\
/ \mathrm{kg} \mathrm{m}^{-3}\end{array}$ & $\begin{array}{c}\tilde{m} \\
/ \mathrm{mol} \mathrm{kg}^{-1}\end{array}$ & $\begin{array}{c}\rho \\
/ \mathrm{kg} \mathrm{m}^{-3}\end{array}$ & $\begin{array}{c}\tilde{m} \\
/ \mathrm{mol} \mathrm{kg}^{-1}\end{array}$ & $\begin{array}{c}\rho \\
/ \mathrm{kg} \mathrm{m}^{-3}\end{array}$ & $\begin{array}{c}\tilde{m} \\
/ \mathrm{mol} \mathrm{kg}^{-1}\end{array}$ & $\begin{array}{c}\rho \\
/ \mathrm{kg} \mathrm{m}^{-3}\end{array}$ \\
\hline 0.179 & 1007.1 & 0.043 & 999.0 & 0.039 & 999.0 & 0.044 & 999.3 \\
\hline 0.562 & 1027.1 & 0.085 & 1001.0 & 0.078 & 1001.0 & 0.088 & 1001.5 \\
\hline 1.173 & 1054.9 & 0.116 & 1003.0 & 0.095 & 1002.0 & 0.133 & 1003.5 \\
\hline 1.708 & 1076.1 & 0.144 & 1005.0 & 0.147 & 1005.0 & 0.186 & 1006.4 \\
\hline
\end{tabular}

\begin{tabular}{|c|c|c|c|c|c|c|c|c|c|}
\hline \multicolumn{2}{|c|}{ Gly-Gly-Gly } & \multicolumn{2}{|c|}{ Gly-Gly-Ala } & \multicolumn{2}{|c|}{ Gly-Ala-Gly } & \multicolumn{2}{|c|}{ Ala-Gly-Ala } & \multicolumn{2}{|c|}{ Ala-Ala-Ala } \\
\hline $\begin{array}{c}\tilde{m} \\
/ \mathrm{mol} \mathrm{kg}^{-1}\end{array}$ & $\begin{array}{c}\rho \\
/ \mathrm{kg} \mathrm{m}^{-3}\end{array}$ & $\begin{array}{c}\tilde{m} \\
/ \mathrm{mol} \mathrm{kg}^{-1}\end{array}$ & $\begin{array}{c}\rho \\
/ \mathrm{kg} \mathrm{m}^{-3}\end{array}$ & $\begin{array}{c}\tilde{m} \\
/ \mathrm{mol} \mathrm{kg}^{-1}\end{array}$ & $\begin{array}{c}\rho \\
/ \mathrm{kg} \mathrm{m}^{-3}\end{array}$ & $\begin{array}{c}\tilde{m} \\
/ \mathrm{mol} \mathrm{kg}^{-1}\end{array}$ & $\begin{array}{c}\rho \\
/ \mathrm{kg} \mathrm{m}^{-3}\end{array}$ & $\begin{array}{c}\tilde{m} \\
/ \mathrm{mol} \mathrm{kg}^{-1}\end{array}$ & $\begin{array}{c}\rho \\
/ \mathrm{kg} \mathrm{m}^{-3}\end{array}$ \\
\hline 0.079 & 1004.7 & 0.049 & 1002.2 & 0.024 & 1000.1 & 0.067 & 1001.7 & 0.091 & 1002.8 \\
\hline 0.158 & 1010.4 & 0.0966 & 1005.3 & 0.075 & 1003.5 & 0.098 & 1003.8 & 0.358 & 1018.7 \\
\hline 0.291 & 1019.9 & 0.2435 & 1012.7 & 0.146 & 1008.4 & 0.213 & 1011.4 & 0.595 & 1031.6 \\
\hline- & - & - & - & 0.247 & 1015.2 & 0.429 & 1024.9 & - & - \\
\hline \multicolumn{2}{|c|}{ Leu-Gly-Gly } & \multicolumn{2}{|c|}{ Gly-Leu-Gly } & \multicolumn{2}{|c|}{ Gly-Gly-Leu } & \multicolumn{2}{|c|}{ Pro-Gly-Gly } & \multicolumn{2}{|c|}{ Gly-Ala-Leu } \\
\hline $\begin{array}{c}\tilde{m} \\
/ \mathrm{mol} \mathrm{kg}^{-1} \\
\end{array}$ & $\begin{array}{c}\rho \\
/ \mathrm{kg} \mathrm{m}^{-3} \\
\end{array}$ & $\begin{array}{c}\widetilde{m} \\
/ \mathrm{mol} \mathrm{kg}^{-1} \\
\end{array}$ & $\begin{array}{c}\rho \\
/ \mathrm{kg} \mathrm{m}^{-3} \\
\end{array}$ & $\begin{array}{c}\tilde{m} \\
/ \mathrm{mol} \mathrm{kg}^{-1}\end{array}$ & $\begin{array}{c}\rho \\
/ \mathrm{kg} \mathrm{m}^{-3} \\
\end{array}$ & $\begin{array}{c}\tilde{m} \\
/ \mathrm{mol} \mathrm{kg}^{-1} \\
\end{array}$ & $\begin{array}{c}\rho \\
/ \mathrm{kg} \mathrm{m}^{-3} \\
\end{array}$ & $\begin{array}{c}\widetilde{m} \\
/ \mathrm{mol} \mathrm{kg}^{-1} \\
\end{array}$ & $\begin{array}{c}\rho \\
/ \mathrm{kg} \mathrm{m}^{-3} \\
\end{array}$ \\
\hline 0.046 & 1001.4 & 0.041 & 1001.1 & 0.172 & 1007.7 & 0.060 & 1001.5 & 0.013 & 997.9 \\
\hline 0.108 & 1005.3 & 0.078 & 1003.3 & 0.508 & 1026.3 & 0.104 & 1004.9 & 0.026 & 998.7 \\
\hline 0.204 & 1011.3 & 0.122 & 1005.9 & 0.747 & 1038.1 & 0.234 & 1014.3 & 0.039 & 999.7 \\
\hline 0.411 & 1023.6 & 0.201 & 1010.8 & - & - & 0.422 & 1027.1 & 0.052 & 1000.3 \\
\hline
\end{tabular}




\section{Experimental determined peptide solubility}

Table S7 The weight fraction solubility of peptides in aqueous unbuffered solutions at different temperatures (gravimetric method) and $\mathrm{p}=1 \mathrm{~atm}$. The measured $\mathrm{pH}$ values of the solutions are listed in Table S4

\begin{tabular}{|c|c|c|c|c|c|c|c|}
\hline Peptide & $288.15 \mathrm{~K}$ & $293.15 \mathrm{~K}$ & $298.15 K$ & $303.15 \mathrm{~K}$ & $308.15 \mathrm{~K}$ & $313.15 \mathrm{~K}$ & $323.15 K$ \\
\hline Gly-Gly & & $0.184 \pm 0.003$ & $0.195 \pm 0.004$ & & $0.227 \pm 0.015$ & - & \\
\hline Gly-Ala & & $0.352 \pm 0.007$ & $0.404 \pm 0.041$ & $0.419 \pm 0.215$ & & $0.452 \pm 0.037$ & $0.459 \pm 0.074$ \\
\hline Ala-Gly & & & $0.184 \pm 0.013$ & & & & \\
\hline Ala-Ala & & & & & & & $0.323 \pm 0.01$ \\
\hline Gly-Ser & & & $0.323 \pm 0.017$ & & & & \\
\hline Ser-Gly & $0.035 \pm 0.002$ & $0.042 \pm 0.006$ & $0.046 \pm 0.001$ & $0.061 \pm 0.001$ & $0.058 \pm 0.001$ & & \\
\hline Ala-Ser & & & & $0.406 \pm 0.014$ & & & $0.531 \pm 0.001$ \\
\hline Ser-Ala & $0.003 \pm 0.001$ & $0.004 \pm 0.001$ & $0.004 \pm 0.001$ & $0.005 \pm 0.001$ & $0.004 \pm 0.001$ & & \\
\hline Gly-Pro & & & $0.655 \pm 0.046$ & & & & \\
\hline Pro-Gly & & & $0.203 \pm 0.005$ & & & & $0.259 \pm 0.005$ \\
\hline Ala-Pro & & & $0.627 \pm 0.014$ & & & & $0.629 \pm 0.025$ \\
\hline Pro-Ala & $0.048 \pm 0.015$ & $0.051 \pm 0.004$ & $0.054 \pm 0.001$ & $0.059 \pm 0.001$ & & $0.059 \pm 0.001$ & \\
\hline Gly-Gly-Gly & & & $0.058 \pm 0.002$ & $0.076 \pm 0.001$ & & $0.092 \pm 0.001$ & \\
\hline Gly-Gly-Ala & & & $0.045 \pm 0.003$ & $0.074 \pm 0.004$ & & $0.08 \pm 0.001$ & $0.079 \pm 0.001$ \\
\hline Gly-Ala-Gly & & & $0.049 \pm 0.001$ & & & $0.06 \pm 0.001$ & $0.073 \pm 0.001$ \\
\hline Ala-Gly-Ala & $0.377 \pm 0.026$ & $0.384 \pm 0.018$ & $0.406 \pm 0.001$ & & $0.459 \pm 0.001$ & & \\
\hline Ala-Ala-Ala & & & & & & & \\
\hline Leu-Gly-Gly & & & $0.31 \pm 0.011$ & & & $0.315 \pm 0.001$ & $0.328 \pm 0.001$ \\
\hline Gly-Leu-Gly & & & $0.062 \pm 0.012$ & $0.07 \pm 0.004$ & & $0.071 \pm 0.001$ & $0.083 \pm 0.001$ \\
\hline Gly-Gly-Leu & $0.13 \pm 0.005$ & $0.147 \pm 0.002$ & $0.168 \pm 0.001$ & & $0.206 \pm 0.001$ & & \\
\hline Pro-Gly-Gly & $0.591 \pm 0.015$ & & $0.595 \pm 0.016$ & & $0.625 \pm 0.001$ & & \\
\hline Gly-Ala-Leu & & $0.02 \pm 0.001$ & $0.013 \pm 0.001$ & $0.018 \pm 0.001$ & & $0.023 \pm 0.001$ & $0.025 \pm 0.001$ \\
\hline
\end{tabular}

Table S8 The weight fraction solubility of peptides in aqueous unbuffered solutions at different temperatures (photometric method) and $\mathrm{p}=1 \mathrm{~atm}$. The measured $\mathrm{pH}$ values of the solutions are listed in Table S4

\begin{tabular}{|c|c|c|c|c|c|c|c|}
\hline Peptide & $288.15 \mathrm{~K}$ & $293.15 \mathrm{~K}$ & $298.15 \mathrm{~K}$ & $303.15 \mathrm{~K}$ & $308.15 \mathrm{~K}$ & $313.15 \mathrm{~K}$ & $323.15 K$ \\
\hline \multicolumn{8}{|l|}{ Gly-Gly } \\
\hline \multicolumn{8}{|l|}{ Gly-Ala } \\
\hline Ala-Gly & & $0.169 \pm 0.004$ & $0.175 \pm 0.001$ & $0.188 \pm 0.013$ & & $0.189 \pm 0.005$ & $0.196 \pm 0.004$ \\
\hline Gly-Ser & & $0.277 \pm 0.001$ & $0.289 \pm 0.011$ & $0.296 \pm 0.005$ & & $0.308 \pm 0.017$ & $0.32 \pm 0.011$ \\
\hline Ser-Gly & $0.033 \pm 0.001$ & $0.038 \pm 0.001$ & $0.043 \pm 0.001$ & $0.054 \pm 0.002$ & & & \\
\hline Gly-Pro & & $0.641 \pm 0.128$ & $0.664 \pm 0.124$ & $0.675 \pm 0.046$ & & $0.694 \pm 0.066$ & $0.705 \pm 0.048$ \\
\hline Pro-Gly & & & $0.194 \pm 0.008$ & & & $0.221 \pm 0.005$ & $0.243 \pm 0.005$ \\
\hline Ala-Pro & & $0.498 \pm 0.041$ & $0.514 \pm 0.015$ & $0.54 \pm 0.009$ & & $0.539 \pm 0.014$ & $0.572 \pm 0.025$ \\
\hline Pro-Ala & $0.048 \pm 0.002$ & $0.049 \pm 0.001$ & $0.052 \pm 0.001$ & $0.056 \pm 0.005$ & & & \\
\hline Gly-Gly-Gly & & $0.056 \pm 0.001$ & $0.061 \pm 0.001$ & $0.07 \pm 0.001$ & & $0.086 \pm 0.002$ & $0.102 \pm 0.001$ \\
\hline Leu-Gly-Gly & & $0.282 \pm 0.009$ & $0.281 \pm 0.003$ & $0.289 \pm 0.043$ & & $0.293 \pm 0.011$ & \\
\hline Gly-Leu-Gly & & $0.061 \pm 0.001$ & $0.063 \pm 0.001$ & $0.066 \pm 0.002$ & & $0.082 \pm 0.012$ & $0.077 \pm 0.004$ \\
\hline Gly-Gly-Leu & $0.13 \pm 0.016$ & $0.142 \pm 0.004$ & $0.168 \pm 0.004$ & $0.184 \pm 0.005$ & $0.203 \pm 0.002$ & & \\
\hline Pro-Gly-Gly & $0.577 \pm 0.017$ & $0.598 \pm 0.017$ & $0.603 \pm 0.015$ & $0.607 \pm 0.015$ & $0.61 \pm 0.016$ & & \\
\hline Gly-Ala-Leu & & $0.018 \pm 0.001$ & $0.014 \pm 0.001$ & $0.018 \pm 0.001$ & & $0.022 \pm 0.0010$ & \\
\hline
\end{tabular}


Table S9 The weight fraction solubility of peptides in aqueous solutions at different temperatures (gravimetric method) and $\mathrm{p}=1 \mathrm{~atm}$. The solubilities are shifted to $\mathrm{pH}=7$ using the Henderson Hasselbalch equation $\mathrm{S} 1$ based on the $\mathrm{pK}$ values from Table S3, the $\mathrm{pH}$ values measured in this work. The measured $\mathrm{pH}$ values of the solutions are listed in Table S4.

\begin{tabular}{|c|c|c|c|c|c|c|c|}
\hline Peptide & $288.15 \mathrm{~K}$ & $293.15 \mathrm{~K}$ & $298.15 \mathrm{~K}$ & $303.15 \mathrm{~K}$ & $308.15 \mathrm{~K}$ & $313.15 \mathrm{~K}$ & $323.15 K$ \\
\hline Gly-Gly & & $0.194 \pm 0.003$ & $0.209 \pm 0.004$ & & $0.248 \pm 0.015$ & - & \\
\hline Gly-Ala & & $0.184 \pm 0.003$ & $0.434 \pm 0.043$ & $0.435 \pm 0.022$ & & $0.468 \pm 0.039$ & $0.475 \pm 0.078$ \\
\hline Ala-Gly & & & $0.189 \pm 0.002$ & & & & \\
\hline Ala-Ala & & & & & & & $0.323 \pm 0.01$ \\
\hline Gly-Ser & & & $0.337 \pm 0.01$ & & & & \\
\hline Ser-Gly & $0.04 \pm 0.001$ & $0.047 \pm 0.001$ & $0.052 \pm 0.001$ & $0.067 \pm 0.002$ & $0.062 \pm 0.005$ & & \\
\hline Ala-Ser & & $0.328 \pm 0.034$ & $0.34 \pm 0.035$ & $0.364 \pm 0.036$ & $0.371 \pm 0.037$ & $0.334 \pm 0.035$ & \\
\hline Ser-Ala & $0.004 \pm 0.001$ & $0.004 \pm 0.001$ & $0.005 \pm 0.001$ & $0.005 \pm 0.001$ & $0.005 \pm 0.001$ & & \\
\hline Gly-Pro & & & $0.66 \pm 0.05$ & & & & \\
\hline Pro-Gly & & & $0.203 \pm 0.013$ & & & & $0.259 \pm 0.005$ \\
\hline Ala-Pro & & & $0.635 \pm 0.06$ & & & & $0.636 \pm 0.036$ \\
\hline Pro-Ala & & $0.049 \pm 0.002$ & $0.052 \pm 0.002$ & $0.056 \pm 0.002$ & & & \\
\hline Gly-Gly-Gly & & & $0.065 \pm 0.004$ & $0.085 \pm 0.008$ & & $0.103 \pm 0.006$ & \\
\hline Gly-Gly-Ala & & & $0.05 \pm 0.002$ & $0.083 \pm 0.012$ & & $0.088 \pm 0.01$ & $0.088 \pm 0.002$ \\
\hline Gly-Ala-Gly & & & $0.052 \pm 0.001$ & & & $0.076 \pm 0.003$ & $0.076 \pm 0.003$ \\
\hline Ala-Gly-Ala & $0.394 \pm 0.009$ & $0.401 \pm 0.017$ & $0.423 \pm 0.007$ & & $0.477 \pm 0.006$ & & \\
\hline Ala-Ala-Ala & & $0.157 \pm 0.009$ & $0.067 \pm 0.008$ & & & & \\
\hline Leu-Gly-Gly & & & $0.323 \pm 0.005$ & & & $0.328 \pm 0.003$ & $0.341 \pm 0.003$ \\
\hline Gly-Leu-Gly & & & $0.069 \pm 0.002$ & $0.077 \pm 0.005$ & & $0.078 \pm 0.001$ & $0.091 \pm 0.001$ \\
\hline Gly-Gly-Leu & $0.145 \pm 0.004$ & $0.162 \pm 0.007$ & $0.185 \pm 0.003$ & & $0.226 \pm 0.003$ & & \\
\hline Pro-Gly-Gly & $0.592 \pm 0.043$ & & $0.596 \pm 0.02$ & & $0.625 \pm 0.033$ & & \\
\hline Gly-Ala-Leu & & $0.022 \pm 0.001$ & $0.015 \pm 0.001$ & $0.021 \pm 0.001$ & & $0.025 \pm 0.001$ & $0.028 \pm 0.003$ \\
\hline
\end{tabular}

Table S10 The weight fraction solubility of peptides in aqueous solutions at different temperatures (photometric method) and $\mathrm{p}=1 \mathrm{~atm}$. The solubilities are shifted to $\mathrm{pH}=7$ using the Henderson Hasselbalch equation $\mathrm{S} 1$ based on the $\mathrm{pK}_{\mathrm{a}}$ values from Table $\mathrm{S} 3$, the $\mathrm{pH}$ values measured in this work. The measured $\mathrm{pH}$ values of the solutions are listed in Table S4.

\begin{tabular}{|c|c|c|c|c|c|c|c|}
\hline Peptide & $288.15 \mathrm{~K}$ & $293.15 \mathrm{~K}$ & $298.15 \mathrm{~K}$ & $303.15 \mathrm{~K}$ & $308.15 \mathrm{~K}$ & $313.15 \mathrm{~K}$ & $323.15 \mathrm{~K}$ \\
\hline \multicolumn{8}{|l|}{ Gly-Gly } \\
\hline \multicolumn{8}{|l|}{ Gly-Ala } \\
\hline Ala-Gly & & $0.174 \pm 0.015$ & $0.18 \pm 0.015$ & $0.194 \pm 0.016$ & & $0.195 \pm 0.016$ & $0.201 \pm 0.016$ \\
\hline Ala-Ala & & $0.295 \pm 0.02$ & $0.29 \pm 0.02$ & $0.296 \pm 0.02$ & & $0.303 \pm 0.02$ & $0.291 \pm 0.02$ \\
\hline Gly-Ser & & $0.284 \pm 0.018$ & $0.296 \pm 0.019$ & $0.303 \pm 0.019$ & & $0.316 \pm 0.019$ & $0.328 \pm 0.02$ \\
\hline Ser-Gly & $0.037 \pm 0.002$ & $0.043 \pm 0.002$ & $0.048 \pm 0.003$ & $0.059 \pm 0.003$ & $0.059 \pm 0.003$ & & \\
\hline Ala-Ser & & & & $0.416 \pm 0.012$ & & & $0.539 \pm 0.002$ \\
\hline Ser-Ala & $0.004 \pm 0.001$ & $0.004 \pm 0.001$ & $0.005 \pm 0.001$ & $0.005 \pm 0.001$ & $0.005 \pm 0.001$ & & \\
\hline Gly-Pro & & $0.646 \pm 0.287$ & $0.662 \pm 0.287$ & $0.678 \pm 0.288$ & & $0.69 \pm 0.288$ & $0.703 \pm 0.288$ \\
\hline Pro-Gly & & & $0.191 \pm 0.01$ & & & $0.217 \pm 0.011$ & $0.239 \pm 0.011$ \\
\hline Ala-Pro & & $0.505 \pm 0.224$ & $0.522 \pm 0.222$ & $0.547 \pm 0.119$ & & $0.547 \pm 0.223$ & $0.579 \pm 0.225$ \\
\hline Pro-Ala & & $0.051 \pm 0.001$ & $0.053 \pm 0.001$ & $0.059 \pm 0.001$ & & & \\
\hline Gly-Gly-Gly & & $0.064 \pm 0.003$ & $0.07 \pm 0.003$ & $0.079 \pm 0.003$ & & $0.098 \pm 0.003$ & $0.116 \pm 0.003$ \\
\hline Gly-Gly-Ala & & $0.049 \pm 0.002$ & $0.053 \pm 0.002$ & $0.057 \pm 0.003$ & & $0.077 \pm 0.003$ & $0.089 \pm 0.003$ \\
\hline Gly-Ala-Gly & & $0.047 \pm 0.003$ & $0.051 \pm 0.003$ & $0.053 \pm 0.003$ & & $0.058 \pm 0.003$ & $0.07 \pm 0.004$ \\
\hline Ala-Gly-Ala & $0.403 \pm 0.054$ & $0.409 \pm 0.054$ & $0.414 \pm 0.054$ & $0.438 \pm 0.055$ & $0.467 \pm 0.057$ & & \\
\hline Ala-Ala-Ala & $0.081 \pm 0.007$ & $0.089 \pm 0.004$ & $0.101 \pm 0.006$ & $0.109 \pm 0.006$ & $0.125 \pm 0.004$ & & \\
\hline Leu-Gly-Gly & & $0.294 \pm 0.017$ & $0.294 \pm 0.017$ & $0.301 \pm 0.017$ & & $0.305 \pm 0.017$ & \\
\hline Gly-Leu-Gly & & $0.067 \pm 0.003$ & $0.069 \pm 0.003$ & $0.073 \pm 0.003$ & & $0.09 \pm 0.003$ & $0.085 \pm 0.003$ \\
\hline Gly-Gly-Leu & $0.144 \pm 0.011$ & $0.157 \pm 0.011$ & $0.186 \pm 0.012$ & $0.203 \pm 0.012$ & $0.223 \pm 0.013$ & & \\
\hline Pro-Gly-Gly & $0.577 \pm 0.103$ & $0.599 \pm 0.106$ & $0.603 \pm 0.105$ & $0.608 \pm 0.108$ & $0.611 \pm 0.106$ & & \\
\hline Gly-Ala-Leu & & $0.02 \pm 0.001$ & $0.016 \pm 0.001$ & $0.02 \pm 0.001$ & & $0.024 \pm 0.001$ & \\
\hline
\end{tabular}



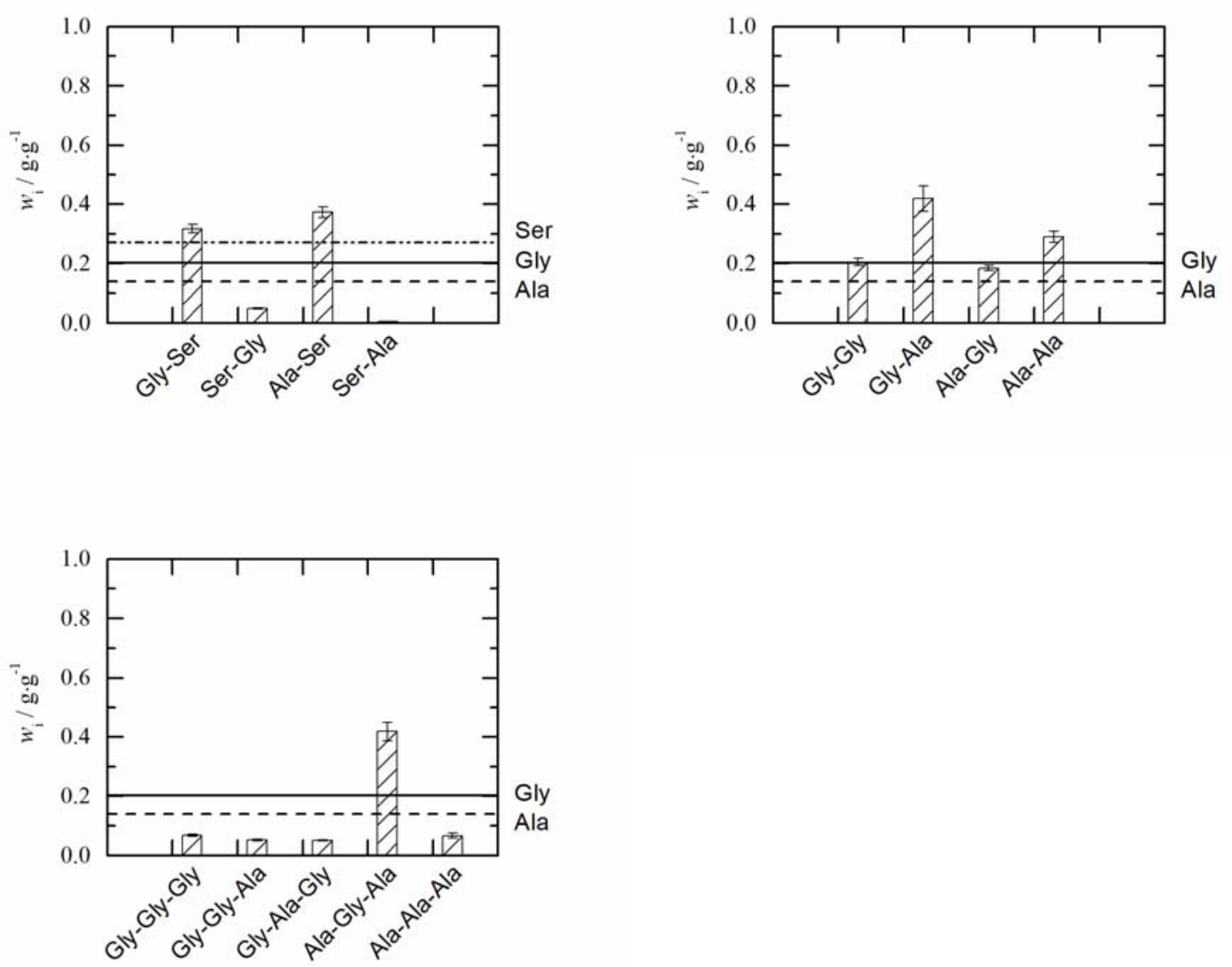

Figure S4 Solubility diagram for the amino acids and peptides at $\mathrm{T}=298.15 \mathrm{~K}$ and $\mathrm{p}=1 \mathrm{~atm}$ shifted to $\mathrm{pH}=7$ from the original data from Table S7 and Table S8 using Henderson Hasselbalch equation (Table S1, S2, Equation S1 and Figure S2 in SI). The single peptides are shown in bars, the amino acids are presented as lines: Gly (solid), Ala (dashed), Leu (dotted), Pro (Dashed-dotted) and Ser (Dashed-double dotted). 
Melting properties

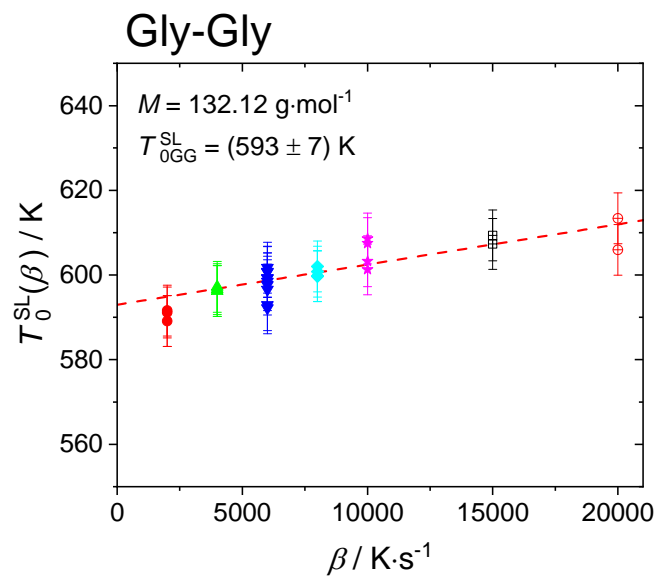

Ala-Gly
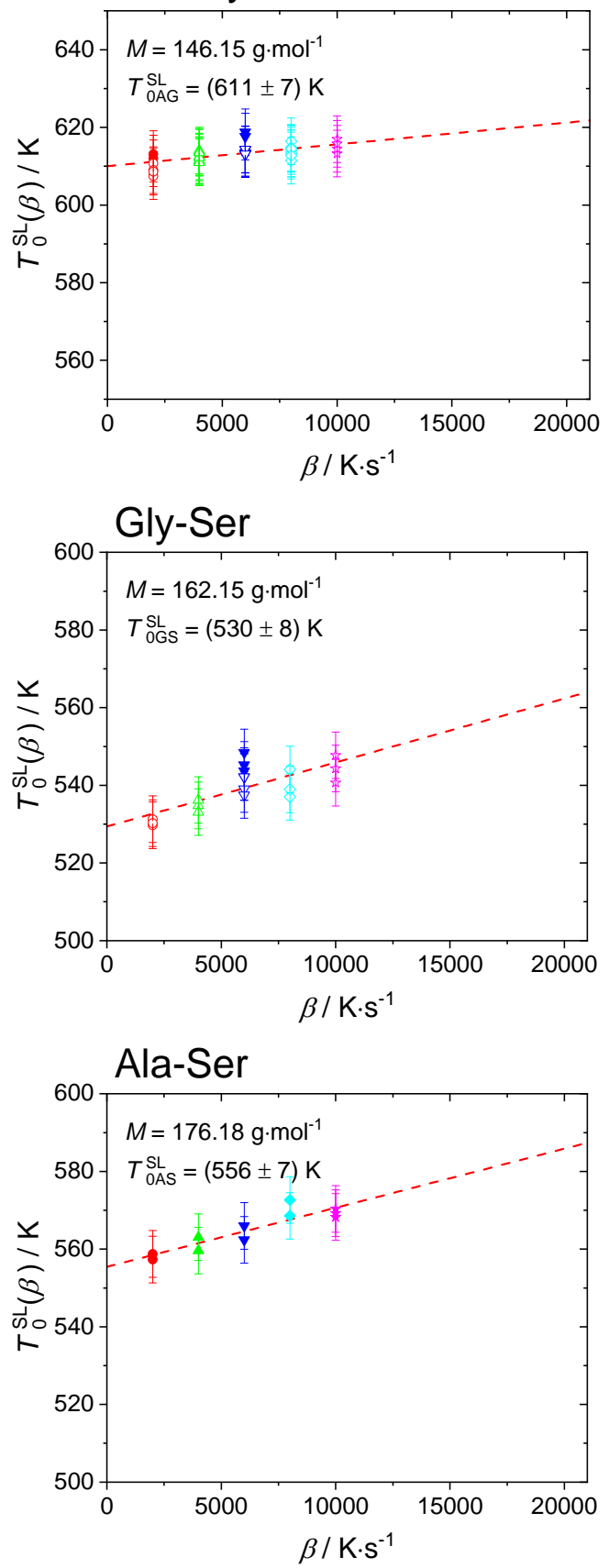

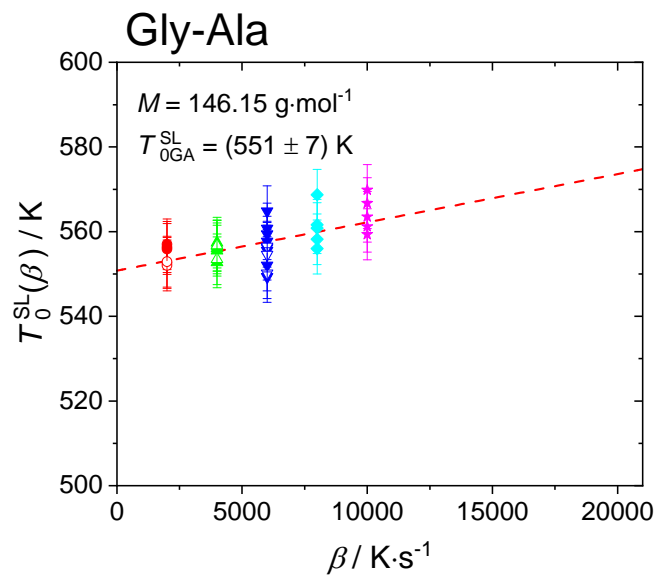

Ala-Ala
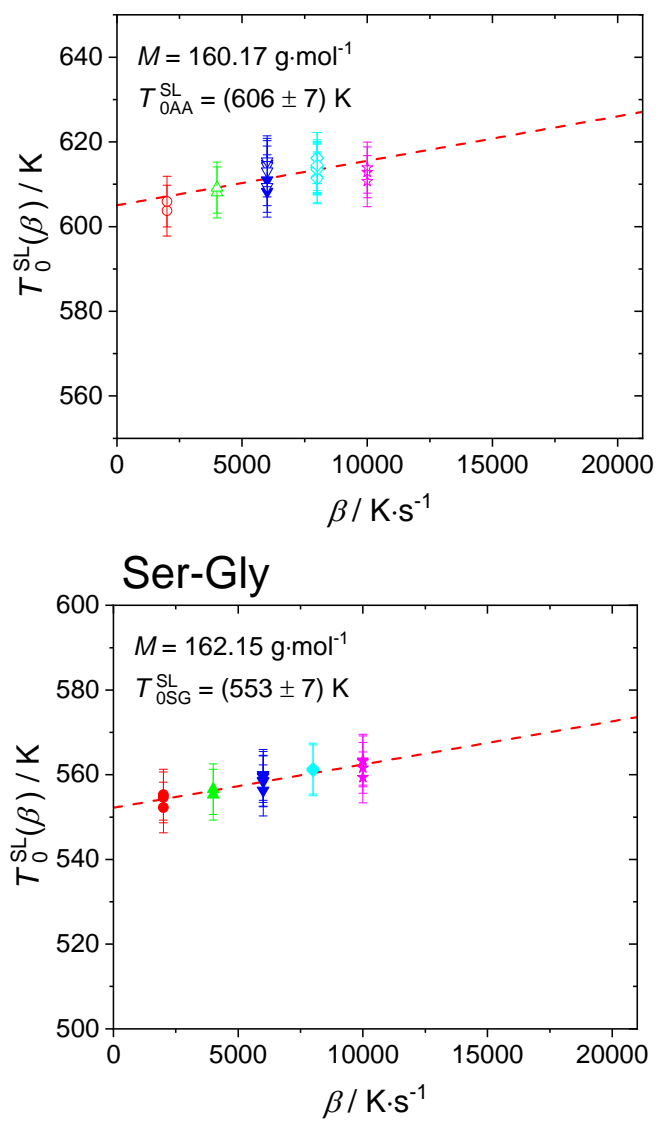

Ser-Ala

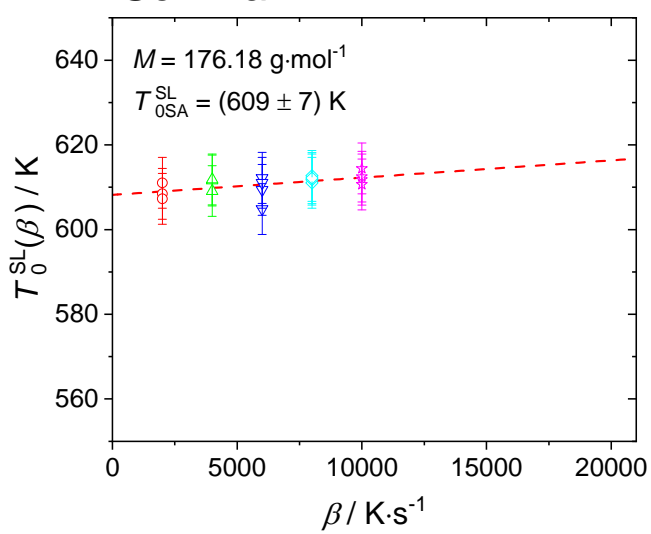



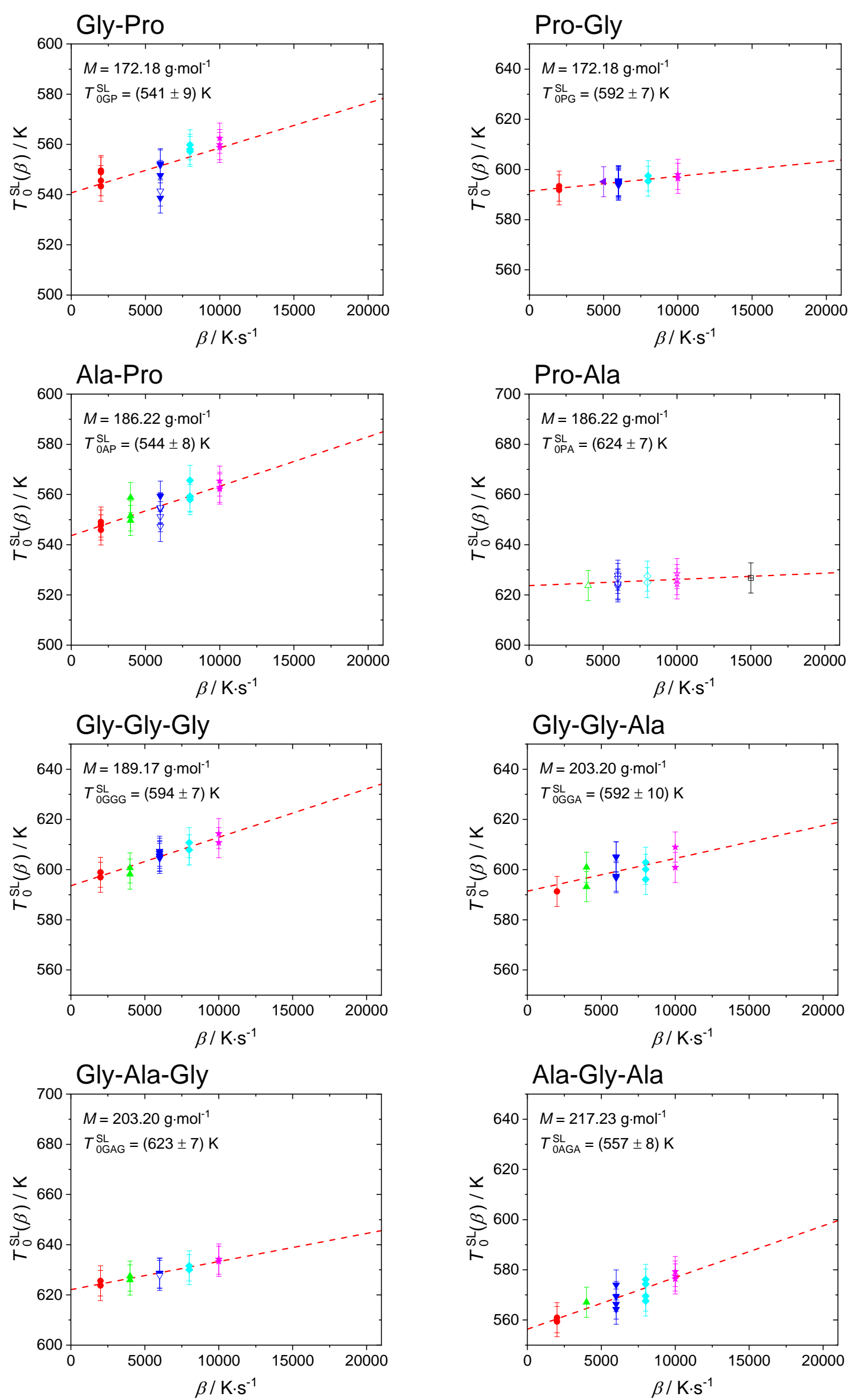

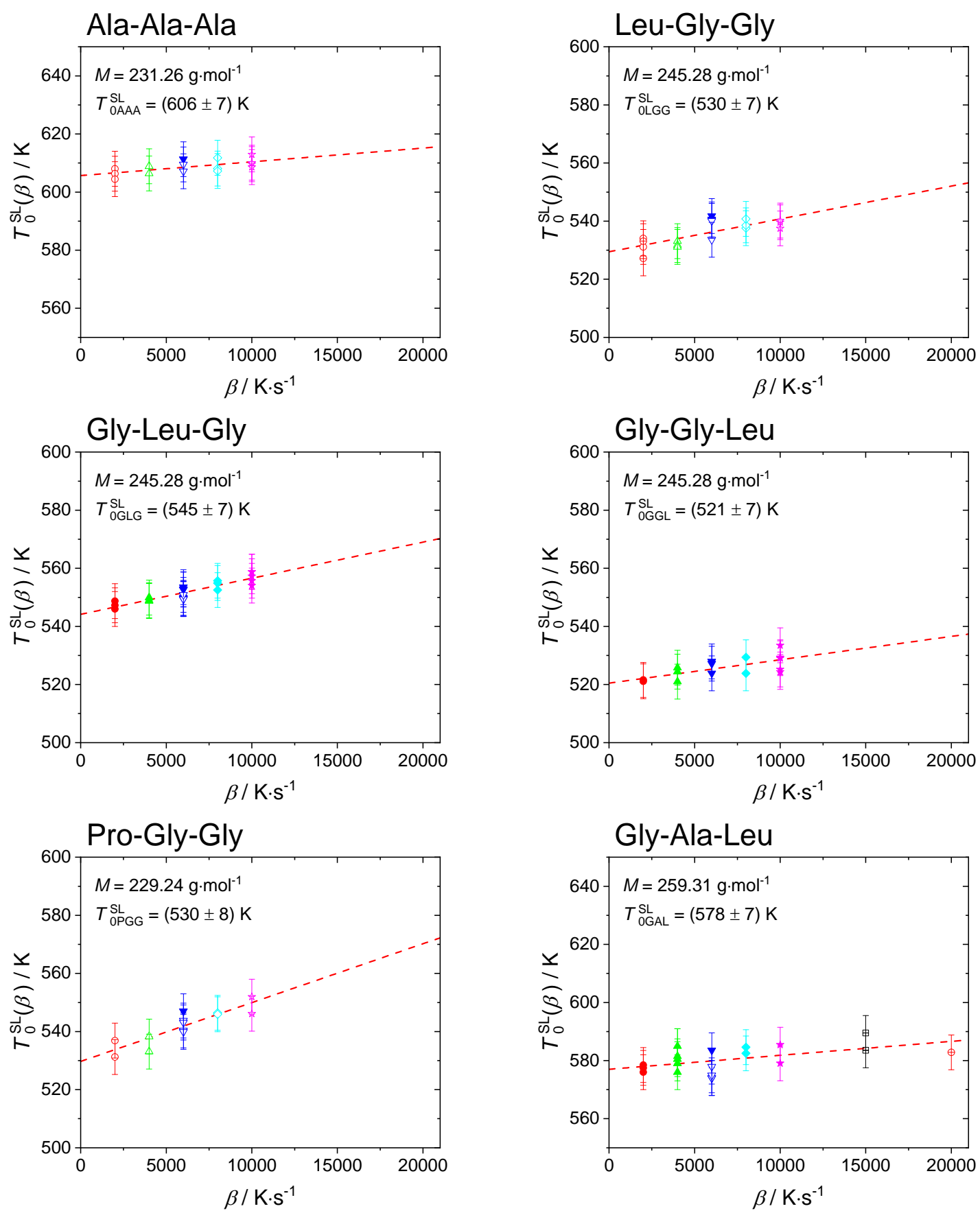

Figure S5. Onset temperature of the melting peak of peptides in heating step \#5, as function of scanning rates and $p=1$ atm. The dashed line denotes linear extrapolation of melting temperatures to zero scanning rates $\Delta T_{0 i}^{S L}(\beta \rightarrow 0)$ The scanning rates used were $1000 \mathrm{Ks}-1$ (black squares), $2000 \mathrm{Ks}-1$ (red circles), $4000 \mathrm{Ks}-1$ (green up-triangles), $5000 \mathrm{Ks}-$ 1 (violet left-triangles), $6000 \mathrm{Ks}-1$ (blue down-triangles), $7000 \mathrm{Ks}-1$ (orange right-triangles), $8000 \mathrm{Ks}-1$ (cyan diamonds), $10000 \mathrm{Ks}-1$ (magenta stars), $15000 \mathrm{Ks}-1$ (black cross squares) and $20000 \mathrm{Ks}-1$ (red cross circles). Solid symbols represent measurements without silicon oil, while empty symbols for measurements with silicon oil. 


\section{Gly-Gly}

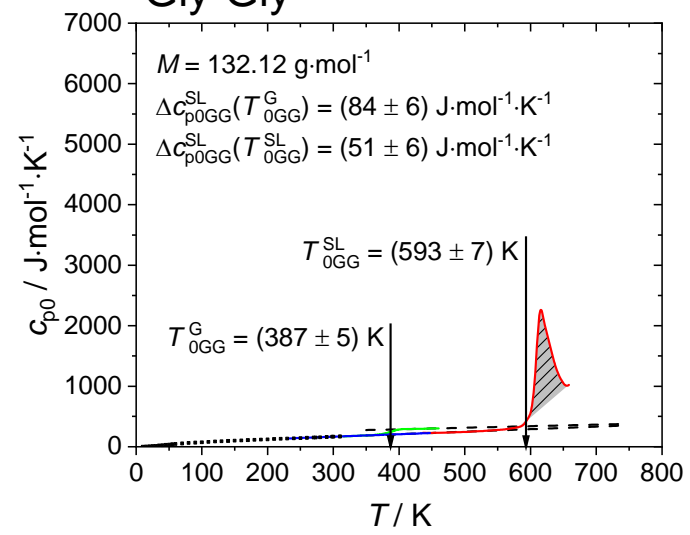

\section{Gly-Ala}
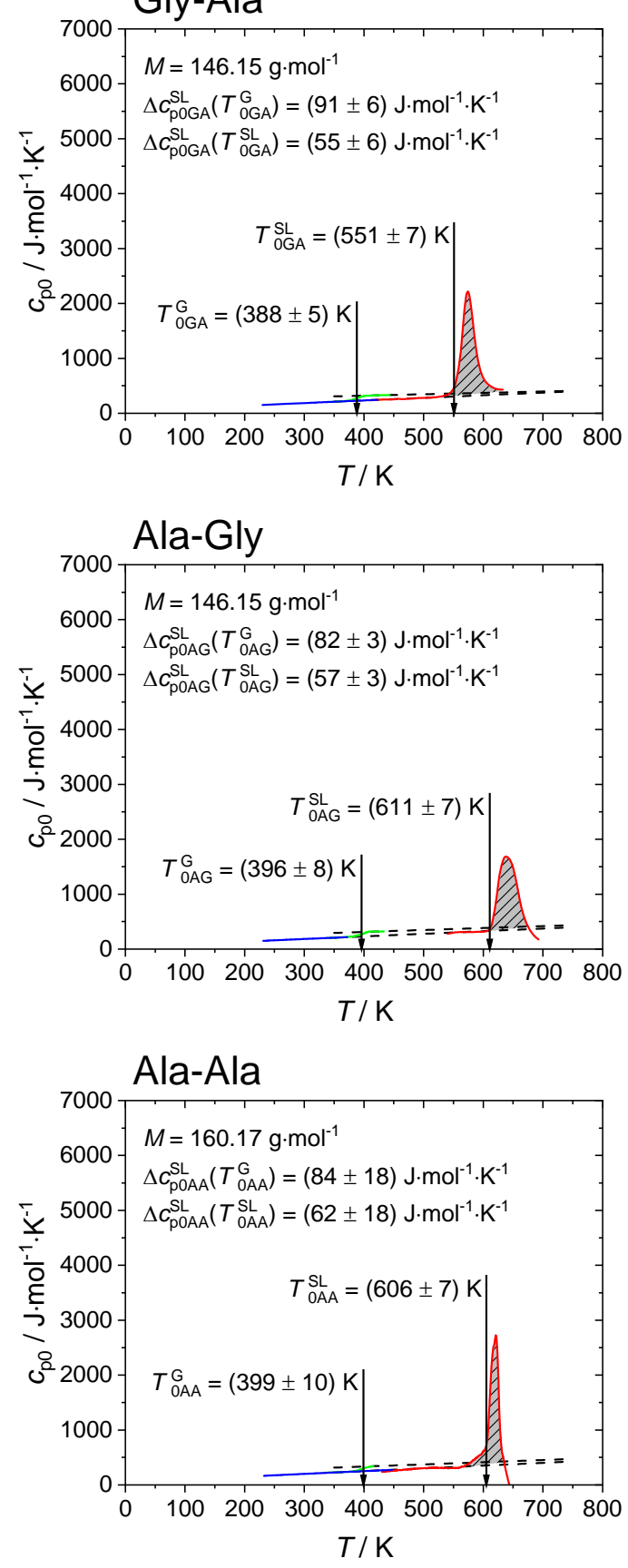
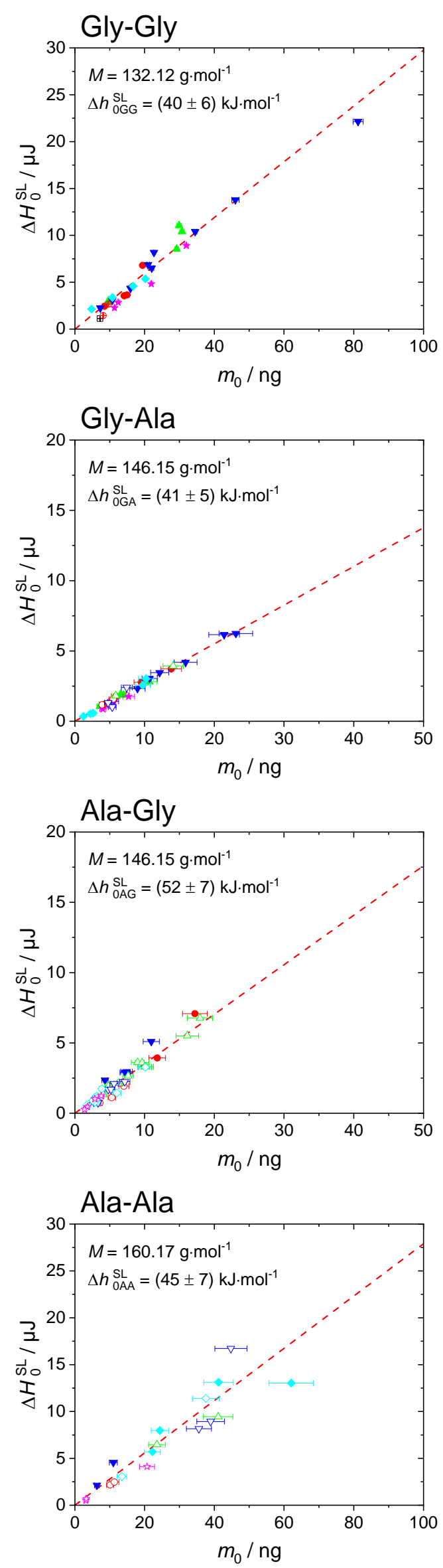

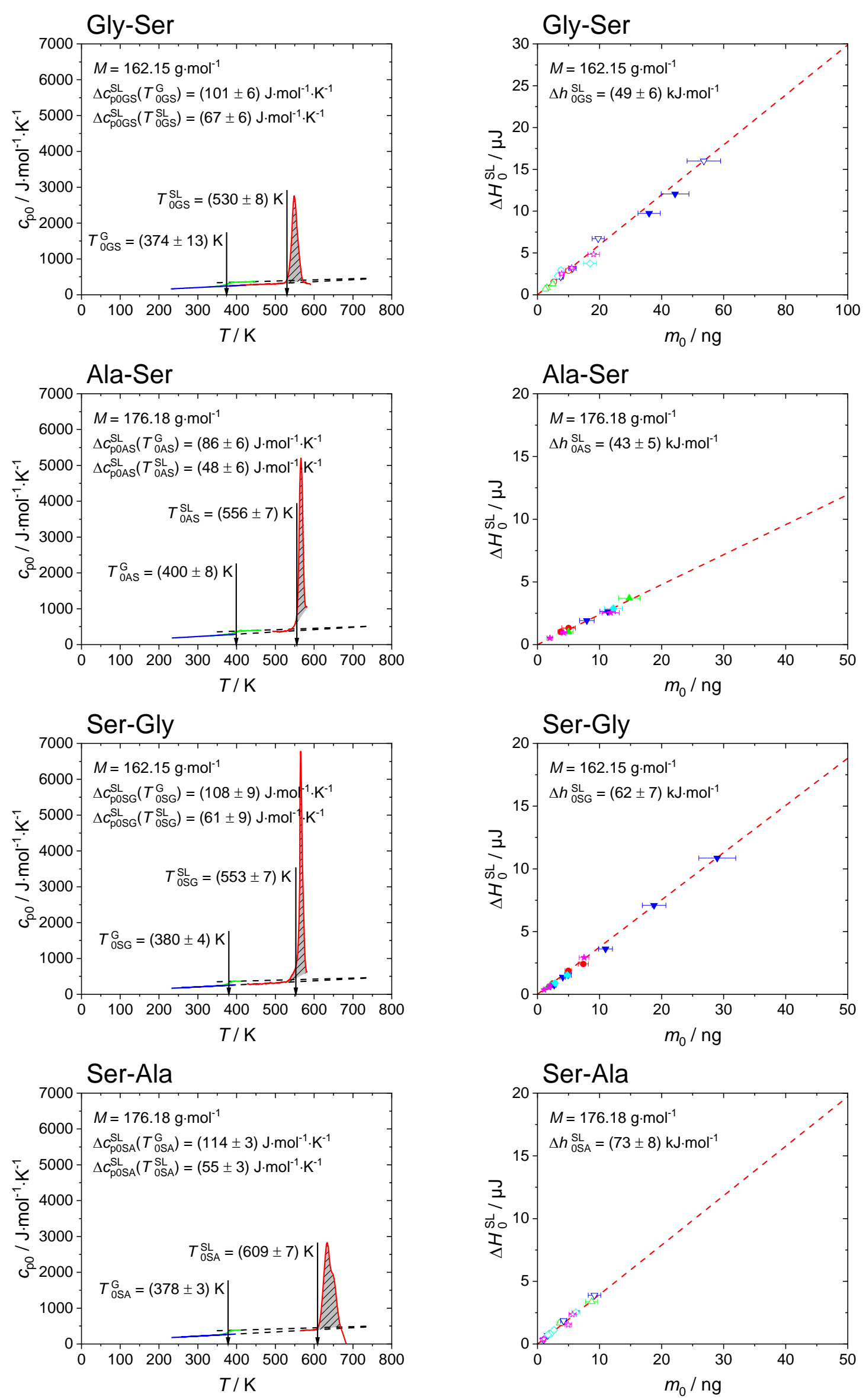


\section{Gly-Pro}
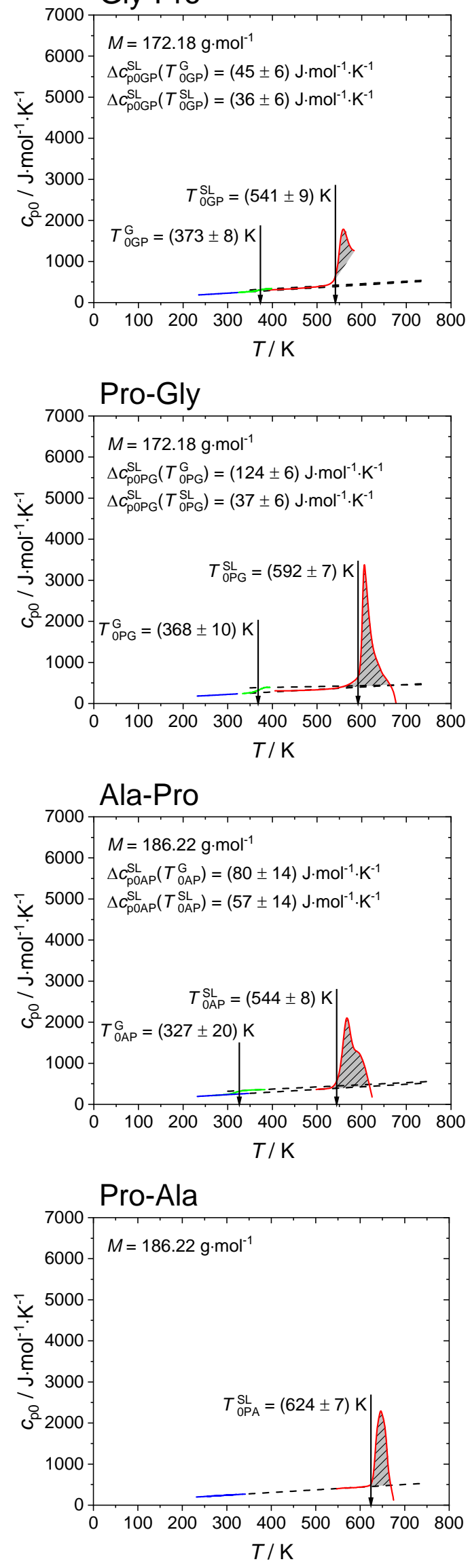
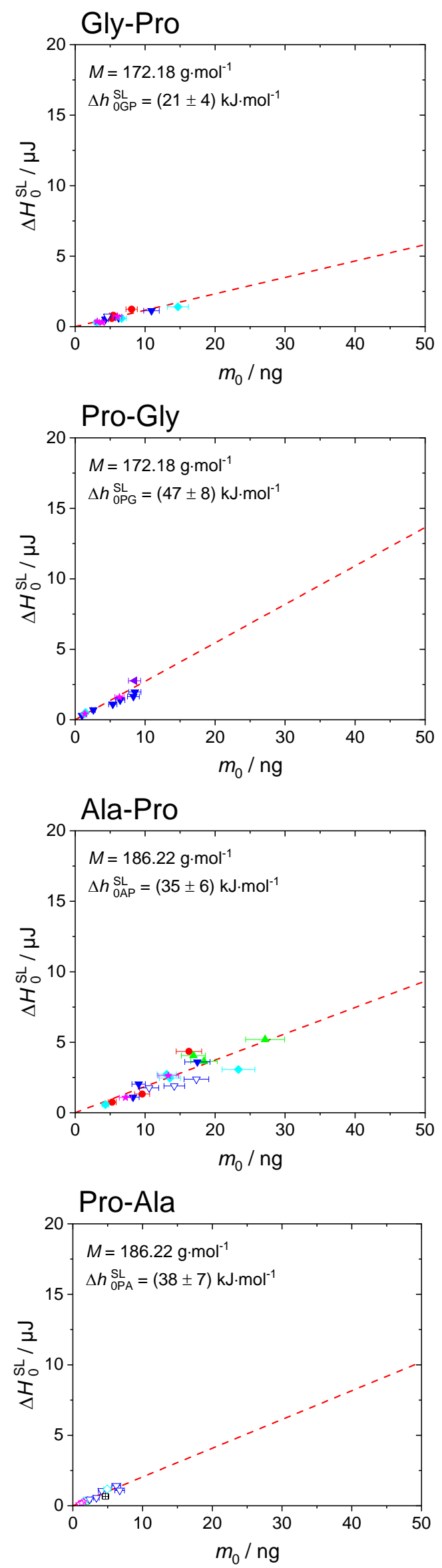

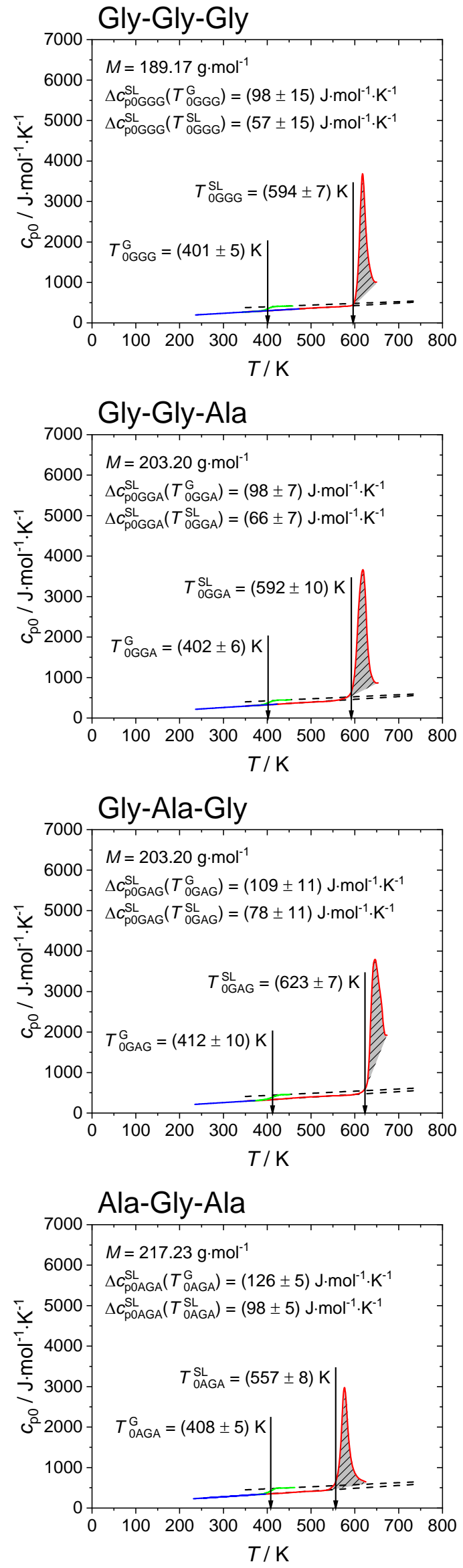
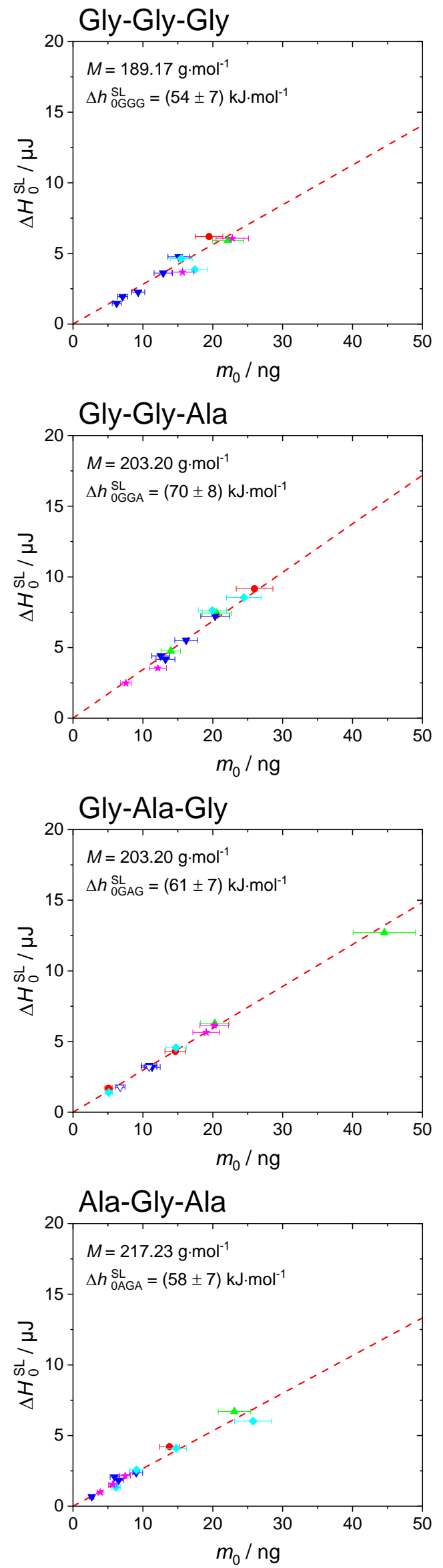

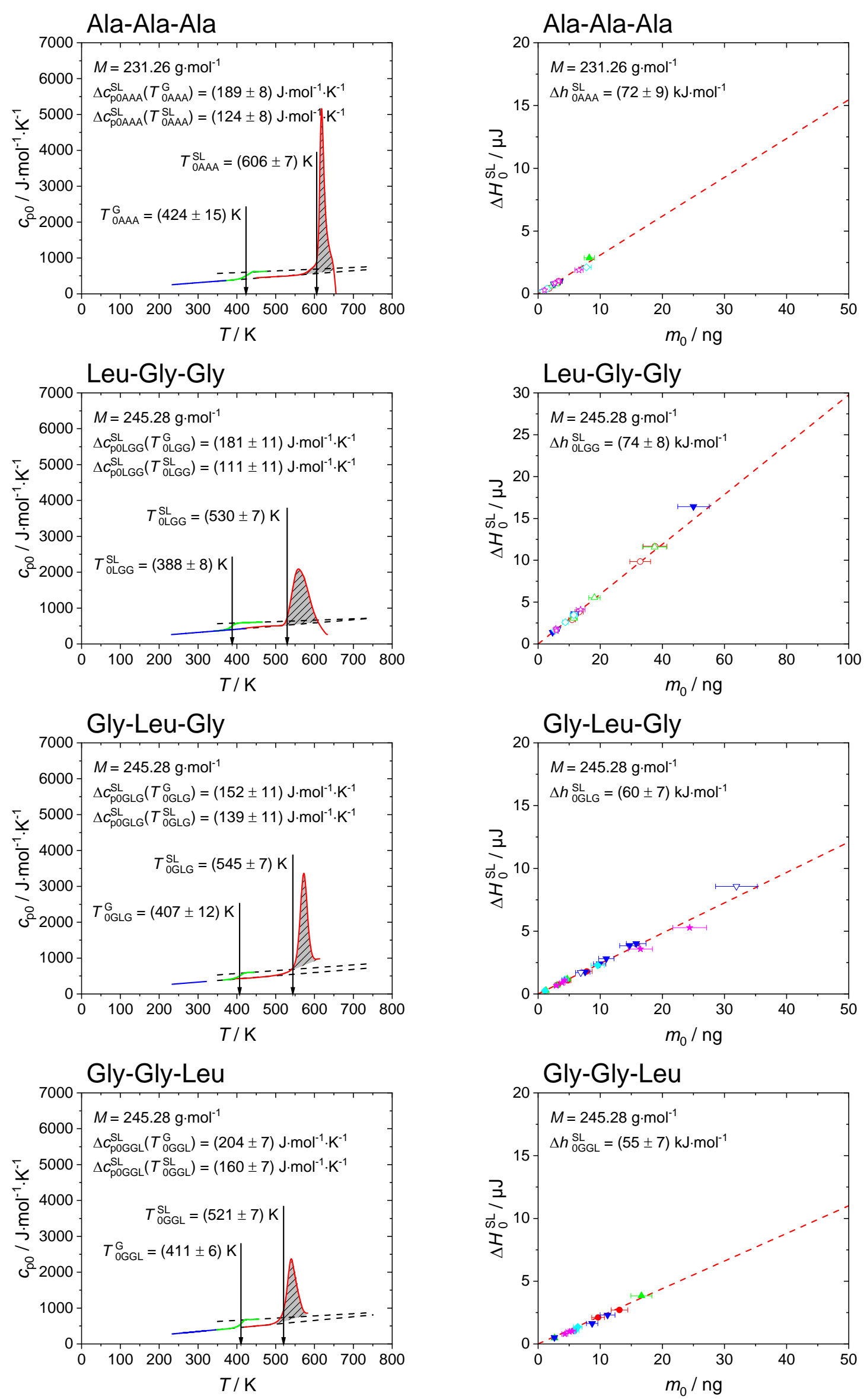

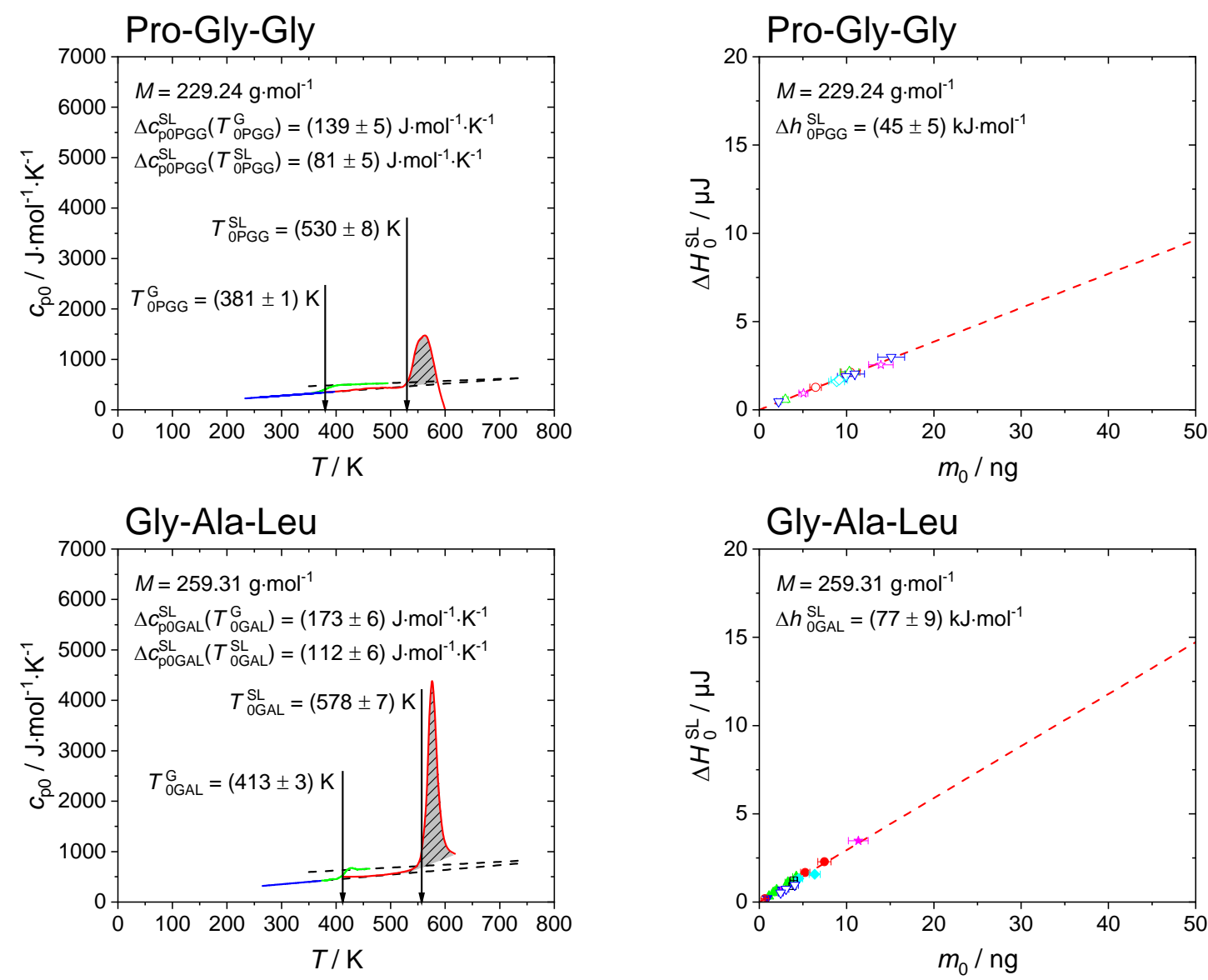

Figure S6 Melting properties of 22 peptides at $\mathrm{p}=1 \mathrm{~atm}$. Left: heat capacity vs. temperature diagrams. Specific heat capacity of peptide. The resulting onset melting temperature by linear extrapolation to zero scanning rates $\Delta T_{0 i}^{S L}(\beta \rightarrow 0)$ of the melting peak of peptide is drawn in the diagram. The heat capacity of solid, $c_{p_{0 i}}{ }^{\text {of }}$ of peptide were measured with standard DSC (solid black lines). The solid red lines represent the glass transition step of ultra-fast quenched melted peptide without silicon oil. The heat capacity of solid $c_{p_{0 i}}^{S}$ and liquid $c_{p_{0 i}}^{L}$ (dashed lines respectively) were linearly fitted to $T_{0 i}^{S L}$. The heat capacity difference between liquid and solid phase were determined at glass transition temperature, $\Delta c_{p_{0 i}}^{S L}\left(T_{0 i}^{G}\right)$ and extrapolation to melting temperature, $\Delta c_{p_{0 i}}^{S L}\left(T_{0 i}^{S L}\right)$. The scanning rates used were $1000 \mathrm{Ks}^{-}$ ${ }^{1}$ (black squares), $2000 \mathrm{Ks}^{-1}$ (red circles), $4000 \mathrm{Ks}^{-1}$ (green up-triangles), $5000 \mathrm{Ks}^{-1}$ (violet left-triangles), $6000 \mathrm{Ks}^{-1}$ (blue down-triangles), $7000 \mathrm{Ks}^{-1}$ (orange right-triangles), $8000 \mathrm{Ks}^{-1}$ (cyan diamonds), $10000 \mathrm{Ks}^{-1}$ (magenta stars), $15000 \mathrm{Ks}^{-}$ ${ }^{1}$ (black cross squares) and $20000 \mathrm{Ks}^{-1}$ (red cross circles). Solid symbols represent measurements without silicon oil, while empty symbols for measurements with silicon oil. The $T_{0 i}^{S L}, \Delta h_{0 i}^{S L}, \Delta c_{p_{0 i}}^{S L}\left(T_{0 i}^{G}\right)$ and $\Delta c_{p_{0 i}}^{S L}\left(T_{0 i}^{S L}\right)$ are listed in Table 2. Right: Enthalpy, $\Delta H_{0 i}^{S L}$, of peptides in respect to sample mass, mo, regardless of the scanning rates. The slope of the linear fit through zero origin (dashed line) signifies the melting enthalpy, $\Delta h_{0 i}^{S L}$. (Right) 


\section{Powder-X-Ray-Diffractograms}
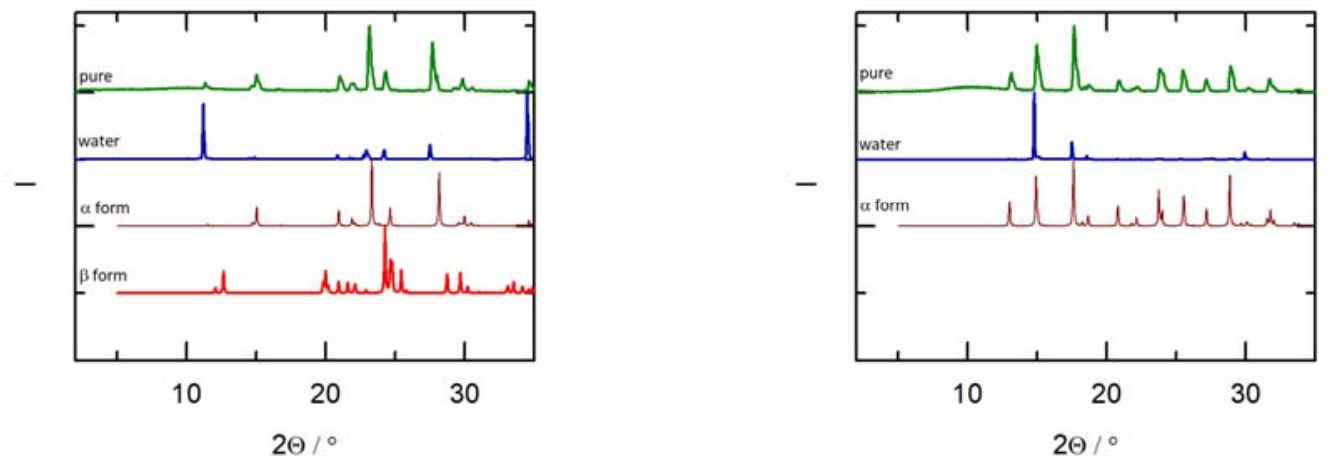

Figure S7 PXRD diffractograms of the peptides: Gly-Gly (left) and Gly-Ala (right). Green line: pure component; black line: solid phase of the supersaturated solution at $\mathrm{T}=298.15 \mathrm{~K}$ and $\mathrm{p}=1 \mathrm{~atm}$.
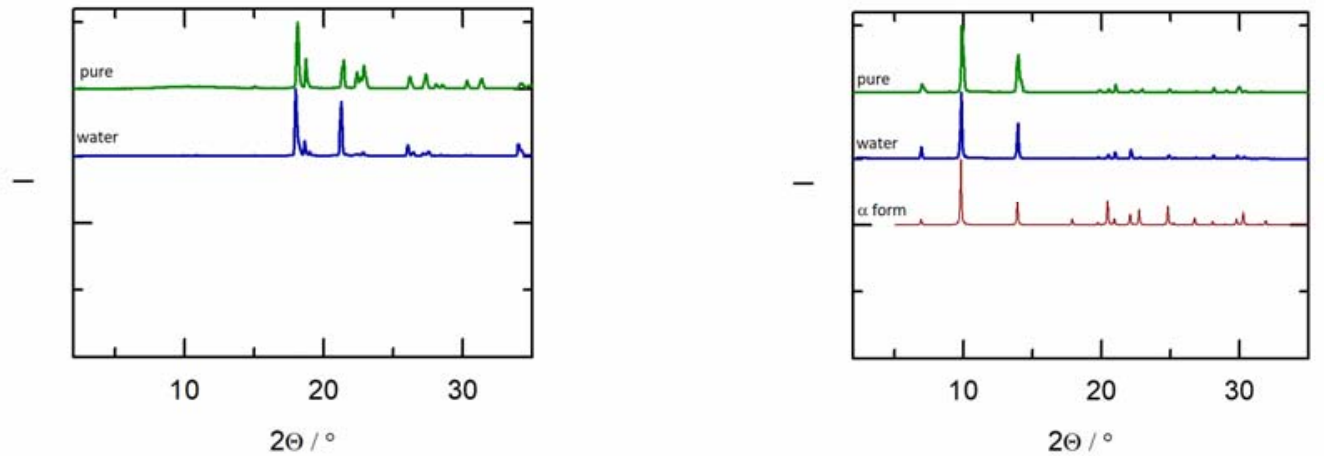

Figure S8 PXRD diffractograms of the peptides: Ala-Gly (left) and Ala-Ala (right). Green line: pure component; black line: solid phase of the supersaturated solution at $\mathrm{T}=298.15 \mathrm{~K}$ and $\mathrm{p}=1 \mathrm{~atm}$.
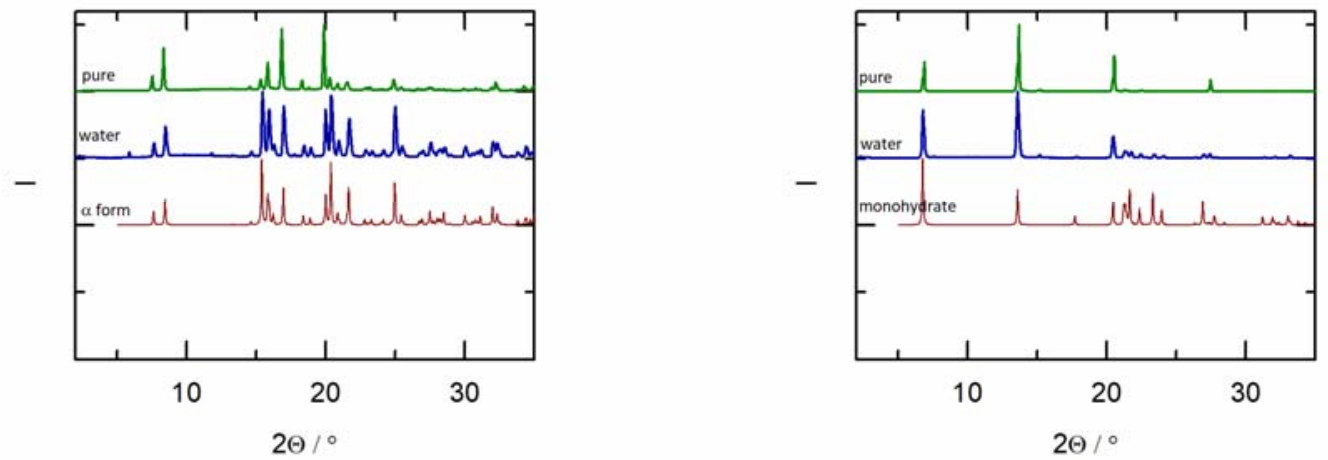

Figure S9 PXRD diffractograms of the peptides: Gly-Pro (left) and Pro-Gly (right). Green line: pure component; black line: solid phase of the supersaturated solution at $\mathrm{T}=298.15 \mathrm{~K}$ and $\mathrm{p}=1 \mathrm{~atm}$. 

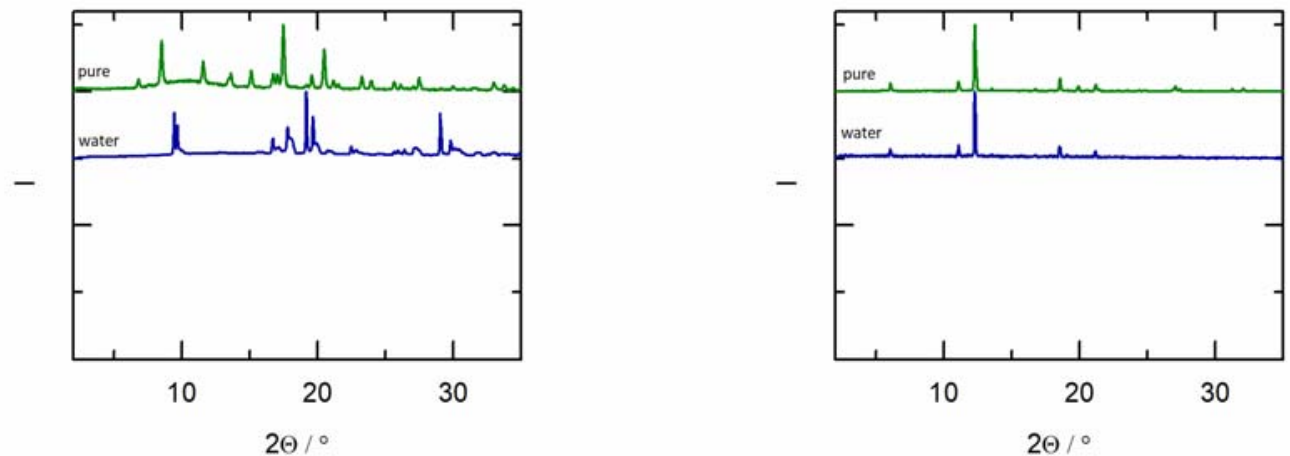

Figure S10 PXRD diffractograms of the peptides: Ala-Pro (left) and Pro-Ala (right). Green line: pure component; black line: solid phase of the supersaturated solution at $\mathrm{T}=298.15 \mathrm{~K}$ and $\mathrm{p}=1 \mathrm{~atm}$.
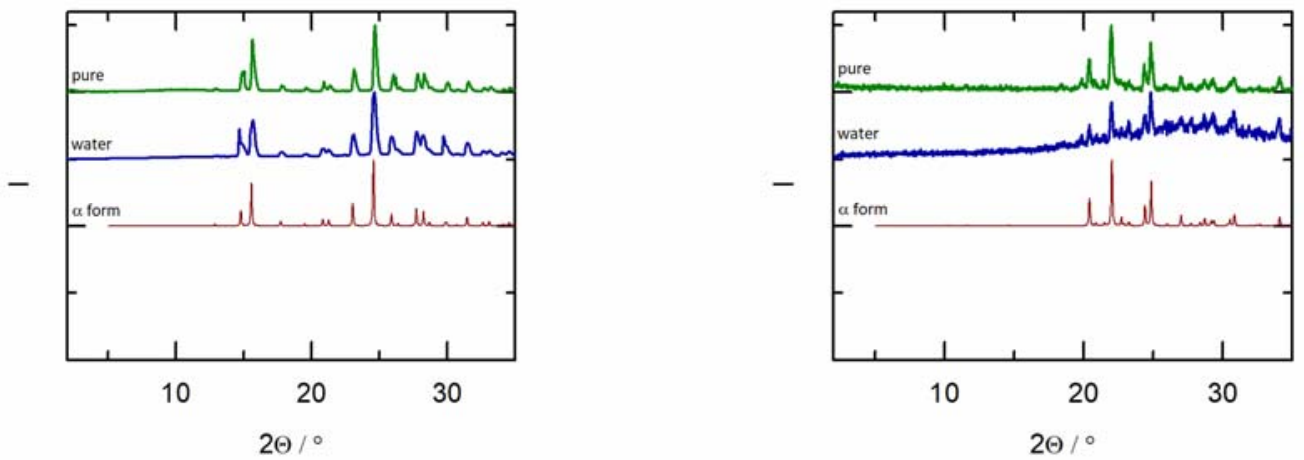

Figure S11 PXRD diffractograms of the peptides: Gly-Ser (left) and Ser-Gly (right). Green line: pure component; black line: solid phase of the supersaturated solution at $\mathrm{T}=298.15 \mathrm{~K}$ and $\mathrm{p}=1 \mathrm{~atm}$.
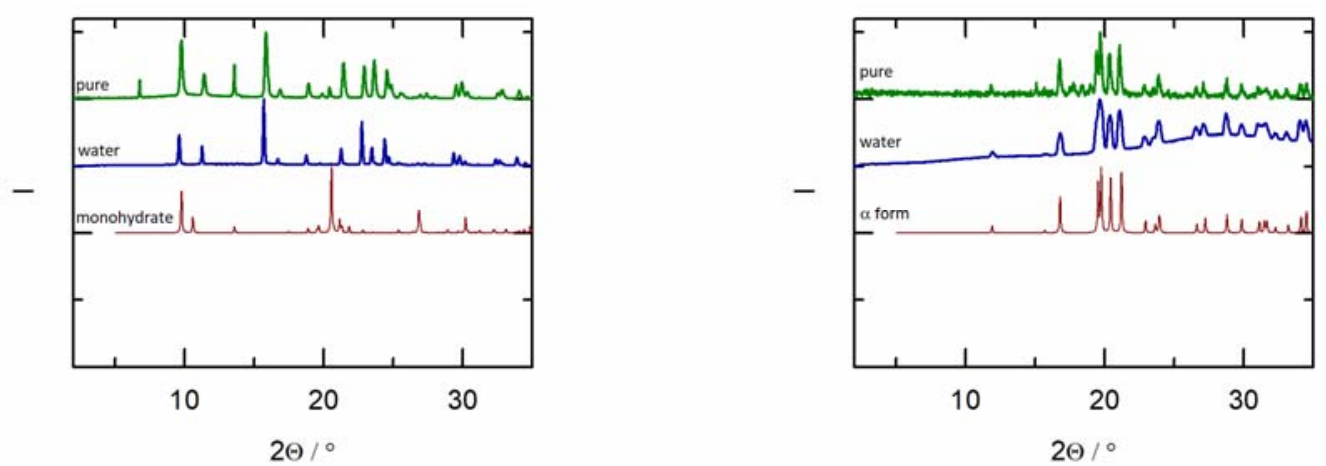

Figure S12 PXRD diffractograms of the peptides: Ala-Ser (left) and Ser-Ala (right). Green line: pure component; black line: solid phase of the supersaturated solution at $\mathrm{T}=298.15 \mathrm{~K}$ and $\mathrm{p}=1 \mathrm{~atm}$. 

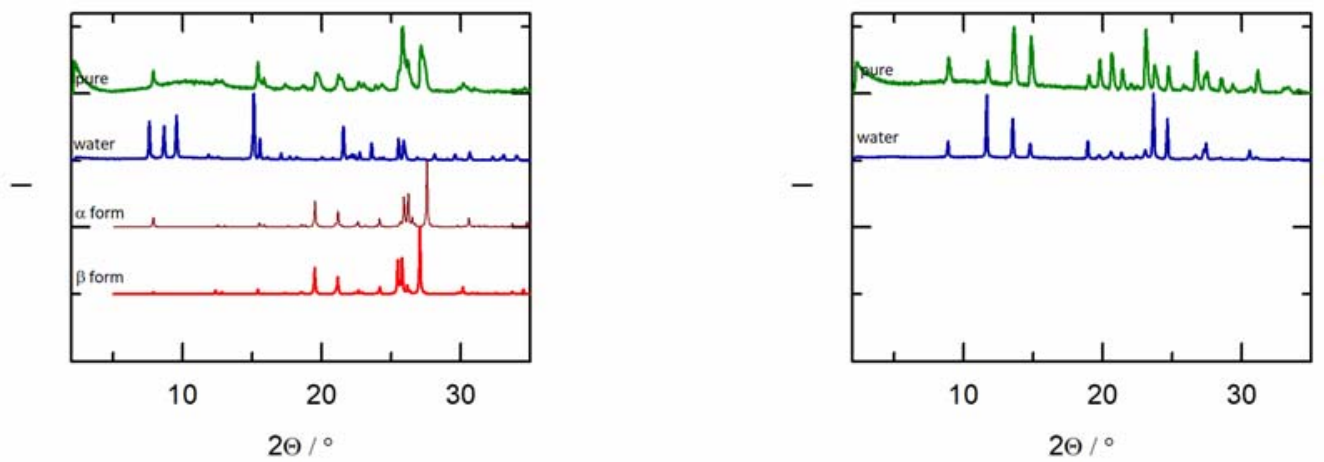

Figure S13 PXRD diffractograms of the peptides: Gly-Gly-Gly (left) and Gly-Gly-Ala (right). Green line: pure component; black line: solid phase of the supersaturated solution at $\mathrm{T}=298.15 \mathrm{~K}$ and $\mathrm{p}=1 \mathrm{~atm}$.
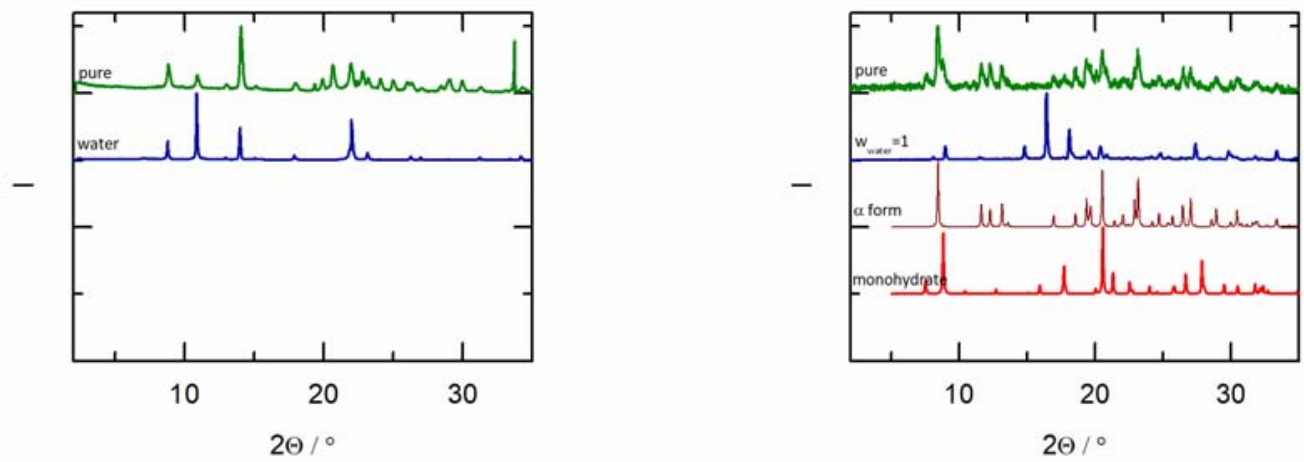

Figure S14 PXRD diffractograms of the peptides: Gly-Ala-Gly (left) and Ala-Gly-Ala (right). Green line: pure component; black line: solid phase of the supersaturated solution at $\mathrm{T}=298.15 \mathrm{~K}$ and $\mathrm{p}=1 \mathrm{~atm}$.
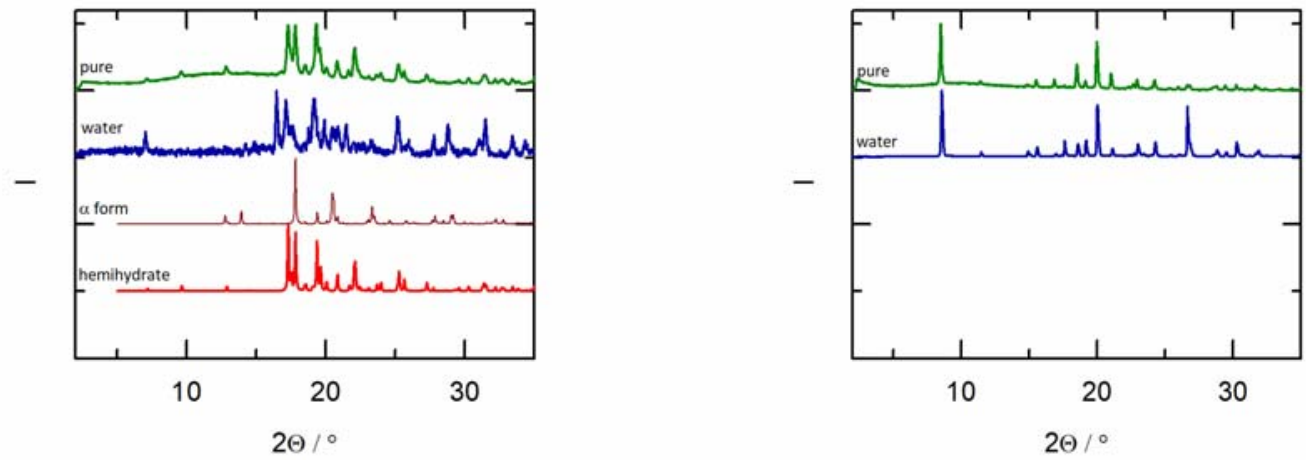

Figure S15 PXRD diffractograms of the peptides: Ala-Ala-Ala (left) and Leu-Gly-Gly (right). Green line: pure component; black line: solid phase of the supersaturated solution at $\mathrm{T}=298.15 \mathrm{~K}$ and $\mathrm{p}=1 \mathrm{~atm}$. 

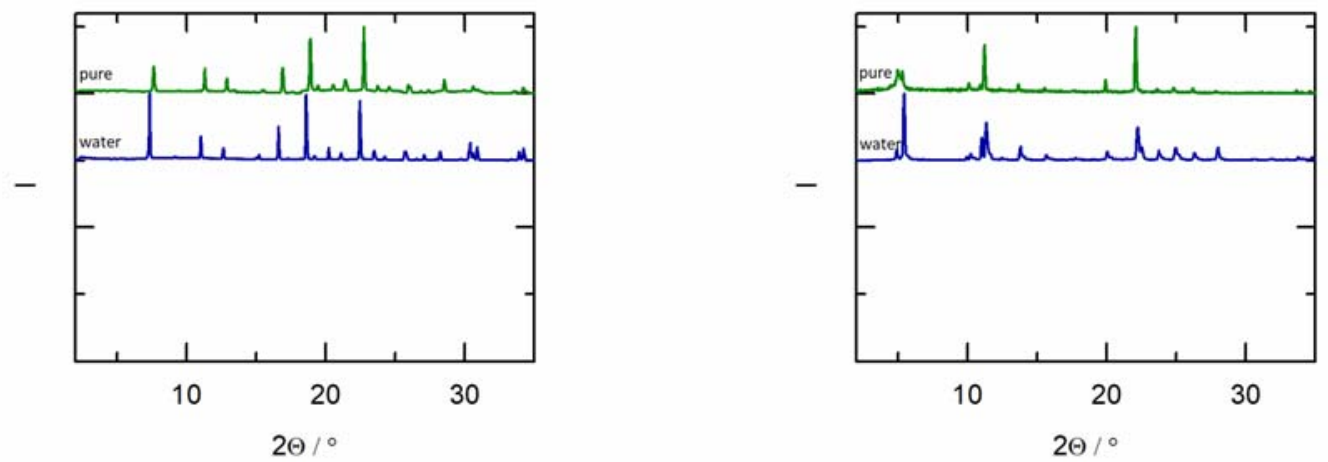

Figure S16 PXRD diffractograms of the peptides: Gly-Leu-Gly (left) and Gly-Leu-Leu (right). Green line: pure component; black line: solid phase of the supersaturated solution at $\mathrm{T}=298.15 \mathrm{~K}$ and $\mathrm{p}=1 \mathrm{~atm}$.
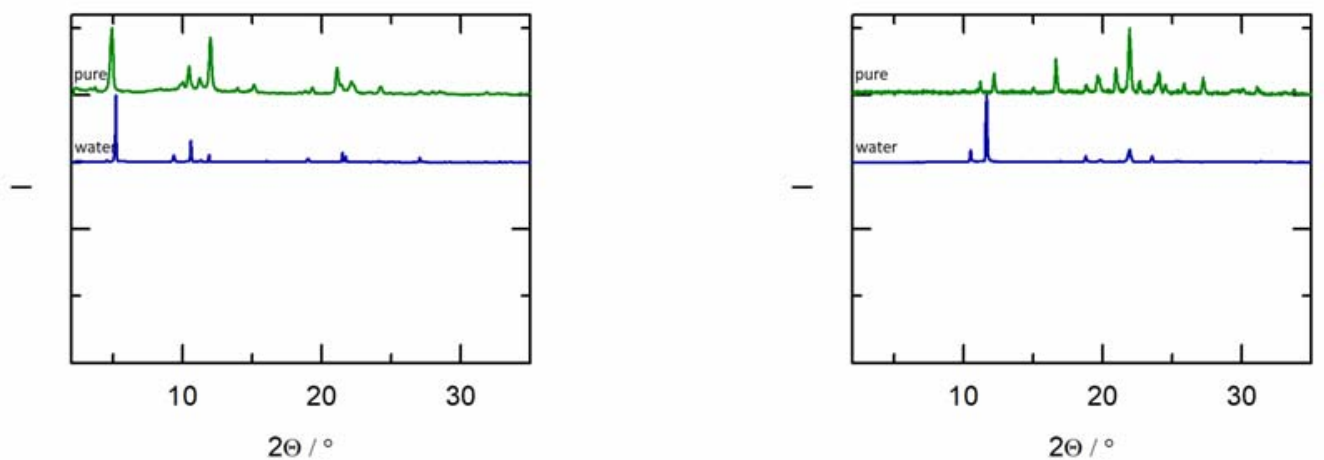

Figure S17 PXRD diffractograms of the peptides: Gly-Ala-Leu (left) and Pro-Gly-Gly (right). Green line: pure component; black line: solid phase of the supersaturated solution at $\mathrm{T}=298.15 \mathrm{~K}$ and $\mathrm{p}=1 \mathrm{~atm}$. 

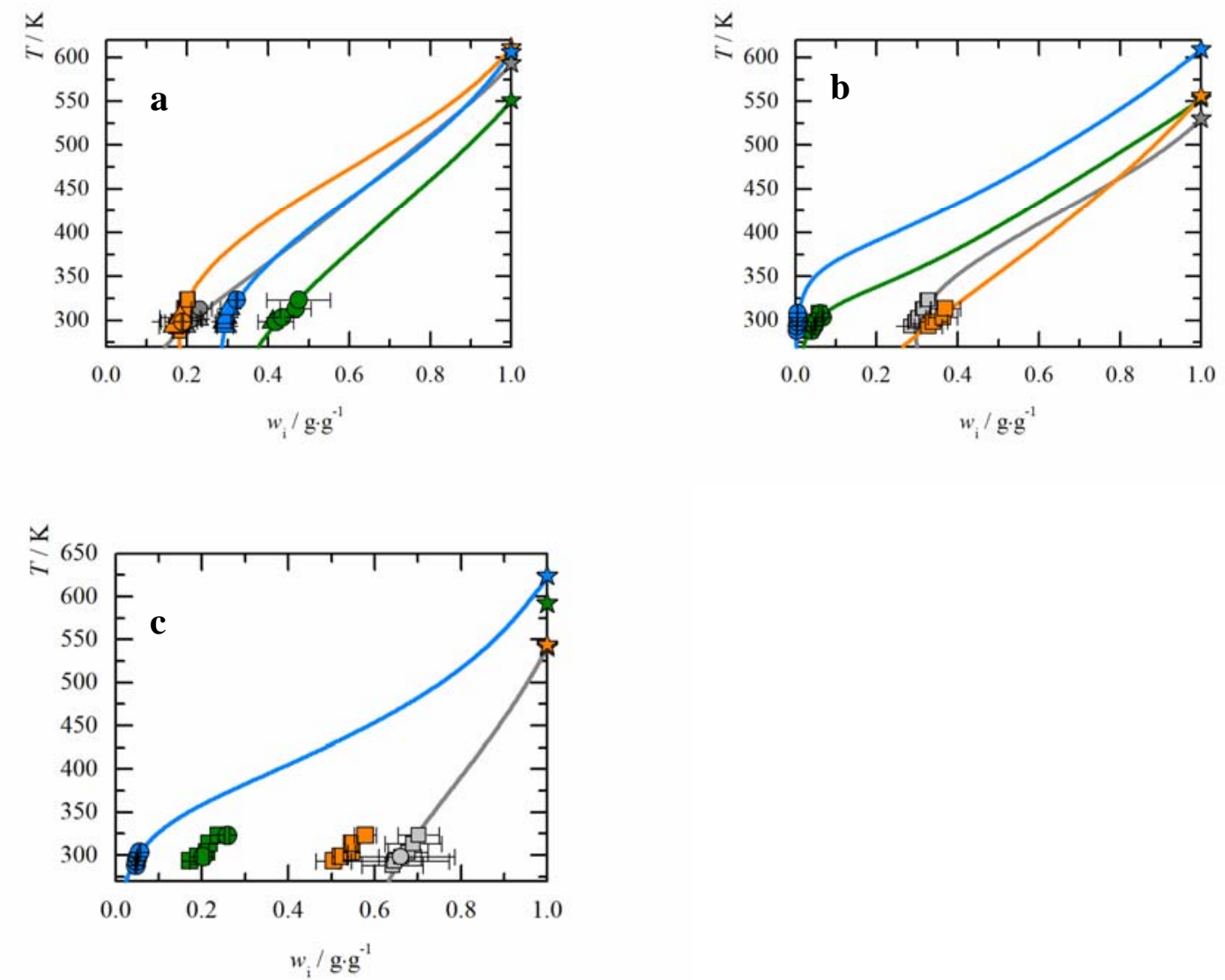

Figure S18 Solubility diagrams of dipeptides in temperature vs. weight fraction at $p=1 \mathrm{~atm}$. Solid symbols represent the experimental determined solubility: squares (photometric method) and circles (gravimetric method). Solid stars represent the melting temperatures determined with FSC in this work. Solid lines represent the PC-SAFT modeled solubility using the parameters from Table 4 and the melting properties from Table 3 and adjusted to $\mathrm{pH}=7 \mathrm{using}$ Henderson Hassel-balch. a: Dipeptides based on Gly and Ala: Gly-Ala (green), Ala-Ala (blue), Gly-Gly (grey), AlaGly (orange) and. b: Dipeptides based on Gly, Ala and Ser: Ala-Ser (orange), Gly-Ser (grey), Ser-Gly (green), and SerAla (blue). c: Dipeptides based on Gly, Ala and Pro: Gly-Pro (grey), Ala-Pro (orange), Pro-Gly (green) and Pro-Ala (blue).

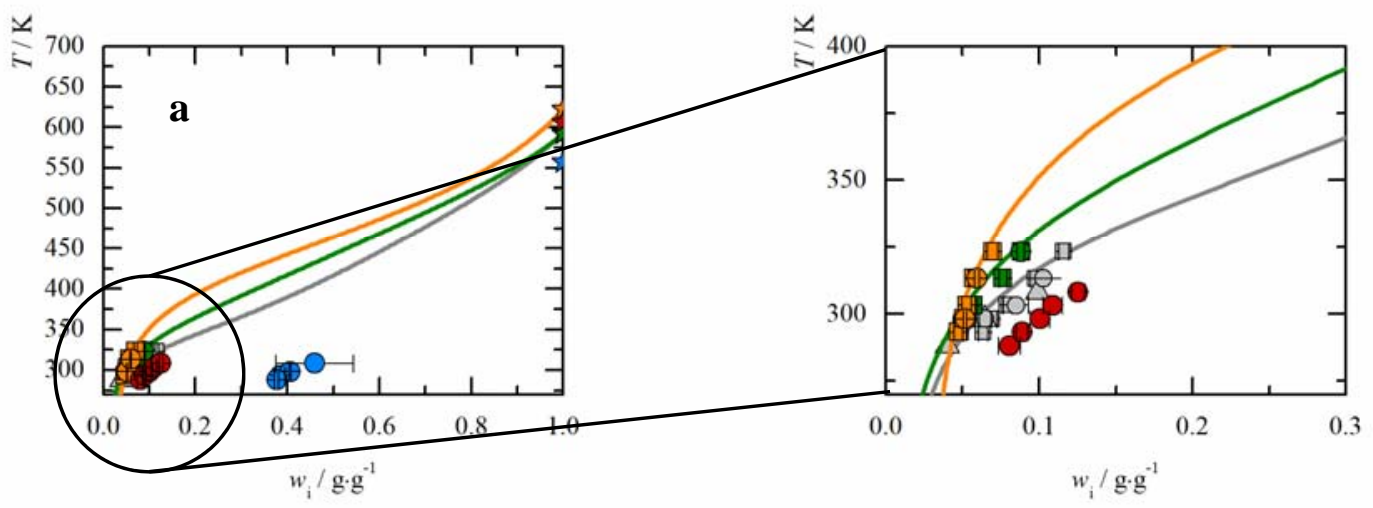




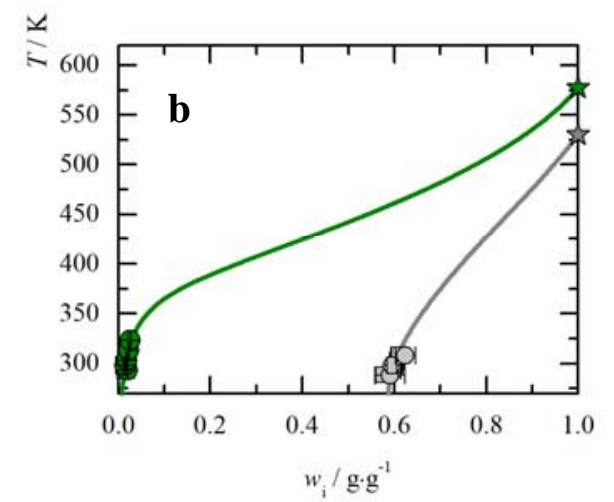

Figure S19 Solubility diagrams of tripeptides in temperature vs. weight fraction at $\mathrm{p}=1 \mathrm{~atm}$. Solid symbols represent the experimental determined solubility: squares (photometric method) and circles (gravimetric method). Solid stars represent the melting temperatures determined with FSC in this work. Solid lines represent the PC-SAFT modeled solubility using the parameters from Table 4 and the melting properties from Table 3 and adjusted to $\mathrm{pH}=7 \mathrm{using}$ Henderson Hassel-balch.. a: Tripeptides based on Gly and Ala: Gly-Ala-Gly (orange), Gly-Gly-Ala (green), Gly-GlyGly (grey), Ala-Ala-Ala (red) and Ala-Gly-Ala (blue). b: Tripeptides based on Gly, Ala, Leu and Pro: Pro-Gly-Gly (grey) and Gly-Ala-Leu (green).

\section{References}

1. V. Mathot, M. Pyda, T. Pijpers, G. Vanden Poel, E. van de Kerkhof, S. van Herwaarden, F. van Herwaarden and A. Leenaers, Thermochimica Acta, 2011, 522, 36.

2. S. van Herwaarden, E. lervolino, F. van Herwaarden, T. Wijffels, A. Leenaers and V. Mathot, Thermochimica Acta, 2011, 522, 46.

3. Y. Z. Chua, H. T. Do, C. Schick, D. Zaitsau and C. Held, RSC Adv., 2018, 8, 6365.

4. H. T. Do, Y. Z. Chua, J. Habicht, M. Klinksiek, M. Hallermann, D. Zaitsau, C. Schick and C. Held, RSC Adv., 2019, 9, 32722.

5. A. Abdelaziz, D. H. Zaitsau, N. V. Kuratieva, S. P. Verevkin and C. Schick, Phys. Chem. Chem. Phys. PCCP, 2019, 12787.

6. A. Abdelaziz, D. H. Zaitsau, T. A. Mukhametzyanov, B. N. Solomonov, P. Cebe, S. P. Verevkin and C. Schick, Thermochim. Acta, 2017, 657, 47.

7. P. Cebe, X. Hu, D. L. Kaplan, E. Zhuravlev, A. Wurm, D. Arbeiter and C. Schick, Sci. Rep., 2013, 3, 1130 .

8. P. Cebe, D. Thomas, J. Merfeld, B. P. Partlow, D. L. Kaplan, R. G. Alamo, A. Wurm, E. Zhuravlev and C. Schick, Polym., 2017, 126, 240.

9. Y. Z. Chua, H. T. Do, C. Schick, D. H. Zaitsau and C. Held, RSC Adv., 2018, 8, 6365.

10. H. T. Do, Y. Z. Chua, J. Habicht, M. Klinksiek, M. Hallermann, D. H. Zaitsau, C. Schick and C. Held, RSC Adv., 2019, 9, 32722.

11. A. Avdeef, Advanced drug delivery reviews, 2007, 59, 568.

12. J. Gross and G. Sadowski, Ind. Eng. Chem. Res., 2001, 40, 1244.

13. J. M. Prausnitz, E. G. de Azevedo and R. N. Lichtenthaler, Molecular thermodynamics of fluidphase equilibria, Prentice Hall PTR, Upper Saddle River, N.J, 1999.

14. Jeffrey P. Wolbach and Stanley I. Sandler, INd. Eng. Chem., 1998, 2917. 